\title{
INTEGRATED CONTROL OF NEXT GENERATION POWER SYSTEM
}

\author{
Final Report
}

\author{
US DoE Award No. DE-FC26-06NT42793
}

October 1, 2006 to February 28, 2010

\section{Project Investigator}

\author{
Advanced Power and Electricity Research Center (APERC) \\ Lane Department of Computer Science and Electrical Engineering (LD CSEE) \\ West Virginia University (WVU) Morgantown, WV \\ Project Partners \\ National Energy Technology Laboratory (NETL) \\ Southern California Edison (SCE)
}

\section{Project Overview}

The multi-agent system (MAS) approach has been applied with promising results for enhancing an electric power distribution circuit, such as the Circuit of the Future as developed by Southern California Edison. These next generation power system results include better ability to reconfigure the circuit as well as the increased capability to improve the protection and enhance the reliability of the circuit.

There were four main tasks in this project. The specific results for each of these four tasks and their related topics are presented in main sections of this report. Also, there were seven deliverables for this project. The main conclusions for these deliverables are summarized in the identified subtask section of this report. The specific details for each of these deliverables are included in the "Project Deliverables" section at the end of this Final Report.

This report was prepared as an account of work sponsored by an agency of the United States Government. Neither the United States Government nor any agency thereof, nor any of their employees, makes any warranty, express or implied, or assumes any legal liability or responsibility for the accuracy, completeness, or usefulness of any information, apparatus, product, or process disclosed, or represents that its use would not infringe privately owned rights. Reference herein to any specific commercial product, process, or service by trade name, trademark, manufacturer, or otherwise does not necessarily constitute or imply its endorsement, recommendation, or favoring by the United States Government or any agency thereof. The views and opinions of authors expressed herein do not necessarily state or reflect those of the United States Government or any agency thereof. 


\section{TABLE OF CONTENTS}

Title Page $\quad \ldots \ldots \ldots \ldots \ldots \ldots \ldots \ldots \ldots \ldots \ldots \ldots \ldots \ldots \ldots \ldots$

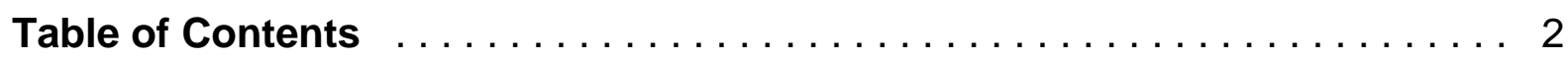

Executive Summary $\ldots \ldots \ldots \ldots \ldots \ldots \ldots \ldots \ldots \ldots \ldots \ldots \ldots \ldots \ldots \ldots \ldots$

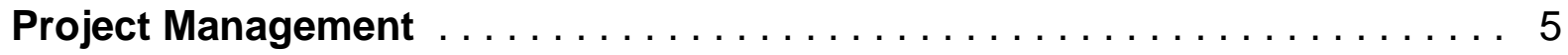

Project Team $\ldots \ldots \ldots \ldots \ldots \ldots \ldots \ldots \ldots \ldots \ldots \ldots \ldots \ldots \ldots \ldots \ldots \ldots \ldots \ldots \ldots$

\section{Project Tasks}

Task 1. Review of Existing Technologies and Technical Outcomes. . . . . . . 8

1.0 Introduction.

1.1 Project Relationship with SCE CoF Project.

1.2 Compile List of Equipment Deployed and Planned for SCE CoF Grid.

1.3 Study Deployed and Planned Equipment to be Integrated into SCE CoF Grid.

1.4 Establish Requirements for Equipment to be Implemented in SCE CoF Grid.

Task 2. Multi-Agent Systems.

2.0 Introduction.

2.1 Adapt Unidirectional Control Agents to Multidirectional AC Power Systems.

2.2 Evaluate Agent Technologies for Managing Real-World SCE CoF Grid Scenarios.

2.3 Assess Ability of Agents to Interface and Interact with Grid Devices.

2.4 Develop CoF Distribution System Modeled using Graph Theory.

2.5 Integrate MAS Technologies into a Prototype Laboratory System for Testing.

2.6 Demonstrate Multi-Agent Concepts on a Laboratory Testbed of a Power System.

Task 3. Modeling and Simulation.

3.0 Introduction.

3.1 Ascertain from Literature Review if Similar Research Projects Offer Collaboration.

3.2. Study Feeders Typical to CoF for Attributes and Agents to Assimilate in Project.

3.3 Select through Consults with Utilities a Program to Analyze the SCE CoF Grid.

3.4 Adapt NETL Capabilities to Enhance the Current CoF Capabilities.

Task 4. Economic Analysis and Evaluation.

4.0 Introduction.

4.1 Evaluate Broad-Based Economic Analysis of Incorporating MAS in CoF Grid.

4.2 Assess Benefit Cost Analysis of Deploying MAS in SCE CoF Grid.

4.3 Develop Case Studies using Utility Input to Estimate Benefits of MAS on CoF.

4.4 Document SCE Support for Economic Feasibility and Benefit Analysis through Asset Planning. 
Deliverable 1. Report on Technical Requirements for a Multi-Agent System.

Deliverable 2. Reports on Economic Evaluation and Economic Feasibility.

Deliverable 3. Catalog of Current Technologies for Power Systems.

Deliverable 4. Report on Challenges for Sensor Communications and Controls.

Deliverable 5. White Paper on General Concepts of a Multi-Agent System.

Deliverable 6. Report on Specific Applications in Modeling and Simulation.

Deliverable 7. Reports on Simulation Studies and Laboratory Tests of a Multi-Agent System.

Main Project Accomplishments $\ldots \ldots \ldots \ldots \ldots \ldots \ldots \ldots \ldots \ldots$

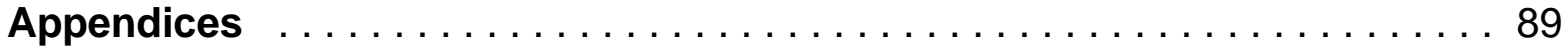

Appendix 1. Project Fact Sheet.

Appendix 2. Project Publications.

Appendix 3. Bibliography \& Cited References. 


\section{EXECUTIVE SUMMARY}

Control methodologies provide the necessary data acquisition, analysis and corrective actions needed to maintain the state of an electric power system within acceptable operating limits. These methods are primarily software-based algorithms that are nonfunctional unless properly integrated with system data and the appropriate control devices. Components of the control of power systems today include protective relays, supervisory control and data acquisition (SCADA), distribution automation (DA), feeder automation, software agents, sensors, control devices and communications. Necessary corrective actions are still accomplished using large electromechanical devices such as vacuum, oil and gas-insulated breakers, capacitor banks, regulators, transformer tap changers, reclosers, generators, and more recently FACTS (flexible AC transmission system) devices.

The recent evolution of multi-agent system (MAS) technologies has been reviewed and effort made to integrate MAS into next generation power systems. A MAS can be defined as “...a loosely-coupled network of problem solvers that work together to solve problems that are beyond their individual capabilities...”. These problem solvers, often called agents, are autonomous and may be heterogeneous in nature. This project has shown that a MAS has significant advantages over a single, monolithic, centralized problem solver for next generation power systems.

Various communication media are being used in the electric power system today, including copper, optical fiber and power line carrier (PLC) as well as wireless technologies. These technologies have enabled the deployment of substation automation (SA) at many facilities. Recently, carrier and wireless technologies have been developed and demonstrated on a pilot basis. Hence, efforts have been made by this project to penetrate these communication technologies as an infrastructure for next generation power systems.

This project has thus pursued efforts to use specific MAS methods as well as pertinent communications protocols to imbed and assess such technologies in a real electric power distribution system, specifically the Circuit of the Future (CoF) developed by Southern California Edison (SCE).

By modeling the behavior and communication for the components of a MAS, the operation and control of the power distribution circuit have been enhanced. The use of MAS to model and integrate a power distribution circuit offers a significantly different approach to the design of next generation power systems. For example, ways to control a power distribution circuit that includes a micro-grid while considering the impacts of thermal constraints, and integrating voltage control and renewable energy sources on the main power system have been pursued. Both computer simulations and laboratory testbeds have been used to demonstrate such technologies in electric power distribution systems.

An economic assessment of MAS in electric power systems was also performed during this project. A report on the economic feasibility of MAS for electric power systems was prepared, and particularly discusses the feasibility of incorporating MAS in transmission and distribution (T\&D) systems. Also, the commercial viability of deploying MAS in T\&D systems has been assessed by developing an initial case study using utility input to estimate the benefits of deploying MAS. 
In summary, the MAS approach, which had previously been investigated with good success by APERC for naval shipboard applications, has now been applied with promising results for enhancing an electric power distribution circuit, such as the Circuit of the Future developed by Southern California Edison. The results for next generation power systems include better ability to reconfigure circuits, improve protection and enhance reliability.

\section{PROJECT MANAGEMENT}

\section{Research Team}

The team for this research project consisted of the Advanced Power \& Electricity Research Center (APERC) at West Virginia University (WVU), the Office of Research and Development (ORD) at the USDoE National Energy Technology Laboratory (NETL) and the Southern California Edison (SCE) utility in California. The WVU/APERC effort on the project was led by Ali Feliachi, who assembled in November 2006 a group of faculty members and graduate research assistants. The USDoE/ORD/NETL effort on the project was originally led by Bill Rogers, who was subsequently replaced by Paul Hines in January 2007. Initially, in November 2006, Charles Vartanian was the lead person for the SCE effort. Charlie was later joined by Stephanie Hamilton.

\section{Project Coordination}

Project coordination was accomplished through discussions via phone calls, emails and project team meetings. One such meeting was held at SCE on July 31, 2007 with Paul Hines on site and APERC participating via conference call. The agenda for the meeting included a presentation on agent technology and the SCE Circuit of the Future (CoF), as well as an update on planned equipment and possible scenarios of operation for the CoF.

At the start of the project APERC made appointment offers to fill administrative and research staff positions. However, hiring policies at WVU were often a challenge to this effort. In addition, personnel changes at both NETL and SCE hindered the effectiveness of both of these team members' participation after the first year of the project.

\section{Management Schedule}

Project progress was managed through weekly meetings and special seminars and annual meetings of the APERC Advisory Committee held at WVU. In the early phases of the project, (as discussed in the third quarterly progress report) a schedule (with titles for tasks/descriptions) and timelines (with milestones/deliverables/status) was developed and plotted for the remainder of the project.

\section{Published Reports}

The research team prepared and submitted 13 Quarterly Progress Reports for the project to the USDoE/NETL. These reports are available in both .pdf and .doc formats from APERC. Reports were also prepared and presented at Program/Peer Review meetings. This Final Report is a condensation of these reports, particularly the Quarterly Reports. 


\section{PROJECT TEAM}

\section{Project Investigators}

West Virginia University (WVU) Morgantown, WV

Advanced Power and Electricity Research Center (APERC)

College of Mineral Resources and Engineering (CEMR)

Lane Department of Computer Science and Electrical Engineering (LD CSEE)

Ali Feliachi, Principal Investigator

Muhammad Choudhry

Afzel Noore

Daryl Reynolds

Sophia Wan

Karl Schoder

Department of Mechanical and Aerospace Engineering

John E. (Ed) Sneckenberger

Eberly College of Arts and Sciences

Department of Mathematics

Hong-Jian Lai

Davis College of Design, Environment and Resources

Division of Resources

John Saymansky

Project Partners

National Energy Technology Laboratory (NETL)

Bill Rogers

Paul Hines

Southern California Edison (SCE)

Stephanie Hamilton

Charlie Vartanian

Project Manager

Mario Sciulli, NETL, US Department of Energy

This report is the result of efforts by individuals and organizations who collaborated with APERC/WVU on this project. The contributions of the individuals and organizations listed above are greatly appreciated. In addition, the advice and support of the U.S. Department of Energy Office of Electricity Delivery and Energy Reliability, and Mario Sciulli of the National Energy Technology Laboratory Project Management Center are gratefully acknowledged. 


\section{Researcher Expertise and Perspective}

Each of the eight researchers from APERC provided an expertise to the project, as well as a perspective on agent based technologies. The Table below lists these expertise and perspectives.

$\begin{array}{ccc}\text { Researcher } & \text { Project Expertise } & \text { Agent Perspective } \\ \text { Ali Feliachi } & \text { Multiple Agents } & \text { Advanced Agents } \\ \text { Muhammad Choudhry } & \text { Laboratory Simulator } & \text { Applied Agents } \\ \text { Afzel Noore } & \text { Agent Clustering } & \text { Fewer Agents } \\ \text { Daryl Reynolds } & \text { System Communications } & \text { Faster Agents } \\ \text { Sophia Wan } & \text { System Protection } & \text { Smarter Agents } \\ \text { Hong-Jian Lai } & \text { System Analyses } & \text { Better Agents } \\ \text { John Saymansky } & \text { System Economics } & \text { Economical Agents } \\ \text { John E. Sneckenberger } & \text { Project Summary } & \text { Documented Agents }\end{array}$

\section{Jointly Performed Research}

There were 13 subtasks that were to be jointly performed by APERC in partnership with SCE and/or NETL (1.1, 1.2, 1.3, 1.4, 2.7, 3.1, 3.2, 3.3, 3.5, 3.6, 4.3, 4.4 and 4.5). These subtasks involved partnering on equipment specifications for existing distribution circuits in the electric power grid, planning the hardware design and details for envisioned circuits of the current system, and proposing a new scheme for the vision of a better grid in the future.

\section{Pertinent Project Publications}

The members of the project team were productive publishers of the results of the research conducted during the project. There were a total of 18 articles published in research journals as well as in professional proceedings for conferences and symposiums. A list of project related publications is included in Appendix 2. These publications include the journal paper by A. Karimi, S. Eftekharnejad and A. Feliachi titled "Reinforcement Learning Based Backstepping Control of Power System Oscillations" that was published in the Electric Power Systems Research Journal, Vol. 79, pp. 1511-1520, 2009.

During the period of the project, five masters degree students completed their theses and one doctoral degree student completed his dissertation. This doctoral student was A. Karimi and the title of his dissertation was "Power System Damping Controllers Design using a Backstepping Control Technique”. He earned his doctorate degree from the WVU Lane Department of Computer Science and Electrical Engineering in August 2008. 


\section{Task 1. \\ Review of Existing Technologies and Technical Outcomes.}

\subsection{Introduction.}

The performance of existing technologies for power systems will be reviewed and the desired technical outcomes for a multi-agent system (MAS) will be proposed.

\subsubsection{Subtasks for Task 1 .}

This task consisted of four subtasks as follows:

1.1. Perform a literature search and compile a list of distributed generation (DG), distributed energy resources (DER), and demand side management (DSM) presently deployed by Southern California Edison, along with the protection, control, communication and interconnection devices typically associated with such installations. In support of this subtask, SCE will i) provide a tabulation and summary of the utility system performance characteristics and/or conditions for which a device interconnecting to the SCE CoF Grid may need to meet a particular technical criteria, ii) compile a table of existing devices and control systems on the SCE T\&D grid that could be considered 'intelligent' and identify the existing and advanced technologies that SCE plans to deploy on the CoF, and iii) provide a summary of the typical circuit/system occurrences on the SCE system, noting the types of events for which SCE would record or log related information.

1.2. Catalog/categorize the types of DG/DER/DSM planned for deployment on the CoF during development of the smart agents proposed for this project, along with the related protection, control, communication and interconnection devices.

1.3. Study strengths and weaknesses of the current sensing, protection and communication systems, and identify any impediments to integration of the proposed MAS, and

1.4. Establish performance requirements for evaluation of the multi-agent system capability to assess transmission and distribution (T\&D) system status, adjust to typical system occurrences and coordinate response actions in harmony with adjacent devices, including self-configuration upon loss of one or more agents. The smart agents shall demonstrate ability to learn and retain information for reference or for use during a future event.

\subsubsection{Deliverables for Task 1.}

Four deliverables were expected for Task 1. The first, Deliverable 1, is a report on the Technical Requirements for a Multi-Agent System. Deliverable 2 is a topical report on the economic feasibility of increasing T\&D level intelligence. Note that Deliverable 2 is included as part of the report for Task 4. The third deliverable, Deliverable 3, is a catalog of the type/level of intelligence presently available in various electricity delivery and DG system communications and control devices. And finally, Deliverable 4 is a report on the Challenges for Sensor Communications and Controls. 


\subsection{Project Relationship with SCE CoF Project.}

Nationally, the generation, transmission and distribution of electric power continues to experience increased load demand, added environmental constraints and revised market strategies. In addition, electrical power systems are operated by utility companies who have been reluctant to undertake the significant capital investments needed to enhance the reliability of the existing power systems. Thus, our national electric power system has become overstressed and is subject to potential failures. Technological advances need to be developed that utilize advances in communications and controls that enable automatic detection of power system problems and automatic reconfiguration of the electrical transmission and distribution grids to minimize potential impacts of any natural and human caused disasters.

The main goal of this project was focused on enhancing the reliability, security and economics of our national electric power system by pursuing two key objectives:

- Advance the technological awareness of integrating multi-agent controls and associated hardware (such as sensors and distributed resources) into an existing electric power system, and

- Assess the associated increase in effectively operating and controlling a next generation power system, such as the Circuit of the Future developed by Southern California Edison.

The Circuit of the Future Project by the SCE had a goal to “...design a futuristic distribution feeder from scratch, maximizing the use of cutting-edge and emerging technologies in a manner that will satisfy the long-term needs of customers in a manner better than existing designs.” See Reference [1.13]. This SCE CoF Project was designed around an existing $12 \mathrm{kV}$ distribution circuit located near San Bernardino, CA. The circuit will supply approximately 2,000 customers from a connection to the western US transmission grid at a $115 \mathrm{kV} / 12 \mathrm{kV}$ substation on the SCE system. See Figure 1.1.1 and Figure 1.1.2.

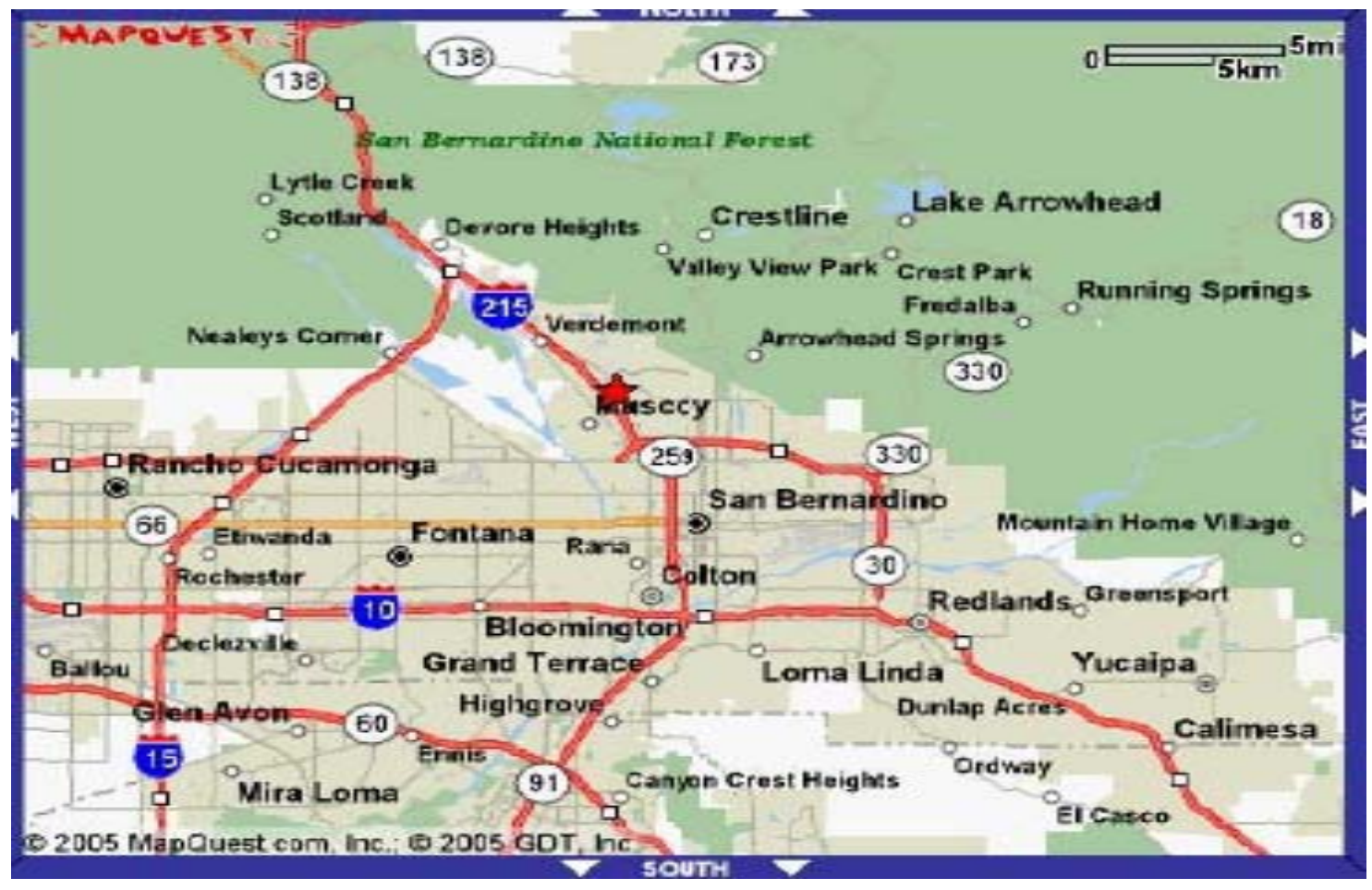


Figure 1.1.1. Geographical Location of SCE Circuit of the Future Project.

SCE (as part of a separately funded effort that coincided, in part, with this project) substantially renovated an existing electric power distribution circuit to test new technologies and to find ways to improve the quality of distribution system services. One reactive power device (initially a switched capacitor bank) was located at the far end of the circuit (relative to the transmission substation) to provide voltage support for loads on the circuit.

While the CoF Project does not currently include distributed generation, the circuit does include a point where DER/DG technologies may be connected for testing/evaluation. As such, the CoF could serve as a test bed for a variety of DER/DG technologies in the future.

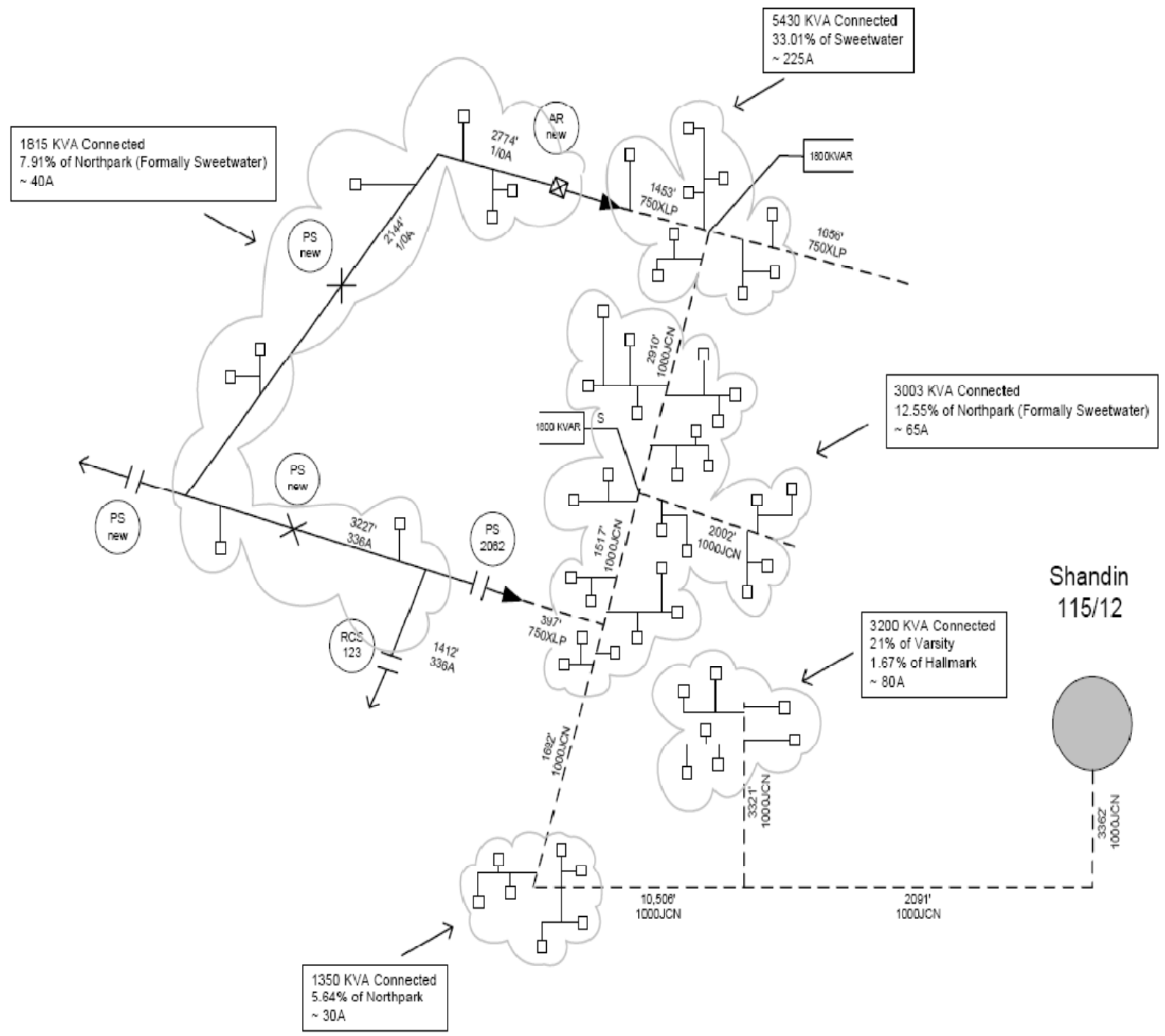

Figure 1.1.2. Schematic Diagram of SCE Circuit of the Future Project.

Leveraging the "Integrated Control of Next Generation Power Systems" Project's relationship with SCE and its CoF Project, control agents will be proposed and developed to form a coordinated multi-agent system. Through sensors and communications, a MAS (when integrated into an existing electrical power system) will monitor the circuit operating conditions 
and autonomously reconfigure the transmission and distribution grid to manage the flow of electricity and thus delivery of critical power. This integration of multi-agent controls and communication devices will be developed with a perspective on business benefits. See Figure 1.1.3. Both computer simulations and laboratory experiments will provide an evaluation of this proposed control method for next generation power systems. The main technical outcomes are expected to be improved protection, better reconfiguration and enhanced reliability of the electric power distribution system.

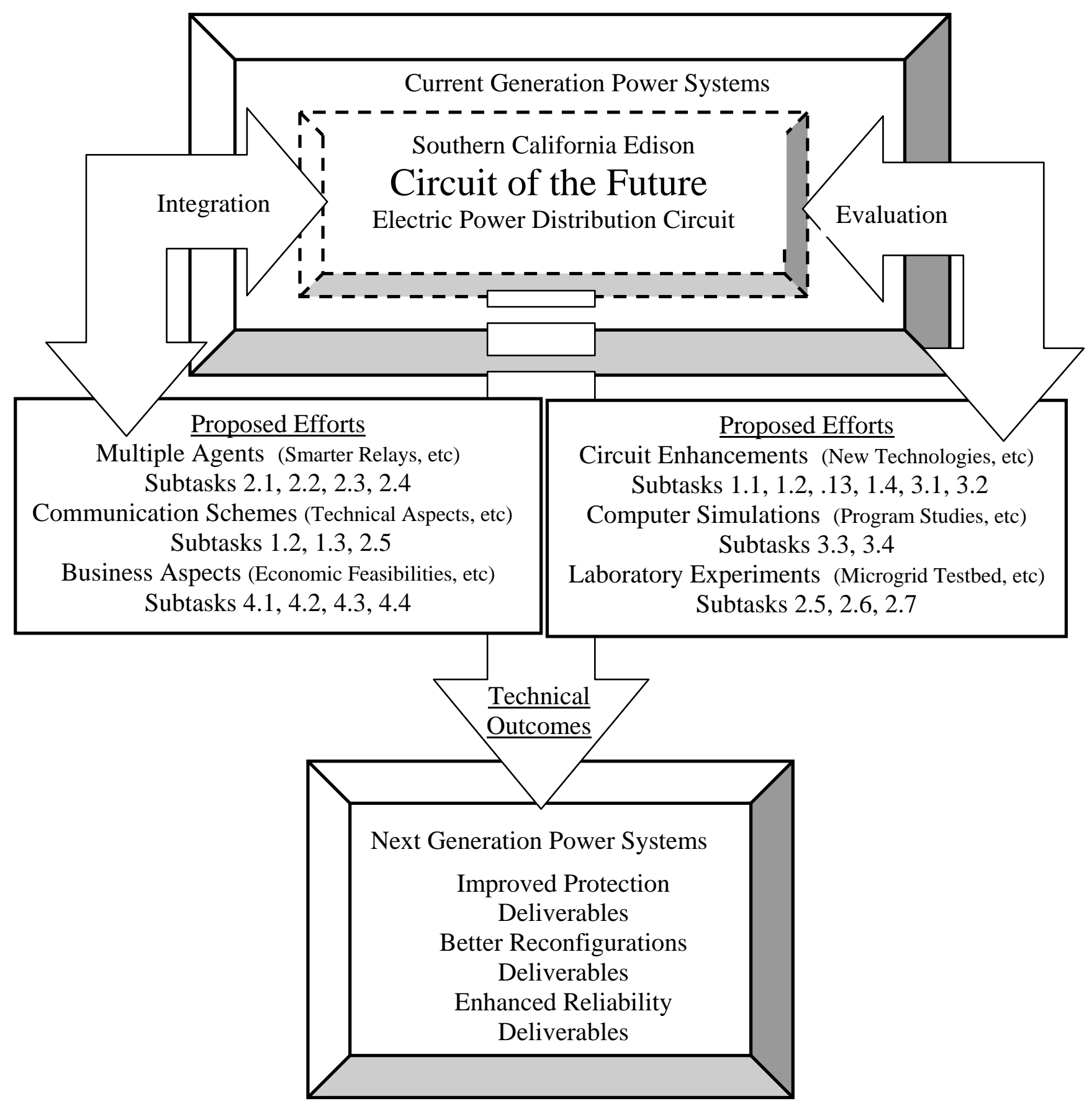

Figure 1.1.3. Block Diagram of Proposed Efforts and Technical Outcomes. 
Efforts to integrate a MAS and a communication scheme within the existing SCE CoF power distribution system will further enable our national electrical infrastructure to be more resilient to natural and other events that could otherwise result in blackouts.

\subsection{Compile List of Equipment Deployed and Planned on SCE CoF Grid}

This section first reviews some of the commercially available technologies that are currently deployed within SCE distribution circuits. These technologies include 1.2.1) Control and Protection Technologies and 1.2.2) Switching Technologies.

\subsubsection{Control and Protection Technologies.}

Control methodologies provide the necessary data acquisition, analysis, and corrective actions needed to maintain the electric system state within acceptable operating limits. They are primarily software-based algorithms that are nonfunctional unless properly integrated with system data and the appropriate control devices. Components of today's control methodologies include: protective relays, SCADA, distribution automation, feeder automation, software agents, sensors, control devices, and communications.

The technology of protective relays has significantly changed over the past years. The older electromechanical relays have been replaced by modern, solid-state, intelligent designs known as Intelligent Electronic Devices (IED).

SCADA systems are common at the transmission level and also exist at the distribution level in some areas. Distribution Automation (DA) and Feeder Automation technologies have also been integrated with SCADA to provide rapid reconfiguration of specific sections of the distribution system to minimize the impact of system faults and power quality disturbances on customers. DA provides the ability to monitor and operate devices that are installed beyond the substation, thereby optimizing station loading and reactive supply, monitoring equipment health, identifying outages, and enabling more rapid system restoration.

Today's control methodologies depend on data sensing and some form of data transmission. System sensors continue to depend on traditional large, high-cost instrument transformers that convert the high voltages and currents to a measurable level. The high cost of these instrument transformers currently limits the volume and granularity of data acquired and hence impacts the capabilities of the control methodologies that depend on the data. Low-cost replacements for instrument transformers, capable of readily interfacing with legacy and emerging communications systems, will be an important addition in support of advanced control methodologies.

The system sensors that measure watts and watt-hours, reactive volt-amperes (vars) and varhours, volts, amperes, power factor, etc. are evolving from the traditional electric/electromechanical design to a solid-state, electronic-based technology of higher accuracy and more intelligence. The widespread deployment of these intelligent electronic devices at the system, equipment, and consumer levels is needed to support the deployment of advanced control methodologies in the future. These IEDs are also needed to support other enterprise processes and technologies such as Asset Management, Work Management, and Outage Management.

Present control methodologies analyze the acquired data and determine corrective actions needed to keep the electric system within desired operating parameters. Necessary corrective actions are still accomplished using large electromechanical devices such as vacuum, oil and 
gas-insulated breakers, capacitor banks, regulators, transformer tap changers, reclosers, generators, and more recently FACTS devices.

\subsubsection{Switching Technologies.}

As with other electricity distribution systems around the country, three basic kinds of switches are used on the SCE grid. In terms of their functionality and operation in a circuit, these devices are commonly referred to as circuit breakers (also called fault interrupters), and loadbreak and non-loadbreak switches. A brief summary of each these types of switches follows. Efforts by this project to apply MAS technologies to enhance these devices will be discussed as part of Tasks 2, 3 and 4.

\section{- Circuit breakers.}

Circuit breakers or fault interrupters are devices that can act autonomously to interrupt (break) the flow of electric current in a circuit whenever a short circuit (fault) or overload occurs on the electric grid. Circuit breakers are also used for normal switching operations under load.

Because of the large amount of energy flowing in a circuit, an electric arc is generated when a circuit breaker opens. To prevent equipment damage, the arc must somehow be quickly extinguished or prevented (minimized). Older circuit breakers were equipped with an arc extinguishing mechanism (spring-loaded actuators to quickly separate the breaker contacts) to minimize arcing, or snuff out the arc with a blast of compressed air. In newer circuit breakers, the contacts are engulfed in an insulating fluid (oil), gas [such as Sulfur hexafluoride ( $\left.\mathrm{SF}_{6}\right)$ ], or a vacuum.

Automatic reclosers are basically fault interrupters (circuit breaker) with a reclosing relay. After this type of breaker trips (opens) due to a fault, the relay then 'recloses' the contacts to reenergize the circuit. If the fault is still there, the breaker trips again. This process can be repeated up to three more times until another downstream device (another recloser, sectionalizer, vacuum interrupter or fuse) operates to clear the fault and minimizing the number of customers that would otherwise be out of service. Also, since most faults on an overhead distribution system are temporary, reclosing technology significantly improves reliability by virtually eliminating long outages due to momentary faults.

Most existing fault interrupters and reclosers can be operated manually and remotely. Some types of non-automatic manually operated legacy units can be upgraded to facilitate remote control by retrofitting them with closing/opening coils.

When a short circuit occurs, reclosers can go through several open-close cycles before a fault is cleared. However, each reclose subjects grid components (such as transformers, conductors, etc.) to high inrush current, eventually shortening their life expectancy. To minimize this effect, S\&C Electric has developed a recloser, called an IntelliRupter, which can identify permanent faults without fully closing the breaker contacts thereby extending the useful life of grid components and improving power quality.

\section{- Loadbreak switches.}

While these switches cannot break short circuit (fault) current, they can interrupt typical line currents (up to the rating of the switch). In other words they can be operated under normal load. This provides an operational convenience (such as during an emergency or overload condition) to quickly de-energize a portion of the circuit or transfer load between adjoining circuits. Loadbreak switches are usually equipped with an arc extinguishing mechanism (some using a 
medium such as $\mathrm{SF}_{6}$ gas) to snuff out the electric arc produced during switch operation. Many of these switches can be operated manually or remotely.

\section{- Non-loadbreak switches.}

These types of switches (typically called air break or open air switches) do not have an arc extinguishing mechanism. Therefore, they cannot interrupt load or short circuit current, and can only be operated under no-load conditions. Non-loadbreak switches are less expensive than loadbreak or automatic types, are typically manually operated, and can be modified for remote operation with the addition of a motor unit.

This section now reviews commercially available technologies expected to be deployed on the SCE CoF distribution circuit. Devices planned for installation include 1.2.3) Energy and Power Conditioning Technologies, 1.2.4) Communication Technologies, and 1.2.5) Advanced Sensors and Metering. Efforts by this project to apply MAS technologies to enhance such equipment will be discussed as part of Tasks 2, 3 and 4 .

\subsubsection{Energy and Power Conditioning Technologies.}

During the course of this project, the SCE CoF (also referred to as the Avanti circuit) did not include distributed generation. However, the circuit will include a device (installed at the far end of the distribution circuit) that will supply reactive power for voltage support. The sections below describe some of the DER devices that could be included for the Avanti circuit, or similarly advanced distribution circuits.

- Reciprocating Diesel or Natural Gas Engines.

Reciprocating engines, developed more than 100 years ago, were the first among DG technologies. Both Otto (spark ignition) and Diesel cycle (compression ignition) engines have gained widespread acceptance in almost every sector of the economy. They are used on many scales, with applications ranging from fractional horsepower units that power small tools to enormous 60 MW baseload electric power plants.

Smaller engines are primarily designed for transportation applications, but can usually be converted to power generation with little modification. Larger engines are most frequently designed for power generation, mechanical drive, or marine propulsion. Reciprocating engines can be fueled by diesel or natural gas, with varying emission outputs. Almost all engines used for power generation are four-stroke and operate in four cycles (intake, compression, combustion, and exhaust). The process begins with fuel and air being mixed. In turbocharged applications, the air is compressed before mixing with fuel. The fuel/air mixture is introduced into the combustion cylinder and ignited with a spark. For diesel units, the air and fuel are introduced separately with fuel being injected after the air is compressed.

Reciprocating engines are currently available from many manufacturers in all size ranges. They are typically used for either continuous power or backup emergency power. Cogeneration configurations are available with heat recovery from the gaseous exhaust.

“The Installed Base of US Distributed Generation”, a report prepared by Resource Dynamics Corporation, reveals that many distributed generation units are already present in the electricity delivery system. According to the report, there are 12,300,000 DG units in the systems which are smaller than $5 \mathrm{MW}$ with a total installed capacity of $222 \mathrm{GW}$. Reciprocating engines compose $192 \mathrm{GW}$ of this total capacity. Most of these units are not connected to the grid and are 
being used as back-up generators. Considering that the total summer generation capacity in the US is $978 \mathrm{GW}$, the present distributed generation capacity is quite significant.

\section{- Microturbines.}

Microturbines are an emerging class of small-scale distributed power generation in the 30$400 \mathrm{~kW}$ size range. The basic technology used in microturbines is derived from aircraft auxiliary power systems, diesel engine turbochargers, and automotive designs. A number of companies are currently field-testing demonstration units, and several commercial units are available for purchase.

Microturbines consist of a compressor, combustor, turbine, and generator. The compressors and turbines are typically radial-flow designs, and resemble automotive engine turbochargers. Most designs are single-shaft and use a high speed permanent magnet generator producing variable voltage, variable frequency alternating current (AC) power. Most microturbine units are designed for continuous-duty operation and are recuperated to obtain higher electrical efficiencies.

- Combustion Gas Turbines.

Combustion turbines range in size from simple cycle units starting at about $1 \mathrm{MW}$ to several hundred MW when configured as a combined cycle power plant. Units from 1-15 MW are generally referred to as industrial turbines (or sometimes as miniturbines), which differentiates them both from larger utility grade turbines and smaller microturbines.

Although units smaller than $1 \mathrm{MW}$ exist, few have been installed in the U.S. Industrial turbines are currently available from numerous manufacturers. Historically, they were developed as aero derivatives, spawned from engines used for jet propulsion. Some, however, are designed specifically for stationary power generation or compression applications in the oil and gas industries. Multiple stages are typical and, along with axial blading, differentiate these turbines from the smaller microturbines described above.

Combustion turbines have relatively low installation costs, low emissions, and infrequent maintenance requirements. However, their low electric efficiency has limited turbines to be used primarily as peaking units and combined heat and power (CHP) applications. Cogeneration DG installations are particularly advantageous when a continuous supply of steam or hot water is desired.

\section{- Fuel Cells.}

There are many types of fuel cells currently under development in the $5-1000+\mathrm{kW}$ size range, including phosphoric acid, proton exchange membrane, molten carbonate, solid oxide, alkaline, and direct methanol. One company, International Fuel Cells/ONSI, currently manufacturers a $200 \mathrm{~kW}$ phosphoric acid fuel cell for use in commercial and industrial applications. A number of companies are close to commercializing proton exchange membrane fuel cells, with marketplace introductions expected soon.

Although the numerous types of fuel cells differ in their electrolytic material, they all use the same basic principle. A fuel cell consists of two electrodes separated by an electrolyte. Hydrogen fuel is fed into the anode of the fuel cell. Oxygen (or air) enters the fuel cell through the cathode. With the aid of a catalyst, the hydrogen atom splits into a proton $(\mathrm{H}+)$ and an electron. Direct current (DC) is produced as the proton passes through the electrolyte to the cathode and the electrons travel in an external circuit. At the cathode, protons combine with hydrogen and oxygen, producing water and heat. Fuel cells have very low levels of nitrogen oxides (NOx) and carbon monoxide (CO) emissions because the power conversion is an electrochemical process. The part of a fuel cell that contains the electrodes and electrolytic 
material is called the "stack", and is a major contributor to the total cost of a fuel cell system. Stack replacement is very costly but becomes necessary when efficiency degrades as stack operating hours accumulate.

Fuel cells require hydrogen for operation. However, it is generally impractical to use hydrogen directly as a fuel source; instead, it must be extracted from hydrogen-rich sources such as gasoline, propane, or natural gas. Cost effective, efficient fuel reformers that can convert various fuels to hydrogen are necessary to allow fuel cells increased flexibility and commercial feasibility.

- Photovoltaics (PV).

Photovoltaic systems are commonly known as solar panels. Photovoltaic (PV) solar panels are made up of discrete cells connected together that convert light radiation into electricity. The PV cells produce direct current (DC) electricity, which must then be inverted for use in an AC system. Current units have efficiencies of $24 \%$ in the lab and $10 \%$ in actual use, below the $30 \%$ maximum theoretical efficiency that can be attained by a PV cell.

Insolation is a term used to describe available solar energy that can be converted to electricity. The factors that affect insolation are the intensity of the light and the operating temperature of the PV cells. Light intensity is dependent on the local latitude and climate and generally increases as the site gets closer to the equator.

Photovoltaic systems produce no emissions, are reliable, and require minimal maintenance to operate. They are currently available from a number of manufacturers for both residential and commercial applications. Use of PV systems is slowly becoming more widespread as manufacturers continue to reduce installed costs and increase efficiency. Applications for remote power are quite common.

\section{- Wind Turbines.}

Windmills have been used for many years to harness wind energy for mechanical work like pumping water. Before the Rural Electrification Act in the 1920s provided funds to extend electric power to outlying areas, farms were using windmills to produce electricity with electric generators. In the U.S. alone, eight million mechanical windmills have been installed.

Wind energy became a significant topic in the 1970's during the energy crisis in the U.S. and the resulting search for potential renewable energy sources. Wind turbines, windmills dedicated to producing electricity, were considered the most economically viable choice within the renewable energy portfolio. Today, attention has remained focused on this technology as an environmentally sound and convenient alternative. Wind turbines produce electricity without requiring additional investments in infrastructure such as new transmission lines, and are thus commonly employed for remote power applications. They are currently available from many manufacturers and improvements in installed cost and efficiency continue.

Wind turbines are packaged systems that include the rotor, generator, turbine blades, and drive or coupling device. As wind blows through the blades, the air exerts aerodynamic forces that cause the blades to turn the rotor. As the rotor turns, its speed is altered to match the operating speed of the generator. Most systems have a gearbox and generator in a single unit behind the turbine blades. As with PV systems, the output of the generator is processed by an inverter that changes the electricity from DC to AC so that the electricity can be used.

\section{- $\quad$ NAS Battery and other Energy Storage Options.}

A sodium-sulfur (NaS) battery consists of liquid (molten) sulfur at the positive electrode and liquid (molten) sodium at the negative electrode as active materials separated by a solid beta 
alumina ceramic electrolyte. The electrolyte allows only the positive sodium ions to go through it and combine with the sulfur to form sodium polysulfides.

$$
2 \mathrm{Na}+4 \mathrm{~S}=\mathrm{Na}_{2} \mathrm{~S}_{4}
$$

During discharge, positive $\mathrm{Na}+$ ions flow through the electrolyte and electrons flow in the external circuit of the battery producing about 2 volts. This process is reversible as charging causes sodium polysulfides to release the positive sodium ions back through the electrolyte to recombine as elemental sodium. The battery is kept at about 300 degrees $C$ to allow this process.

NaS battery cells are efficient (about 89\%) and have a pulse power capability over six times their continuous rating (for 30 seconds). This attribute enables the $\mathrm{NaS}$ battery to be economically used in combined power quality and peak shaving applications.

$\mathrm{NaS}$ battery technology has been demonstrated at over 30 sites in Japan totaling more than 20 MW with stored energy suitable for 8 hours daily peak shaving. The largest NaS installation is a 6 MW, 8 hour unit for Tokyo Electric Power Company. Combined power quality and peak shaving applications in the U.S. market are under evaluation.

There are other kinds of batteries like, Vanadium Redox Battery (VRB), Zinc Bromide (ZnBr), Lithium Ion (Li-Ion), Lead-Acid batteries. A VRB is a flow battery with external tanks containing Vanadium aqueous solutions. Thus the number of hours of storage could be increased by simply increasing the size of the external tanks.

Ultracapacitors and Super Capacitors store electricity by physically separating positive and negative charges - unlike batteries which do so chemically. The charge they hold is like the static electricity that can build up on a balloon, but is much greater thanks to the extremely high surface area of their interior materials.

Ultracapacitors are very good at efficiently capturing electricity from regenerative braking, and can deliver power for acceleration just as quickly. With no moving parts, they also have a very long lifespan.

Ultracapacitor technology is promising. Considering the operational and economic advantages of ultracapacitors over batteries, they can replace the batteries at most of the applications in the near future.

\subsubsection{Communications Technologies.}

Various communication media are being used in today's electric grid, including copper, optical fiber, and power line carrier and wireless technologies. These communication technologies have enabled the deployment of substation automation (SA) at many facilities.

Power line carrier (PLC) technology has been in use for many years in the utility industry. Recently, broadband over power line (BPL) carrier technologies have been developed and successfully demonstrated on a pilot basis. Also, wireless technologies are currently being developed and demonstrated, but they have not yet penetrated the grid communications infrastructure on either the system or the user side. The current state of BPL and wireless technologies are discussed below.

\section{- BPL Technologies.}

Numerous vendors have developed and commercialized BPL technologies and are moving aggressively to market their products. The primary features of the various BPL technologies include:

- Utilizes and communicates with low- and medium-voltage distribution facilities

- Normally operates at $2-50 \mathrm{MHz}$ 
- Can interface the consumer at the $120 \mathrm{~V}$ outlet or via wireless Wi-Fi from the mediumvoltage line at the consumer's premise

- Can enable mobile data communications in wireless designs

- Achieves data rates of 20 Mbps with potential for up to $100 \mathrm{Mbps}$ or more

- Originally focused on Internet access and VOIP for consumers, is increasingly being deployed to meet utility needs for AMR, DER, DR, and consumer portal applications, as well as DA and video monitoring (primarily for security) applications and other high speed data needs on the system side.

Current limitations of BPL include:

- Transmission and substation communications do not utilize BPL technology because currently it is only deployable over low- and medium-voltage distribution facilities.

- It is claimed that some BPL technologies interfere with the HAM radio band. Techniques to coordinate with HAM radio have been developed and appear very effective in eliminating interference concerns.

- Technology has been developed, but deployment and integration with distribution facilities has been limited.

BPL has been demonstrated in over 30 pilot projects and trials, but it has not yet adequately penetrated the communications market as the lead candidate for supporting the integrated communications infrastructure for the modern grid. The next generation of BPL systems, now under development, promises lower cost, improved performance, and higher speed and are expected to support a wide range of utility applications.

\section{- Wireless Technologies.}

Various wireless technologies are emerging as possible candidates for the communication infrastructure of the modern grid. To date, few of them have made significant market penetration in either system or user side applications. Some of these technologies include:

- Radio (700MHz, $900 \mathrm{MHz}, 2.4 \mathrm{GHz}$ )

- Wi-Fi

- Wi-Max

- Next Generation Cellular

- Satellite (Ka-Band)

One of the most promising of these is Wi-Max, which is expected to range 25 miles. Demonstrations to compare the performance and economics of BPL and Wi-Max are planned in the future. Currently, however, wireless technologies do not play a major role in the electric grid communications infrastructure.

\subsubsection{Advanced Sensors and Metering.}

The requirements for advanced T\&D sensors placed on the line or in the substation that have been identified by EPRI and TVA are shown below:

- Low cost Sensor, installation, and System Cost

- Standoff inspection

- Low maintenance cost (without batteries)

- Inaccessible locations like energized conductor, Inside cabinets, on structures)

- Multiple applications like rounds inspection at high speed or online monitoring

- Feasible dimensions for application by being small in size compared to components assessed

- Should not create EMC concerns 
- Security

Examples of sensors that are being developed and tested by the EPRI and TVA can be found in a report titled "Development of Low Cost Battery-less Sensors for T\&D". One such sensor can measure the conductor temperature thereby allowing accurate dynamic rating of overhead lines, line sag, and thus line rating. In addition, it can measure the temperature difference between the conductor and the conductor splice or compression connecter, which normally is 20 degrees $\mathrm{F}$ cooler than the conductor itself. If there is no temperature difference, the compression connector is approaching conditions for failure. This sensor is critical as failures of splices (compression connectors) account for a large portion of sustained outages on the transmission system.

Another low cost sensor is being developed to detect insulator leakage current. This sensor is critical to determining when an insulator flashover is imminent due to contamination, including wind born salt mists in coastal areas of the country.

Today there are many deployments of automated meter reading (AMR) systems using slow speed communications and less than "state of the art" meters. These investments (unless they can be retrofitted to be compatible with the needs of the modern grid) may ultimately become "throw away" technologies". Without the appropriate standards, both companies and consumers will be hesitant to invest, fearing they will be left with stranded assets.

EPRI has done substantial conceptual work in the formulation of a Consumer Portal that embodies advanced metering, demand response and a number of nonutility services. One formal definition of a consumer portal is "a combination of hardware and software that enables two-way communication between energy service organizations and equipment within the consumers' premises."

The EPRI consumer portal project identified the functions that a portal needs to perform, but noted that those functions can be embodied physically in a number of places - the meter, a cable modem, a set-top box, a pole-top advanced metering infrastructure (AMI) data concentrator, a thermostat, etc.

\subsection{Study Deployed and Planned Equipment to be Integrated into SCE CoF Grid.}

This section provides a list of equipment that could be integrated into the SCE CoF grid. These technologies include 1.3.1) Emerging Distribution System Technologies, 1.3.2) Technologies Described in the Academic Literature related to Agent-Based Control of Distribution Systems, 1.3.3) The Multimodal Approach, 1.3.4) Communication Devices and Systems Currently Deployed, 1.3.5) IEC 61850 - Communication Networks and Systems in Substations and 1.3.6) Other Groups of Interest.

\subsubsection{Emerging Distribution System Technologies.}

The following technologies are either presently available or are being developed for possible deployment on various electricity delivery systems. Many of the devices are listed here as reference. A more detailed discussion is provided in Deliverable 3 which is included in the "Deliverables" section of this Final Report.

- Current Limiting Conductor (CLC).

- Distributed Series Impedance (DSI).

- HTS Synchronous Condenser--SuperVAR with 2G YBCO HTS wire. 
- HTS Fault Current Limiters (FCL) using 2G Wire.

- Composite Conductors.

- $1 \mathrm{G}$ and 2G HTS Cables.

- Advanced OLTC for Transformers.

- Advanced Substation and Distribution Automation.

- Fault Locator for Distribution Systems.

- Advanced Instrument Transformers.

- Advanced Signal Distribution.

\subsubsection{Technologies Described in Academic Literature Related to Agent-Based Control of Distribution Systems.}

Recent literature has provided documentation about research endeavors to apply agent-based control to distribution system. Some of these efforts (articles, papers, etc.) are mentioned in Deliverable 3 and referenced in Appendix 3.

Any effective multi-agent system (MAS) must include a robust high performance communication layer. In the course of evaluating technologies and specific hardware options for our MAS, it was determined that control and communication hardware should be integrated as much as possible to 1 ) reduce the required number of computing devices, i.e., reduce cost, 2) simplify development and maintenance requirements, and 3) increase the reliability of the system. Because of the highly complex nature of the power system communication network, it was also determined that an approach that seamlessly (plug-and-play) allows for and optimizes multiple modes of communication (wireless and wired) simultaneously would be best in terms of reliability, security, and cost. Off-the-shelf hardware was obtained and initial testing of basic multimodal communications was completed, as was the process of integrating this hardware with the power system simulator at WVU.

\subsubsection{The Multimodal Approach.}

Because power system communication and control networks are highly complex and heterogeneous, including myriad device types and communication modes and protocols, it was determined that no single mode of communication would meet our requirements. It was decided that a new paradigm was needed: multimodal communications. This approach to communication system design allows the use of multiple modes of communication simultaneously, even from a single device. Examples of modes include fiber optic cable, Ethernet, 2-wire twisted part (used in CANBUS), and wireless. Each of these modes offers some advantages and some disadvantages relative to other modes, as described in Table 13.1. Fiber optic communications, for example, provides the best bandwidth among all modes, supporting tens of gigabits per second of data. However, material and deployment costs are extremely high. Wireless communications, on the other hand, provides lower data rates, but is extremely inexpensive to deploy and maintain. Interestingly, power system communications are heterogeneous, composed of many different types of data with different constraints. Control data, for example, may require very low latency but also low data rates. Measurement data, on the other hand, may require much higher rates, but allow for higher latency, depending upon the specifics of the data. The result is that one can optimize communications on a per-device and/or per-data-type basis. Devices that require high rates, for example, can be fitted with fiber optic cable or Ethernet, perhaps with a wireless backup. Devices that do not require as much bandwidth can be fitted 
with inexpensive wireless devices. Importantly, our networking approach will be seamless with respect to modes. That is, the network will automatically use whatever communication modes are available in an optimal fashion, perhaps even simultaneously, to maximize reliability and security based on data constraints, e.g., delay and throughput.

Table 1.3.1 Advantages and Disadvantages of Communication Modes

\begin{tabular}{|c|c|l|l|l|c|}
\hline & Cost & Reliability & Bandwidth & Security & $\begin{array}{c}\text { Upgradability/ } \\
\text { Maintenance }\end{array}$ \\
\hline $\begin{array}{c}\text { Fiber Optic } \\
\text { Cable }\end{array}$ & $\begin{array}{c}\text { very } \\
\text { expensive }\end{array}$ & high & very high & excellent & very expensive \\
\hline Ethernet & $\begin{array}{c}\text { moderately } \\
\text { expensive }\end{array}$ & moderate & high & good & inexpensive \\
\hline Twisted Pair & inexpensive & moderate & low & good & inexpensive \\
\hline Wireless & inexpensive & high & moderate & moderate & very inexpensive \\
\hline
\end{tabular}

- General Hardware Considerations.

Smart devices, e.g., electronic relays, provide basic (serial) wired communication capabilities, while off-the-shelf radios provide basic wireless communication capabilities. However, microcontrollers, FPGA's and/or microprocessors are needed both for agent intelligence and for implementing the advanced communication protocols required for a complex multimodal $^{1}$ network. It was determined that the best practice for these networks would be to combine these intelligences into a single microcontroller. Specific hardware descriptions are available in Task 2. Because of the limited scope, the project was limited to the use of off-theshelf hardware. This places severe restrictions on the multimodal optimizations that can be performed $^{2}$, but one can still obtain benefits over purely wireless networks or separately optimized wired and wireless networks. For prototyping purposes, the wireless mode of our 802.11/15 radios will be combined with a twisted pair wired mode, e.g., Ethernet.

\subsubsection{Communication Devices and Systems Currently Deployed.}

Before describing the specific hardware obtained for MAS testing, a tabulation of some of the communication hardware used by various entities with an interest in agent networks for power systems is provided below.

Included below is a tabulation of the communication hardware and protocol for the power distribution control systems by S\&C, Intelligrid, Gridwise/Gridworks, ModernGrid Initiative

${ }^{1}$ A multimodal network is defined here as a network that automatically optimizes network traffic across whatever communication modes are available including, for example, fiber optic, wireless, Ethernet, twisted pair, USB. Some devices will have only one mode of communication but, in general, some devices will have multiple modes that can be optimized based on network conditions (disruptions), security requirements, and/or throughput/delay constraints.

${ }^{2}$ Software radio would provide the highest level of flexibility, but requires more initial expense and significantly more development and testing time. 
(MGI) and Infotiltiy. Also included is a description of the IEC 61850 protocol for Communications Networks and Systems in Substations as well as a tabulation of other groups with interest in communication hardware and protocol for the power distribution control systems.

- S\&C - IntelliTEAM IITM

\begin{tabular}{|c|c|}
\hline \multicolumn{2}{|c|}{$\begin{array}{l}\text { http://www.sandc.com/edocs_pdfs/edoc_024319.pdf } \\
\text { http://www.sandc.com/products/Energyline/IntelliTEAM/default.asp } \\
\text { http://www.sandc.com/edocs_pdfs/EDOC_025290.pdf } \\
\text { http://www.sandc.com/edocs_pdfs/EDOC_025077.pdf } \\
\text { http://www.sandc.com/edocs_pdfs/edoc_024450.pdf } \\
\text { IntelliTeam II Descriptive Bulletin 026576.pdf } \\
\text { Virtual Agent Based Distributed Intelligence.pdf } \\
\text { UtiliNetOverview_w.pdf }\end{array}$} \\
\hline \multicolumn{2}{|c|}{$\begin{array}{l}\text { S\&C has specified the control system hardware as EnergyLine } 5800 \text { Series with } \\
\text { IntelliTEAM II automated switch control. IntelliTEAM II has a few communication } \\
\text { options. Primary option is the UtilNET Series II WanGate wireless Radio. Also } \\
\text { supporting: RS-232, fiber, and direct modems. }\end{array}$} \\
\hline Communications Hardware: & $\begin{array}{l}\text { UtiliNet, UtiliNet Series II } \\
\text { UtiliNet Series } 3000 \\
\text { RS-232, RS-496 } \\
\text { Fiber } \\
\text { CDMA Modem } \\
\text { Ethernet } \\
900 \text { MHz Wireless Modem }\end{array}$ \\
\hline Communications Protocols: & $\begin{array}{l}\text { UtiliNet LAN Packet } \\
\text { DNP 3.0 } \\
\text { PG\&E SCADA (Cooper 2179) } \\
\text { Landis \& Gyr Telegyr 8979 } \\
\text { Motorola Intrac }\end{array}$ \\
\hline Most Recent Update Found: & $10-2006$ \\
\hline \multicolumn{2}{|c|}{$\begin{array}{l}\text { RadioShop Software Configuration } \\
\text { IntelliLINK Remote Setup Software }\end{array}$} \\
\hline
\end{tabular}

Agent Missions

o All Systems

- Maintain power to target line segments

- Avoid overloading during restoration

- Return the circuit to its normal configuration after restoration of faulted segments

o Systems with protective devices

- Ensure coordination during restoration

- Minimize momentary outages 


\section{- Intelligrid.}

\begin{tabular}{|c|c|}
\hline \multicolumn{2}{|c|}{$\begin{array}{l}\underline{\text { http://www.epri.com/IntelliGrid// }} \\
\text { http://publish.intelligrid.info/ } \\
\underline{\text { http://publish.intelligrid.info/HTML/IECSA_VolumeIV.htm }}\end{array}$} \\
\hline \multicolumn{2}{|c|}{$\begin{array}{l}\text { Multi-path Network } \\
\text { Primary Path and Secondary Path }\end{array}$} \\
\hline $\begin{array}{l}\text { Communications } \\
\text { Hardware: }\end{array}$ & $\begin{array}{l}\text { Multicast Routing } \\
\text { Ethernet } \\
\text { Bridges/Switches } \\
\text { Asynchronous Transfer Mode (ATM) } \\
\text { Global Positioning System (GPS) } \\
\text { Radio Frequency Identification (RFID) } \\
\text { Multiple Address (MAS) Radio } \\
\text { Spread Spectrum Radio System } \\
\text { Synchronous Optical Network (SONET) } \\
\text { Synchronous Digital Hierarchy (SDH) }\end{array}$ \\
\hline $\begin{array}{l}\text { Communications } \\
\text { Protocols: }\end{array}$ & $\begin{array}{l}\text { SNTP (Network Time Protocol) } \\
\text { User Datagram Protocol (UDP) } \\
\text { IEEE } 802 \text { MAC Addresses } \\
\text { Internet Protocol Version V4 (IPV4) } \\
\text { TCP/IP } \\
\text { IEC } 61850 \text { Substation Automation suite } \\
\text { IntelliGrid communications architecture } \\
\text { Distributed Network Protocol (DNP3) } \\
\text { IEC 60870-5 Telecontrol suite } \\
\text { Modbus } \\
\text { IEC 61968/61970 Common Information } \\
\quad \text { Model/GenericInterface Definitions } \\
\text { Universal Description, Discovery, and Integration (UDDI) } \\
\text { XML Protocol/Simple Object Access Protocol (SOAP) }\end{array}$ \\
\hline $\begin{array}{l}\text { Most Recent Update } \\
\text { Found: }\end{array}$ & $12-2005$ \\
\hline
\end{tabular}

- $\quad$ Gridwise/GridWorks.

http://www.gridwise.org/

http://www.gridwiseac.org/

session3_widergren.pdf

http://gwac.pnl.gov/cocoon/morf/gridwise/article//122

http://www.pnl.gov/news/2006/06-01.stm 


\begin{tabular}{|c|c|}
\hline \multicolumn{2}{|c|}{$\begin{array}{l}\text { See Article \#122 } \\
\text { Very general communication outline. Provide mostly guidelines and organization of } \\
\text { standards. }\end{array}$} \\
\hline $\begin{array}{l}\text { Communications } \\
\text { Hardware: }\end{array}$ & $\begin{array}{l}\text { Power Line Carrier Comm. System } \\
\text { IEEE } 802 \\
\text { IEEE } 1394\end{array}$ \\
\hline $\begin{array}{l}\text { Communications } \\
\text { Protocols: }\end{array}$ & $\begin{array}{l}\text { Internet(TCP/IP) } \\
\text { Web-services } \\
\text { VOIP } \\
\text { Integration } \\
\text { XML } \\
\text { BACnet }\end{array}$ \\
\hline $\begin{array}{l}\text { Most Recent Update } \\
\text { Found: }\end{array}$ & $09-2006$ \\
\hline Affiliate Companies: & $\begin{array}{l}\text { Areva - http://www.us.areva.com/ } \\
\text { Bonneville - htttp://www.bpa.gov/corporate/ } \\
\text { IBM - http://www.ibm.com/us/ } \\
\text { PJM - http://www.pjm.com/index.jsp } \\
\text { Schneider Electric - http://www.schneider-electric.com } \\
\text { Battelle - http://www.battelle.org/ } \\
\text { Electricity Innovation Institute (E2I) - http://www.e2i.org/ } \\
\text { NXEGEN - http://www.nxegen.com/ }\end{array}$ \\
\hline
\end{tabular}

GridWise ${ }^{\mathrm{TM}}$ is a vision for transforming the nation's electric power grid using advanced communications, automated controls and other forms of information technology. The effort includes assembly of a Board to articulate the architectural communication and control requirements and characteristics of a transformed energy system. The Board will be comprised of industry representation from electric power, economic markets, information technology, communications, end use, as well as policy and regulation. The architecture is to be used to encourage the creation of standards and policies consistent with the GridWise vision. The project will target and support such standards making bodies as the IEEE and the IEC for standards development. It will draw upon and engage related efforts such as being undertaken by various groups including OASIS and UN/CEFACT, W3C, IEC TC57, IEEE SCC21 1547.3, and E2I CEIDS.

\section{- $\quad$ Modern Grid Initiative (MGI).}

http://www.themoderngrid.org/resources.htm http://www.themoderngrid.org/docs/mg_systems_view_b1_v1.pdf http://www.themoderngrid.org/docs/Renz -Key Technology Areas 061206.pdf

MGI describes a flexible self-healing network, with high reliability and security. There doesn't seem to be a concrete decision on a communication standard. 


\begin{tabular}{|c|l|}
\hline Communications & BPL - PLC, Power Line Communications \\
Hardware: & Radio (700MHz, 900 MHz, 2.4 GHz) \\
& Wi-Fi \\
& Wi-Max \\
& Next Generation Cellular \\
& Satellite (Ka-Band) \\
& Fiber \\
\hline Communications & CIM \\
Protocols: & IEEE P1901 - PHY/MAC standards for BPL \\
& IEC 61850 \\
& IEC 60870-6-TASE.2 - Exchange of Data \\
& Between Control Centers \\
\hline Most Recent Update & 10 - 2006 \\
Found: & Utility Communications Architecture (UCA) International \\
\hline & Users Group \\
\hline
\end{tabular}

BPL: Numerous vendors have developed and commercialized BPL technologies and are moving aggressively to market their products. The primary features of the various BPL technologies include:

- Utilizes and communicates with low- and medium-voltage distribution facilities

- Normally operates at $2-50 \mathrm{MHz}$

- Can interface the consumer at the $120 \mathrm{~V}$ outlet or via wireless Wi-Fi from the mediumvoltage line at the consumer's premise

- Can enable mobile data communications in wireless designs

- Achieves data rates of $20 \mathrm{Mbps}$ with potential for up to $100 \mathrm{Mbps}$ or more

- Originally focused on Internet access and VOIP for consumers, is increasingly being deployed to meet utility needs for AMR, DER, DR, and consumer portal applications, as well as DA and video monitoring (primarily for security) applications and other high speed data needs on the system side

Presently, limitations of BPL include:

- Transmission and substation communications do not utilize BPL technology because currently it is only deployable over low- and medium-voltage distribution facilities.

- It is claimed that some BPL technologies interfere with the HAM radio band. Techniques to coordinate with HAM radio have been developed and appear very effective in eliminating interference concerns.

- Technology has been developed, but deployment and integration with distribution facilities has been limited.

BPL has been demonstrated in over 30 pilot projects and trials, but it has not yet adequately penetrated the communications market as the lead candidate for supporting the integrated communications infrastructure for the modern grid. The next generation of BPL systems, now under development, promise lower cost, improved performance, and higher speed and are expected to support a wide range of utility applications. 
- Infotility.

\begin{tabular}{|l|l|}
\hline $\begin{array}{l}\text { http://www.electricdistribution.ctc.com/pdfs/distributed\%20intelligent cohen non- } \\
\text { proprietary.pdf } \\
\text { http://www.matteroffact.com/portfolio/mof_CaseStudy3.pdf }\end{array}$ \\
\hline $\begin{array}{c}\text { Builds on Gridwise Architecture and ConEdison's 3G: Distribution System } \\
\text { of the Future. }\end{array}$ \\
\hline $\begin{array}{c}\text { Communications } \\
\text { Hardware: }\end{array}$ & Gridwise \\
\hline $\begin{array}{c}\text { Communications } \\
\text { Protocols: }\end{array}$ & IEC 68150 \\
\hline $\begin{array}{c}\text { Most Recent } \\
\text { Update Found: }\end{array}$ & $04-2005$ \\
\hline
\end{tabular}

\subsubsection{IEC 61850 - Communication Networks and Systems in Substations.}

\begin{tabular}{|c|c|}
\hline \multicolumn{2}{|c|}{ http://www.61850.com/ http://nettedautomation.com/books/sa-book.html\#order } \\
\hline $\begin{array}{c}\text { IEC61850 has defined a prioritized communication protocol using a generic } \\
\text { communication interface. Currently a combination of TCP/IP and Ethernet are the core } \\
\text { communication hardware. }\end{array}$ \\
\hline $\begin{array}{c}\text { Communications } \\
\text { Hardware: }\end{array}$ & Ethernet \\
\hline $\begin{array}{c}\text { Communications } \\
\text { Protocols: }\end{array}$ & TCP/IP \\
& MMS - Manufacturing Message Specification \\
& IEC 61970 Common Data Model (CIM) \\
& Exchange of Data Between Control Centers \\
\hline Most Recent Update & 06 - 2005 \\
Found: & Utility Communications Architecture (UCA) \\
& International Users Group \\
\hline
\end{tabular}

IEC 61850 is the international standard for substation automation systems. It defines the communication between devices in the substation and the related system requirements, and supports all substation automation functions and their engineering. Different from that of earlier standards, the technical approach makes IEC 61850 flexible and future-proof. The ideas behind IEC 61850 are also applicable in areas of automation such as control and monitoring of distributed generation. 
Service Model: In a substation automation system, some data are time-critical but some are not. Time-critical data include, for example, trip signals for circuit breakers and start signals for disturbance recorders. An example of non-time-critical data is the changes of device settings. Time-critical data need to be sent quickly while non-time-critical data have lower transmission priority. IEC 61850 defines a set of generic services which can meet the requirements of all kinds of anticipated data in a substation with regard to speed of transfer, accuracy and security.

Generic Object Oriented Substation Event (GOOSE) is a fast service. The high speed is achieved by means of connection-less communication and the security of data transfer is assured by repeating the message many times. File Transfer is a service for moving large data blocks such as programs and fault records.

The data models and services are mapped onto a real communication stack. The stack comprises the Manufacturing Message Specification (MMS), Transmission Control Protocol/Internet Protocol (TCP/IP) and Ethernet. Data models and services models do not change much with time, but communication technology may. When communication technology does change, the data models and service models will be mapped onto a new real communication stack. This minimizes changes not only in the standard but also in the substation automation system.

\subsubsection{Other Groups of Interest.}

The Advanced Grid Application Consortium (GridApp) http://gridapp.org/

\section{Concurrent Technologies Corporation (CTC)}

Funded by DOE to help "transition utility best technologies and best practices into broader use by Consortium member utilities to support grid modernization"

\section{California Energy Commission Public Interest Energy Research (CEC-PIER)} http://www.energy.ca.gov/pier/

The PIER program of the California Energy Commission has the mission to conduct public interest energy research that seeks to improve the quality of life for California citizens by developing environmentally sound, safe, reliable, and affordable electricity services and products.

National Energy Technology Laboratory http://www.netl.doe.gov/

In 2005 the National Energy Technology Laboratory launched an effort called "Creating or Developing the Modern Grid" for describing the characteristics and technologies of an intelligent grid.

Energy Future Coalition http://www.energyfuturecoalition.org/

Smart Power Grid. U.S. Department of Energy http://www.oe.energy.gov/

U.S. Department of Energy, Office of Electricity Delivery and Energy Reliability 
Power System Engineering Research Center (PSERC) http://www.pserc.wisc.edu/

PSERC is an association of universities working with industry to find innovative solutions to the challenges facing the electric power industry and to educate the next generation of power industry engineers.

Galvin Electricity Initiative http://www.galvinelectricity.org/

The Galvin Electricity Initiative is an organization, founded by Bob Galvin, to create an actionable blueprint for transforming the U.S. electricity supply and service infrastructure into a resilient and adaptable system that can perfectly meet the needs of the rapidly evolving digital economy and society.

Consortium for Electric Reliability Technology Solutions (CERTS) http://certs.lbl.gov/

CERTS is an organization funded by the Department of Energy and the California Energy Commission to research, develop, and disseminate new methods, tools, and technologies to protect and enhance the reliability of the U.S. electric power system and the functioning of a competitive electricity market.

New York State Energy Research and Development Authority (NYSERDA) http://www.nyserda.org/

The NYSERDA is a public benefit corporation that intends to assist in the modernization of New York's aging transmission and distribution system to meet the needs of the 21st century.

European Union Framework Program on Sustainable Energy Systems. http://fp6.cordis.lu/

The European Union has organized its research portfolio in an "Electricity Networks of the Future" platform that seek to increase the efficiency, safety and reliability of the European electricity transmission and distribution system by transforming the current electricity grids into an interactive (customers/operators) service network and to remove obstacles to the large-scale deployment and effective integration of distributed and renewable energy sources.

\subsection{Establish Requirements for Equipment to be Implemented in SCE CoF Grid.}

The requirements for the equipment to be implemented in the SCE CoF Grid will need to comply with both SCE Practices and Industry Practices. A tabulation of these requirements, as provided by Charlie Vartanian at SCE, follows below. Included are the Utility Grid Interconnection Specifications in Table 1.4.1, the Utility Grid Conditions That May Influence Inverter Design in Table 1.4.2, the Inverter Performance Requirements for Grid Support Protocol in Table 1.4.3, and the Communication and Control Interface Protocol in Table 1.4.4. 
Table 1.4.1. Utility Grid Interconnection Specifications.

\begin{tabular}{|c|c|c|}
\hline $\begin{array}{l}\text { Grid Interconnection } \\
\text { Specifications }\end{array}$ & SCE Criteria & Industry Criteria \\
\hline Voltage Rating & $\begin{array}{l}\text { SCE's common secondary } \\
\text { distribution voltages: } 120 / 240 \mathrm{~V} \\
\text { tapped single phase. 120/208, } \\
\text { 240/480 V, 277/480 V 3-phase. } \\
\text { SCE's Common Primary } \\
\text { Distribution Voltages, } 12 \mathrm{KV} \text { and } \\
\text { 4,160 V } \\
\text { Allowable normal, or steady } \\
\text { state, voltage variation range is +/- } \\
\text { 5\% from nominal. }\end{array}$ & $\begin{array}{l}\text { Not reported. } \\
\text { ANSI C.84.1 provides national } \\
\text { standards }\end{array}$ \\
\hline Current Rating & $\begin{array}{l}\text { No ampere based ratings. } \\
\text { Practical limit based on typical } \\
\text { circuit protective overload targets: } \\
720 \text { A Phase, } 80 \text { A Neutral }\end{array}$ & Not reported \\
\hline Power Rating & $\begin{array}{l}\quad \text { Limits per interconnection } \\
\text { related tariffs: Rule } 21 \text { (retail } \\
\text { tariff), } 15 \% \text { of circuit peak load. } \\
\text { Peak circuit loads no more than } \\
600 \mathrm{~A} \text {, or } 12 \mathrm{MVA} \text { at } 12 \mathrm{KV} \text {. }\end{array}$ & $\begin{array}{l}\text { Federal Energy Regulatory } \\
\text { Commission (FERC) } \\
\text { Small Generator } \\
\text { Interconnection Procedure ( SGIP) } \\
\text { small gen. max. capacity is 20MW }\end{array}$ \\
\hline Harmonic Content & $\begin{array}{c}\text { Based on IEEE- } 519 \\
\text { Limits for Odd Harmonic } \\
\text { Multiples } \mathrm{h}<11,4 \%, 11<\mathrm{h}<17,2 \% \\
17<\mathrm{h}<23,1.5 \%, 23<\mathrm{h}<35,0.6 \% \\
35<\mathrm{h}, 0.3 \%, \mathrm{THD} \leq 5 \%\end{array}$ & IEEE-519 \\
\hline $\begin{array}{c}\text { Regulatory } \\
\text { Requirements }\end{array}$ & $\begin{array}{l}\text { Specified in terms of power } \\
\text { factor: } 0.90 / 0.90 \text { lead/lag }\end{array}$ & Not reported \\
\hline $\begin{array}{l}\text { Regulatory } \\
\text { Requirements }\end{array}$ & $\begin{array}{l}\quad \text { CPUC Rule } 21 \text { provides } \\
\text { regulatory technical requirements } \\
\text { for customer generation. Rule } 2 \\
\text { provides technical requirements for } \\
\text { load. }\end{array}$ & Not reported \\
\hline $\begin{array}{c}\text { Voltage } \\
\text { Spikes(Sags) }\end{array}$ & $\begin{array}{cc}\text { Maximum } & -25 \% \text { Instantaneous } \\
& -20 \% \text { for } 20 \text { cycles } \\
& -10 \% 10 \text { sec- } 3 \text { min } \\
\end{array}$ & $\begin{array}{l}\text { NERC Criteria same as SCE's } \\
\text { for system fault/outage related } \\
\text { spikes. }\end{array}$ \\
\hline $\begin{array}{l}\text { Power Surges, } \\
\text { Withstand }\end{array}$ & $\begin{array}{c}\text { Duty withstand location } \\
\text { specific. No general utility criteria. }\end{array}$ & Not reported. \\
\hline $\begin{array}{l}\text { RF Conduction } \\
\text { Limit }\end{array}$ & $\begin{array}{c}\text { No specific SCE Criteria for } \\
\text { customer load or generation. May }\end{array}$ & Not reported. \\
\hline
\end{tabular}


Table 1.4.2. Utility Grid Conditions That May Influence Inverter Design.

\begin{tabular}{|c|c|c|}
\hline Grid Conditions & SCE Conditions & Industry Conditions \\
\hline Power Surges & $\begin{array}{l}\text { Power surges are limited by } \\
\text { available Fault Duty. SCE system } \\
\text { can typically deliver } 10-30 \mathrm{kA} \text { at } \\
\text { distribution substations. Duty is } \\
\text { typically lower by factor of } 10 \text { or } \\
\text { more out on the circuit. }\end{array}$ & Not reported. \\
\hline Voltage Spikes & $\begin{array}{l}\text { Voltage Sags much more } \\
\text { common than spikes. Most } \\
\text { recorded sags are no lower than - } \\
20 \% \text { from nominal, for less than } 30 \\
\text { seconds. Majority no lower than - } \\
10 \% \text { of nominal. Recorded average } \\
\text { frequency of all sags }<20 \text { per year. }\end{array}$ & $\begin{array}{l}\text { Similar to SCE data for } \\
\text { national EPRI DPQ project stats } \\
\\
\text { RELEVANT TO SPIKES: } \\
\text { Per UL 1741, Dielectric } \\
\text { Voltage Withstand Test : a unit } \\
\text { shall withstand for } 1 \text { minute } \\
\text { w/out breakdown AC rms test } \\
\text { potential } \\
\text { (44.1.1. b) “1000 volts plus } \\
\text { twice the maximum secondary } \\
\text { circuit voltage between a } \\
\text { secondary circuit operating at } \\
\text { more than } 50 \text { volts and dead } \\
\text { metal parts." }\end{array}$ \\
\hline Electrical Noise & $\begin{array}{l}\text { "Noise" of concern is waveform } \\
\text { harmonic distortion (vs. transients } \\
\text { imposed on waveform). } \\
\text { For all monitored SCE } \\
\text { locations: } \\
\text { (CKV - Insert stats from EPRI } \\
\text { DPQ) }\end{array}$ & $\begin{array}{l}\text { Refer to EPRI DPQ Report, } \\
\text { Project's recorded harmonics } \\
\text { highly attenuated over } 17^{\text {th }} \\
\text { multiple. }\end{array}$ \\
\hline Other Factors & ported. & No \\
\hline
\end{tabular}


Table 1.4.3. Inverter Performance Requirements for Grid Support.

\begin{tabular}{|c|c|c|}
\hline $\begin{array}{c}\text { Inverter } \\
\text { Performance }\end{array}$ & SCE Conditions & Industry Conditions \\
\hline $\begin{array}{c}\text { Real and Reactive } \\
\text { Power } \\
\text { Compensation }\end{array}$ & $\begin{array}{l}\text { Reactive compensation is the product of } \\
\text { interest. } \\
\text { Up to } 4 \text { MVAR in } 500 \text { KVAR increments } \\
\text { would be a useful amount of reactive } \\
\text { compensation to be used for distribution } \\
\text { circuit support on SCE's system. }\end{array}$ & Not reported. \\
\hline $\begin{array}{l}\text { Power Factor } \\
\text { Correction }\end{array}$ & $\begin{array}{l}\text { The preferred target for power factor } \\
\text { correction at primary distribution would be } \\
\text { the maximum Rule } 2 \text { specified power factor } \\
\text { limit that customers are allowed to deviate; } \\
0.90 \text {. This is consistent with the power factor } \\
\text { capability requirements for generators per } \\
\text { Rule } 21 \text {. }\end{array}$ & Not reported \\
\hline $\begin{array}{l}\text { Harmonic } \\
\text { Correction }\end{array}$ & $\begin{array}{l}\text { Quantity of injected current needed to } \\
\text { clean up utility voltage distortion is very } \\
\text { location (SCD) dependent. } \\
\text { But in general, design target should } \\
\text { support the utility requirement of no more } \\
\text { than } 3 \% \text { voltage distortion. } \\
\text { Based on EPRI DPQ recorded data, } \\
\text { canceling-harmonic currents may not be } \\
\text { needed above several odd multiples to keep } \\
\text { utility within the utility's 3\% THDv } \\
\text { requirement }\end{array}$ & Not reported \\
\hline Voltage Regulation & $\begin{array}{l}\text { PF regulation } 0.9 \text { lead/lag, or } \\
\text { Voltage regulation }+/-5 \%\end{array}$ & Not reported. \\
\hline Line Balance & $\begin{array}{l}\text { Up to the allowable customer deviation of } \\
3 \% \text {. }\end{array}$ & Not reported. \\
\hline Scalability & $\begin{array}{l}\text { DER Load Flow analysis indicates useful } \\
\text { VAR increments of } 500 \mathrm{kVAR} \text { (typically } 1 / 2- \\
1 \% \text { v-delta impact), with total capacity of } \\
1000 \text { - } 4000 \text { KVAR, for primary distribution } \\
\text { voltage support. }\end{array}$ & Not reported. \\
\hline
\end{tabular}


Table 1.4.4 Communication and Control Interface Protocol.

\begin{tabular}{|c|c|c|}
\hline Comm. and Control & SCE Practices & Industry Practices \\
\hline Communication & $\begin{array}{l}\text { Most of the communications used by } \\
\text { SCE on the distribution system is done with } \\
\text { the CellNet UtiliNet, radio system. } \\
\text { It can handle a number of protocols, but } \\
\text { mostly is using DNP through an RS-232 } \\
\text { interface at this time. In the future, another } \\
\text { communications channel may be put in place } \\
\text { through the AMI project, but it is too soon in } \\
\text { the process to know what it will be. }\end{array}$ & $\begin{array}{l}\text { Some vendors and } \\
\text { industry consortia } \\
\text { (such as EPRI) } \\
\text { advocating IEC } 61850 \\
\text { Standard for Substation } \\
\text { Communication }\end{array}$ \\
\hline Control & $\begin{array}{l}\text { For circuit connected devices, primary } \\
\text { control protocol is manual or remote } \\
\text { switching in/out of service based on central } \\
\text { operator control. } \\
\text { Less common local/device 'automatic' } \\
\text { control is almost exclusively based on } \\
\text { feedback schemes that use sensing of } \\
\text { connected-bus voltage, and less frequently, } \\
\text { bus frequency. } \\
\text { Inverter should plan on local/autonomous } \\
\text { control scheme, with potential for future } \\
\text { integration with centralized computer- } \\
\text { processor based reactive device management. }\end{array}$ & Not Reported \\
\hline Future Changes & $\begin{array}{l}\text { The FERC/Energy Policy Act driven } \\
\text { Advanced Metering Initiative will impact } \\
\text { both communications and control. }\end{array}$ & Not reported \\
\hline
\end{tabular}




\section{Task 2.}

\section{Multi-Agent Systems.}

\subsection{Introduction.}

Efforts under this task will build upon the control agent methodology (CAM) developed by APERC for the U.S. Navy to enable the electrical systems of future all-electric ships to survive various military combat fault and damage scenarios. Since ship electrical systems typically operate on direct current, these previously developed methodologies only addressed unidirectional power flow.

In order to provide benefit to a modern electric grid, work under this task shall adapt the unidirectional DC smart agent technology to control alternating current and multi-directional power flow.

\subsubsection{Subtasks for Task 2 .}

This task has seven subtasks as follows:

2.1. Adaptation of the previously developed unidirectional DC control agents to multidirectional AC power systems, including development of supporting mathematical theory to overcome the unidirectional limitations of graph theory employed by these agents in their reconfiguration schemes,

2.2. Evaluation of a control agent's technical performance for properly managing power flow for 'real world' grid operating scenarios, including worst case events,

2.3. Assessment of the ability of the control agents to interface/interact with existing T\&D protective, control and communications devices,

2.4. Development of a design to readily integrate the control agents into existing $T \& D$ protective, control and communications apparatus and operating strategies,

2.5. Integration of a communications software component and the development of a list of offthe-shelf communications equipment to be used to build a prototype of protocol testing,

2.6. Demonstration of multi-agent concepts in a laboratory environment using electric circuits, and

As part of their collaborative effort during this project, SCE will provide support on subtasks 2.2, 2.3 and 2.4 as follows: i) model 'real world' scenarios that compare system performance with and without CAM/MAS, ii) perform additional analysis to determine the impacts of MAS across system network levels, and iii) develop the necessary circuit model and additional upstream system models, and execute the analysis.

\subsubsection{Deliverables for Task 2 .}

This task has two deliverables. The first deliverable, Deliverable 5, is a white paper report on the General Concepts for a Multi-Agent System. The second deliverable, Deliverable 7, about MAS Demonstrations has two parts. Deliverable 7.1 is a Report on Simulation Studies of a MAS for electric power systems. Deliverable 7.2 is a Report on Laboratory Tests of a MAS for electric power systems. 


\subsubsection{Multi-Agent System for Power Distribution System.}

In this section, a brief definition of Multi-Agent System (MAS) is given as well as a reason for applying it to power systems. An agent has been defined as "an autonomous computational entity such as software program that can be viewed as perceiving its environment through sensors and acting upon this environment through its effectors”.

So, a multi-agent system is composed of several agents interacting with each other to achieve a global goal. Hence, this type of technology can allow us to distribute and localize the control of power systems. By incorporating intelligence at the device level the reliability of the system should improve dramatically since there is no single point of failure as compared to the centralized control.

For example, by incorporating an agent into a simple relay, it turns the relay into an intelligent entity that can decide for itself, and talk to other components rather than acting as a simple switching device.

Especially in this era, where a power system is so complex and so large, sometimes the solution for a relay is not just cutting the power, for instance in case of a high current caused by a DG penetration, but to reroute the power.

Communication is the most important characteristic of a multi-agent system. It has to be simple and universal.

In this project, the agent communication language ACL by FIPA (Foundation for intelligent agents) is adopted.

\subsection{Adapt Unidirectional Control Agents to Multidirectional AC Power Systems.}

The application of control agents to next generation power systems will involve particular challenges pertinent to the needs and characteristics of AC electrical systems.

The multi-agent design for power distribution system reconfiguration that is based on the artificial immune system algorithm is implemented on a model of the SCE distribution feeder known as the CoF. The project is designed to test new hardware and automation technologies for circuit construction.

The system consists of 66 buses, one substation, two distributed generators and 14 residential loads with some capacitor banks scattered throughout the grid as shown in Figure 2.1.1 and Figure 2.1.3. The system is divided into three zones connected via controllable switches. In case of faults, these switches are operated to change or reconfigure the system in order to feed the loads and restore the power in the isolated areas.

In the following example, a line fault is simulated between node number 1205 and 1006 as shown in Figure 2.1.1. As soon as the fault occurs, AgentNodes at nodes 1205 and 1006 start communicating the current measured and decide to isolate the line. One can see from Figure 2.1.1 that the region which has been affected by the fault and the load at node 1013 has lost power. These agents then report the location of the fault to the Section Agent localized in that area of the circuit and a reconfiguration strategy is applied by collaboration with SwitchAgent\#4 to restore power to the load at node number 1013. Figure 2.1.2 shows the agents communication in the JADE (Java Agent Development) platform, and Figure 2.1.3 depicts the final solution and the new target reconfiguration. 


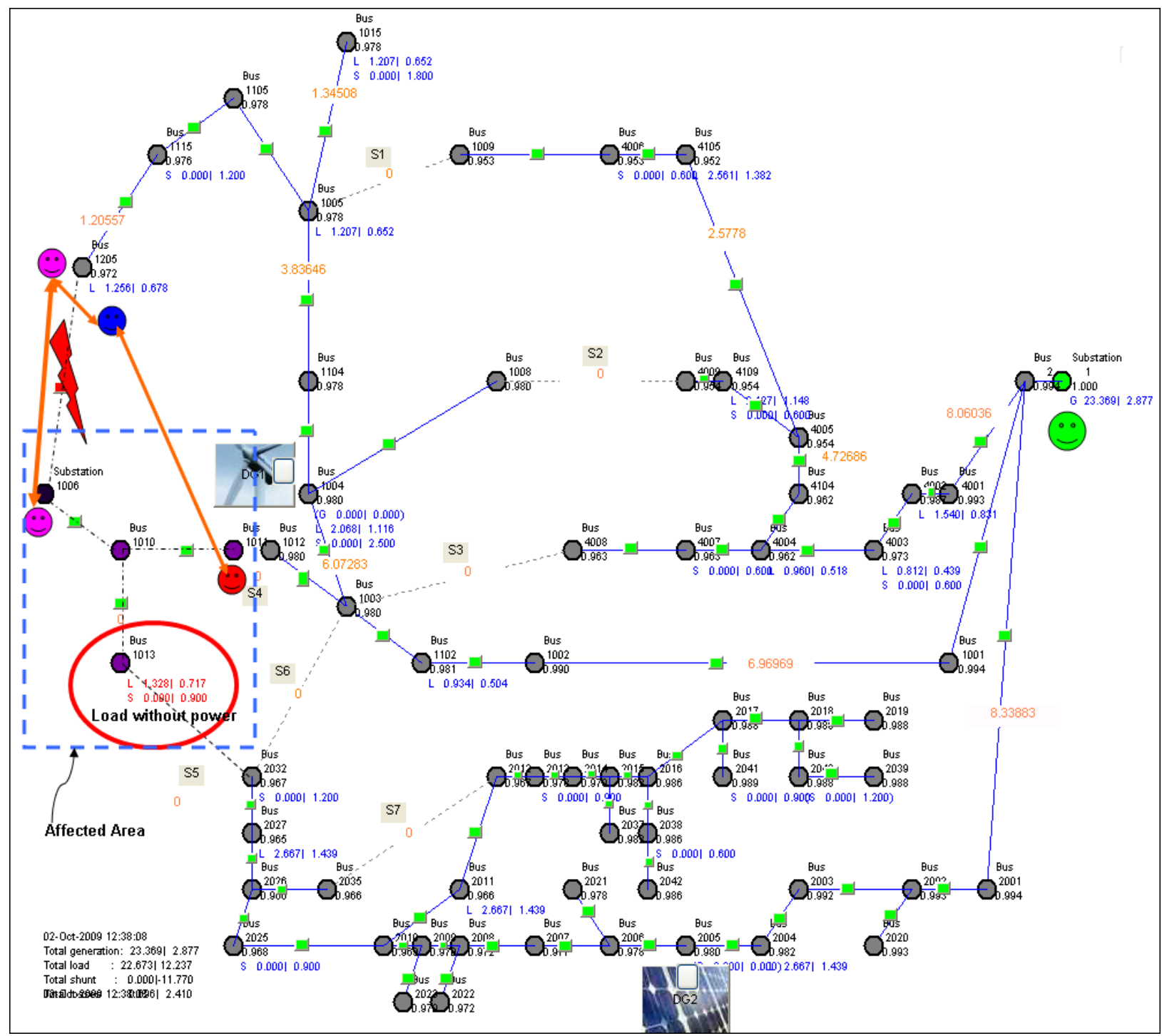

Figure 2.1.1. Model of SCE CoF Distribution System.

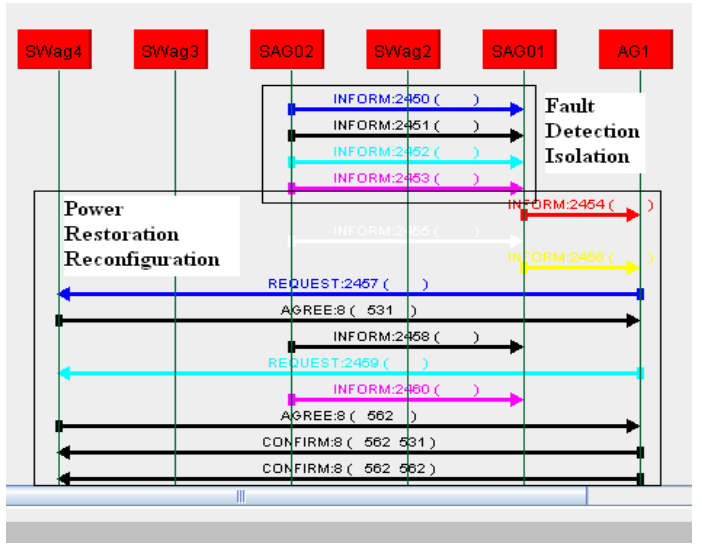

Figure 2.1.2. Agent Communication Following a Fault in the SCE CoF System. 


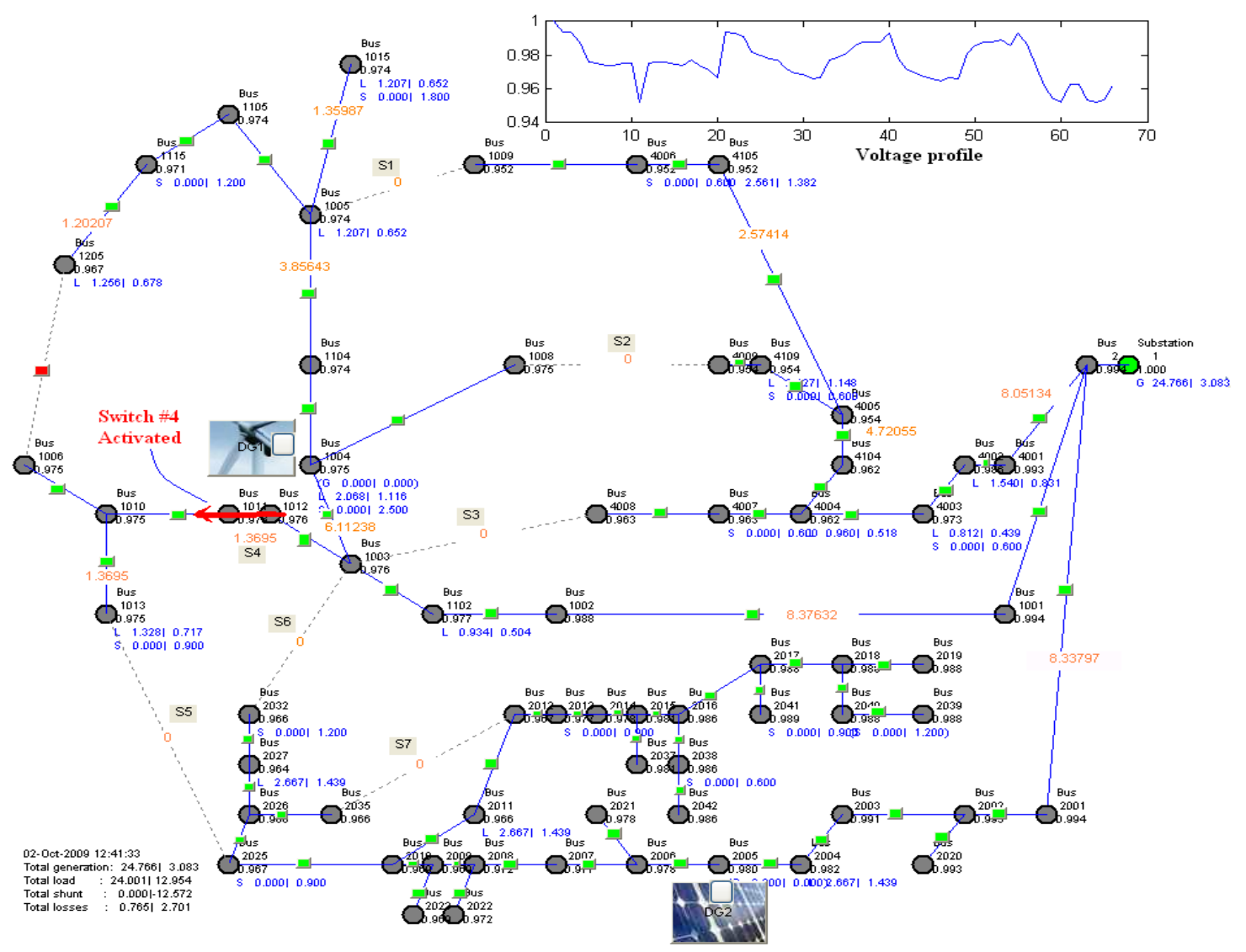

Figure 2.1.3. Target Reconfiguration of SCE CoF Distribution System.

The phase A current at the fault location is shown in Figure 2.1.4, clearly indicating the sudden increase in the current due to the fault.

Figure 2.1.5 shows the phase current seen at node \#1006. It is noticed that the fault is not seen at this location since the power is flowing from node 1205 to node 1006 . The phase current of the load at node \#1013 is shown in Figure 2.1.6. It was noticed that this load/region lost power when the agents isolated the line. Power was restored to the load after the agents performed the circuit reconfiguration.

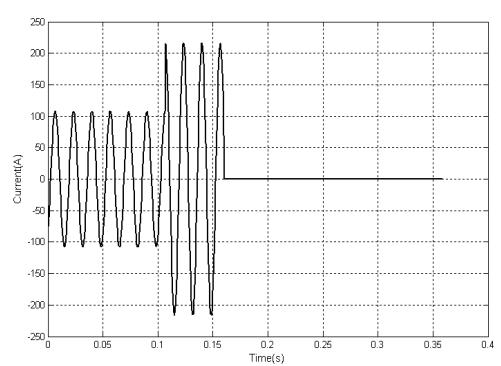

Figure 2.1.4. Phase A current at node \#1205.

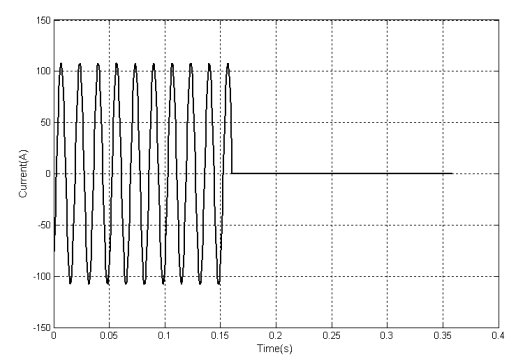

Figure 2.1.5. Phase A current at node \#1006. 


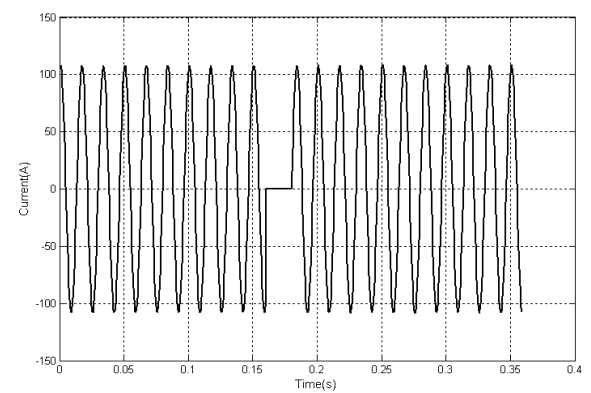

Figure 2.1.6. Current at Load \#1013.

\subsection{Evaluate Agent Technologies for Managing Real-World Grid Scenarios.}

In this subtask, an attempt was made to detect and prevent the voltage violations that typically occur in a power system due to variations in system load. The detection and subsequent improvement of the voltage is based on agent technology. The agent communication has been developed in JADE, which is a FIPA compliant platform. Bus Agents (BA) and Static VAR Compensator (SVC) Agents (SA) are developed, which detect the violations in voltage excursion, and stabilize the voltage by coordinated action. The SCE CoF was implemented in InterPSS, an open source Java based power system simulator. The results of the tests (conducted using a five bus system) show that, using coordinated agent communication, voltage violations are effectively detected and subsequently corrected.

\subsubsection{A Multi-Agent Coordinated Strategy.}

This research presents using multi-agent technology for the detection and elimination of voltage violations that typically occur in a power system due to load variations. Multi-agent systems, with their proactive behavior and communication capabilities, could play a vital role in helping to maintain power system voltages within specified limits. The objective of this research was to develop a multi-agent coordinated strategy wherein, given a specified voltage threshold for each bus (or node), the Bus Agents and the SVC Agents would detect the impending violation and react to change the SVC settings to maintain appropriate voltage levels (on the various buses) by injecting more reactive power into the system.

Today, FACTS devices are considered to be very useful in controlling the voltage and power flow in a power system. Typically, each FACTS device is assigned a primary zone of control and a key objective, which may be to control some disturbance, or to improve the overall stability of the power system. However, it should be noted that the influences of FACTS devices are not restricted to one bus or line. Since changing the voltage at a certain bus also changes the voltage at the other buses in the grid, the coordination of FACTS devices is very necessary. FACTS devices could also act in supporting adjacent devices in the network, to the extent permitted by the specifications of the device. To gain the maximum advantage of FACTS devices in a power system, effective coordination with other devices, loads, and generation sources is crucial. Failure to do so may affect the dynamic stability of the power system. So, for both steady state and dynamic operation of power systems, controlling and coordinating FACTS devices is essential. 


\subsubsection{Software.}

A. InterPSS - InterPSS is an open source project, with a simple yet powerful, Internet technology based Power System simulation framework. The open and loosely coupled system architecture provides a great advantage as the components and functionalities developed in InterPSS can be easily plugged into other integrated test systems. InterPSS uses the modern object oriented and network friendly language of Java for its platform. The scalability and portability of Java could be of vital use in system simulation on a distributed basis. InterPSS runs on multiple platforms like the Windows, Linux and UNIX systems, the only requirement being the availability of the Java Runtime Environment.

The InterPSS architecture is illustrated in Figure 3.2.10 on page 53. . At the center of the architecture is the Power System simulation model, which is based on an Object oriented model. Unified Modeling Language is used for the model, and the ECLIPSE framework is used to generate the source code. Since it is an open source framework, it can be easily maintained and modified by a team of volunteers, thus establishing the design goal. The InterPSS simulation framework uses a set of simulation objects (such as Network Topology, Network Classes, Bus Classes and Branch Classes) to create the various components of a power system. In addition, DC load flow, Short Circuit and Transient Stability analysis can be run. InterPSS also provides a graphical design tool with options to drag and drop buses and circuit branches, and create a network.

B. Java Agent Development Framework (JADE) - JADE is an open source Java based middleware for the development of distributed multi agent systems. JADE, which was developed by TILAB (Telecom Italia Laboratories), consists of three agents at startup, namely the AMS (Agent Management System), the DF (Directory Facilitator) and the ACC (Agent Communication Channel). The AMS provides white-page and life-cycle service, maintaining the directory of agent identifiers (AID) and agent state. Each agent must register with an AMS in order to get a valid AID. The Directory Facilitator (DF) is the agent that provides the default yellow page service in the platform. The Transport System, also called Agent Communication Channel (ACC), is the software component controlling all the exchange of messages within the platform, including messages to/from remote platforms. JADE's architecture is based on a modular structure, in which a platform is split into a number of containers, which may run on different machines.

A single special (Main) container must always be active in a platform, and all other containers register with the Main as soon as they start. By using the backup features of JADE, services can be duplicated on every computer. In JADE, the agent functions are implemented in behaviors that run recursively in the agent to implement the function of the agent. Each agent can have many behavior pools.

\subsubsection{Voltage Profile Improvement using MAS.}

Two Agents (namely the Bus Agent and the SVC Agent) are introduced into the system and the individual BA's monitor the voltage of their respective buses, namely the load or "PQ" buses. If voltages fall below the threshold of 0.95 per unit (p.u.), the $B A$ communicates with $S A$, to increase the SVC setting. Subsequently, the load flow is run using the modified SVC value, thus improving the voltages of the PQ buses. For this project, simulations were performed on the sample 5 bus system shown in Figure 2.2.1. 


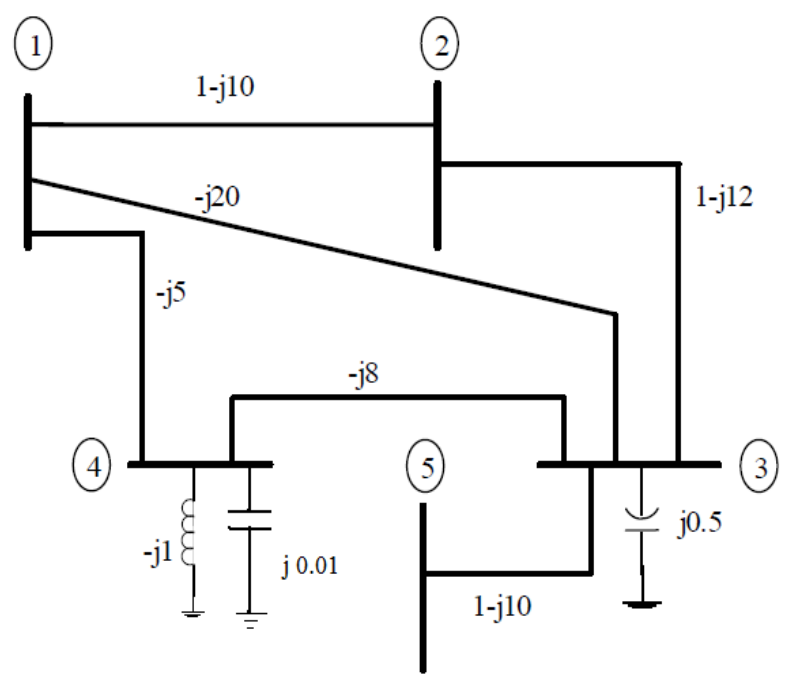

Figure 2.2.1. Sample Test System

\subsubsection{Status of System Buses.}

The test system consists of five buses. Bus 1 is a slack bus, Buses 2 and 5 are generator or "PV" buses and Buses 3 and 4 are PQ buses. The load profile of Bus 3 is shown in Figure 2.2.2.

LOAD PROFILE

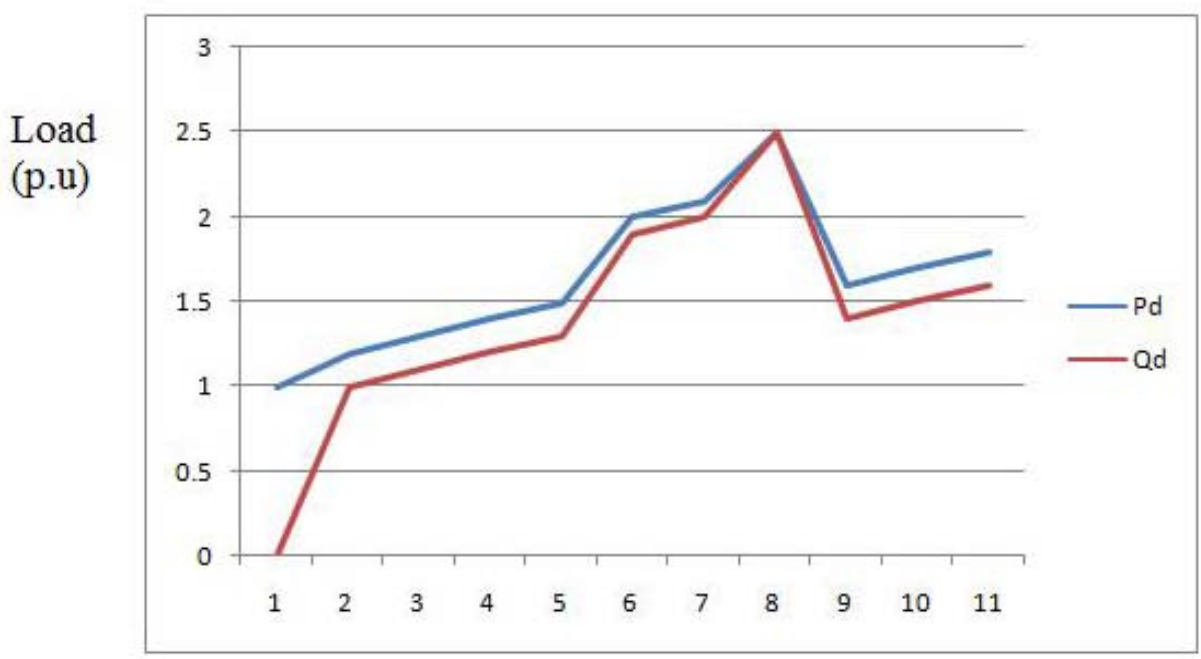

Figure 2.2.2. Load Profiles of Load at Bus 3

The load profile for 24 hours on Bus 3 is interpolated to obtain loading every 5 minutes. The varying nature of the load profile indicates that the buses may be subjected to sudden increases or decreases in load, as in the case of a real system. The power flow module of InterPSS runs every five minutes. Without agent action the voltage violations occurred on buses 3 and 4 as shown in Figure 2.2.3. With MAS the violations are detected and removed by coordination between the $B A$ and $S A$. The improved voltages of buses 3 and 4 are also shown in Figure 2.2.3. 


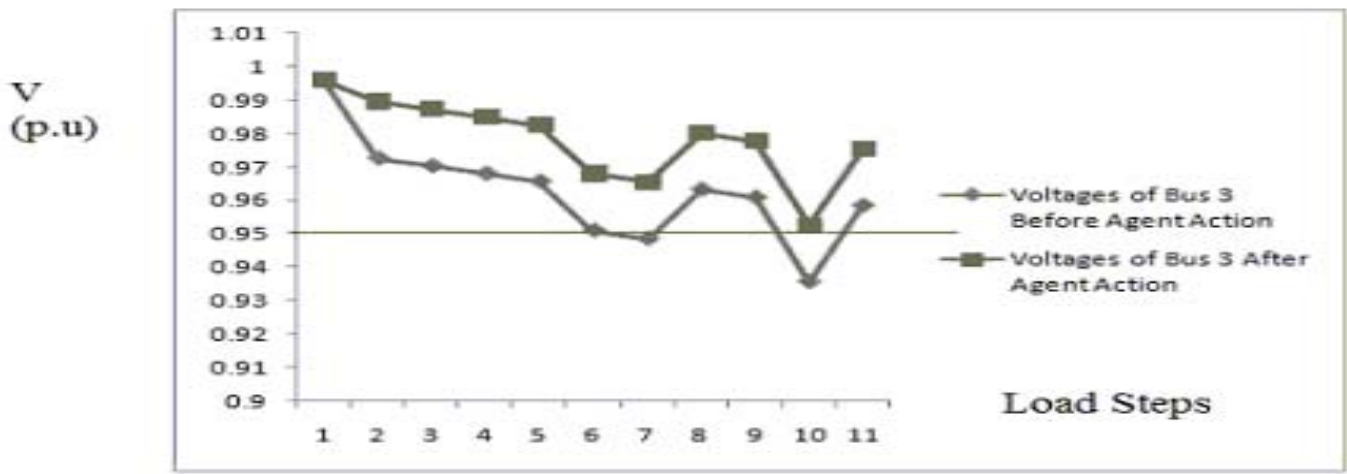

Figure 2.2.3 a) Bus 3 Voltages before and after Multi-Agent System Intervention

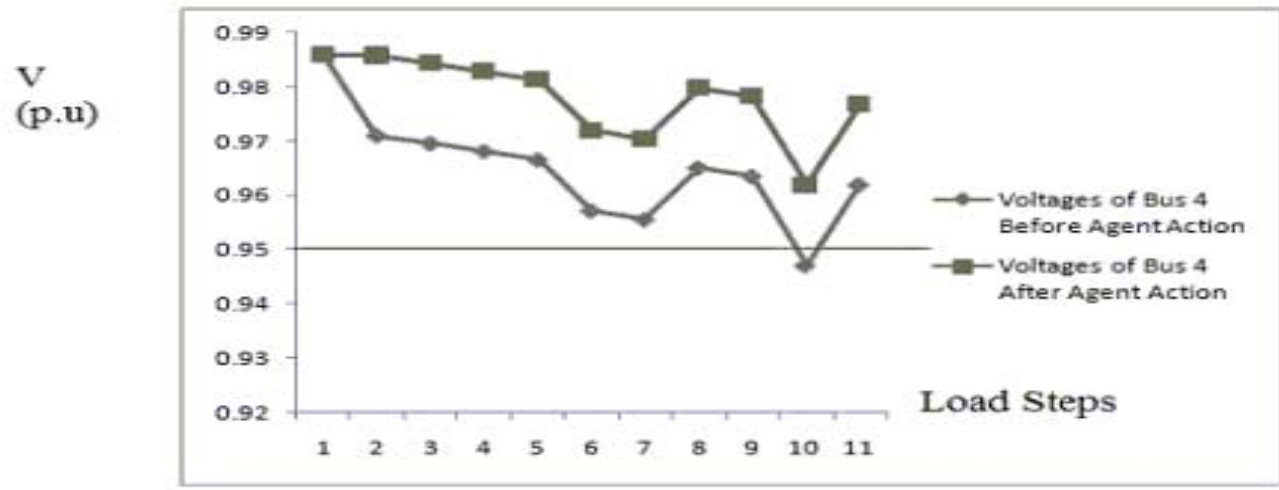

Figure 2.2.3 b) Bus 4 Voltages before and after Multi-Agent System Intervention

\subsubsection{Results of Simulation Study.}

From the simulation results, it can be seen that the voltage violation is effectively detected by the Bus Agent, and subsequent improvement is carried out by the SVC Agent. By using JADE for agent communication and InterPSS for the power system simulation, the performance of the agents was verified in the simulation. The scope for future work in this regard could be to introduce multiple parallel conversations among the agents, and to implement the strategy on a bigger scale and use real bus data, such as the Circuit of the Future.

\subsection{Assess Ability of Control Agents to Interface/Interact with Grid Devices.}

Agent technology has been readily used for distributed intelligent automated control. Intrinsically distributed, highly reactive, and multipartite systems are the most suitable candidates for the application of this technology. Owing to their capabilities, several agent based solutions have been proposed in the power systems area. Multi-agent systems have been applied for solving problems such as reconfiguration, restoration, fault identification, diagnosis, and power system protection. Agents have not only been used to interact with power system devices but also to interface and integrate standalone systems.

Agents are the main functional entities in these solutions for distributed intelligent control. By bringing intelligent solutions closer to the problem, agent systems provide increased reliability and quick problem solving. The agents interact with their environment through 
sensors and actuators. Specifically, in terms of power systems, environment means the power grid and associated devices. When deployed in this environment, the agents interact with all kinds of grid devices for the purpose of monitoring, reconfiguration, restoration, fault identification, and fault isolation.

Agent technology has been used for distributed power restoration using peer-to-peer communication. Such restoration systems perform isolation of faulty line sections and restoration of power to the un-faulted segments. During restoration the agents can also perform several important tasks like preventing line and source overloads, managing information sharing among devices, controlling the return to normal configuration, etc. Such tasks can only be accomplished through interactions between the agent system and the grid devices. For true automation agents have to interact with and monitor loads, power sources, switches, circuit breakers, and other devices as required. Effective and efficient interaction among different intelligent devices is necessary to achieve consistent results.

Power systems typically have sensors distributed at appropriate locations on the grid. These sensors provide raw data from various subsystems for evaluation and control of various dynamic events happening on the system. An agent based solution can have several agents interacting with these sensors to retrieve and process data. In addition to interaction with sensors, agents can also hold (store) the operation states for different devices/equipment. This helps in monitoring equipment condition (health) and alerting when maintenance is required.

In a power system, faults are detected by protective relays which then help isolate the faulty equipment and/or circuit segments so as to prevent the fault from affecting the entire system. Detecting faults requires information from the different devices and sensors dispersed on the system. This information can be obtained through the communication between agents which are used for collecting data from local and remote devices. For collecting remote data, an agent can either communicate with other agents in the system or can move itself closer to the data and function required. Each step of the protection process will involve agents interacting with each other and with several grid devices. This interaction will generate all the information necessary to perform the task at hand. In this manner, agent based control can be used to intelligently automate power system restoration, protection, maintenance, reconfiguration, fault isolation, etc. Whatever the functionality, agents will need to interface with different grid devices to collect real time data, such as voltage and current values, to work out an effective strategy for appropriately accomplishing the task at hand.

Much research effort has been (and continues to be) put into the automation of power systems. Several feeder automation tools have been introduced over the years. For example, medium voltage automatic source-transfer switchgear capable of restoring power without human intervention was recently introduced by S\&C Electric Company. Automated fault isolation is being provided by reclosers and breakers. Fuses can also be considered intelligent automated devices as they intrinsically react to the flow of excessive amounts of current. All these devices, in one way or another, manifest a combination of intelligent control and interaction with different grid devices thus minimizing human intervention.

To further enable automation and interaction of agent based control with grid devices, special modules or controls will be needed to interface with devices such as electronic relays, breakers and reclosers. The agents can then coordinate the operation of automated switches, breakers, and reclosers to achieve the desired level of automation. A very important step towards the automation of power systems is the use of intelligent electronic devices (IED). An IED is a microprocessor based controller of circuit breakers, transformers, capacitor banks, and other 
power system equipment. Common types of IEDs include protective relays, load tap changer controllers, circuit breaker controllers, capacitor bank switches, recloser controllers, voltage regulators, etc. IEDs can be deployed with an agent to perform different functions/operations. Such agents can then be used to sense currents and voltages to detect and isolate faults, restoring as much load as possible.

Interaction among agents, among different devices, and between agents and devices will also require a reliable communications network. Different kinds of communication infrastructures have been used with different agent based systems. Examples include, Ethernet LAN, SONET (Synchronous optical networking), Controller-area network (CAN), dedicated communication links between remote terminals and control centers, dedicated utility intranet, peer to peer communication networks, fiber optic networks, etc.

\subsection{CoF Distribution System Modeled using Graph Theory.}

To help restore power to customers following a fault, some feeders in the system have several interconnecting tie switches to neighboring feeders. Configuration alteration may be performed by changing the status of network switches (open/close) in such a way that normality is always re-established after the operations are completed. The reconfiguration of distribution feeders consists of changing the system topology by opening and closing switches with the objective of delivering power to each load as much as possible.

The distributed agent concept needs to be complemented by an appropriate framework for implementing automatic reconfiguration. Graph theory provides a formal basis to represent the distributed control system and to develop discrete algorithms for an energy management solution. Graph models are often used to represent networks. In the study of this project, a power system network is modeled as a graph $G$ with a single source node. Each node of graph $G$ (other than the source) represents either a load or a bus, or a distributed board; and each edge of $G$ represents a physical connection by power lines. One of the distribution circuits studied during this project is SCE distribution feeder called the Circuit of the Future, which is represented in the schematic shown in Figure 2.4.1.

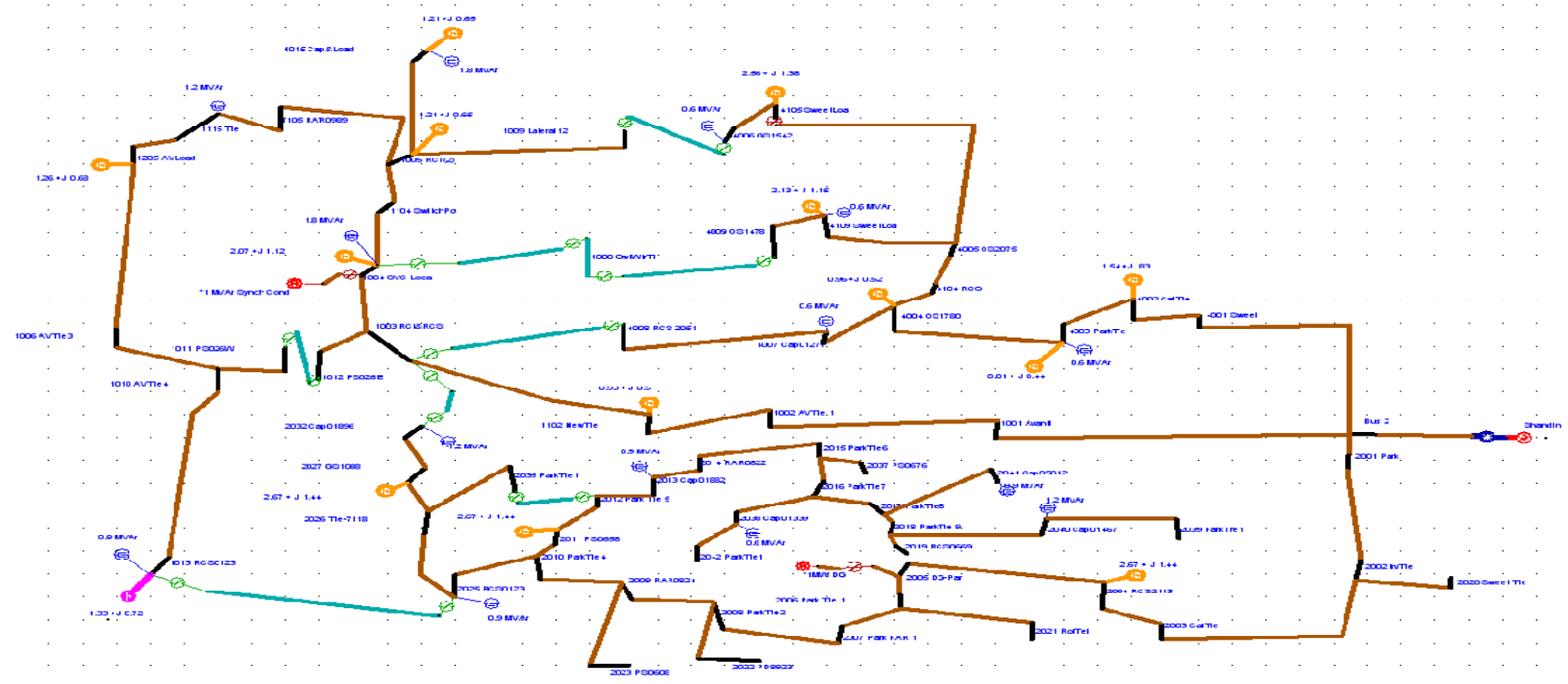

Figure 2.4.1. SCE Circuit of the Future. 
The depicted network can be simplified to the graph model shown by Figure 2.4.2. The solid lines in the diagram represent active (in use) physical power line connections, with the dotted lines representing inactive (power line connections that are not in use), but can be activated by the opening or closing of appropriate switches.

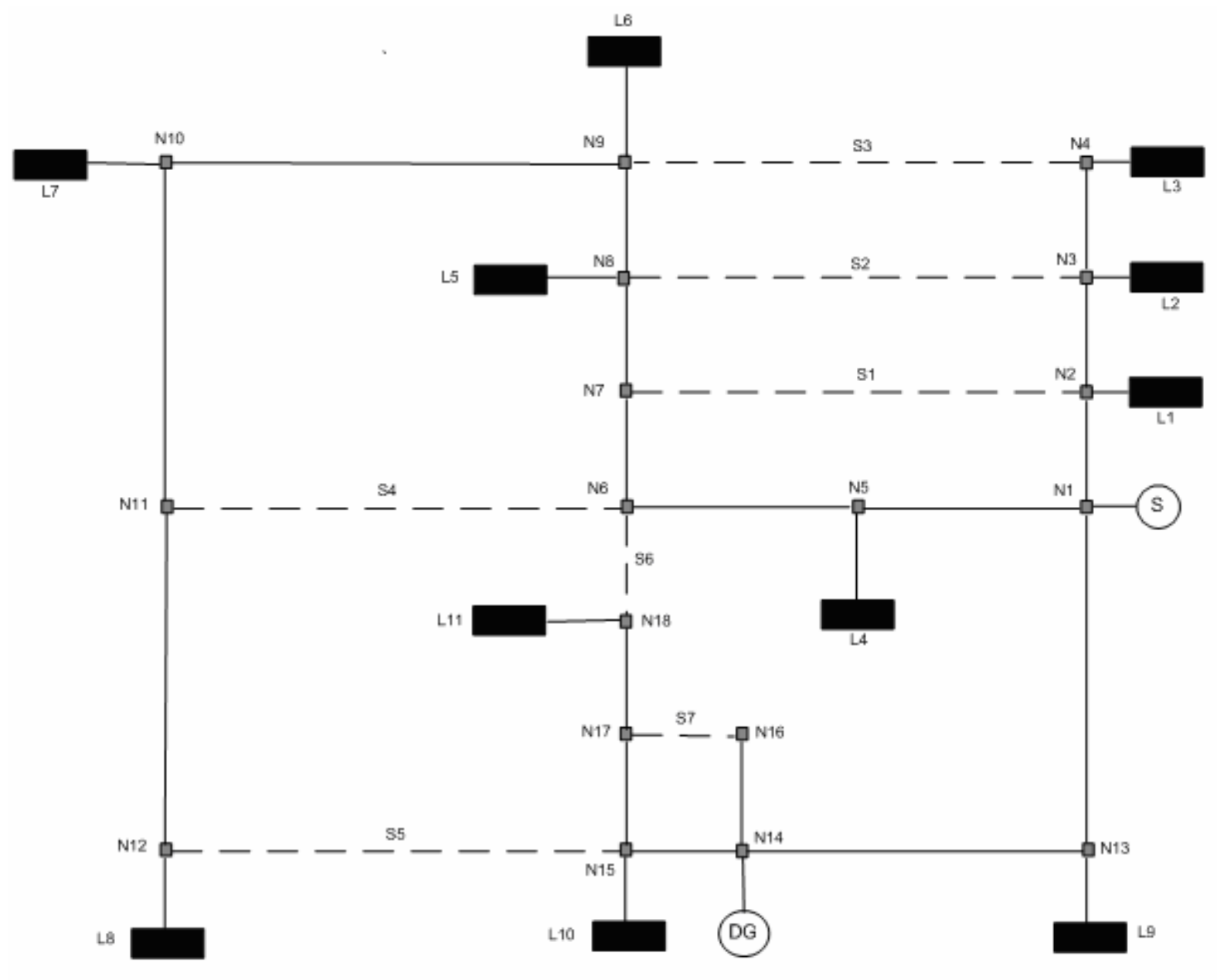

Figure 2.4.2. System Graph.

Such a depiction of the power system network also induces a graph model to represent the inter-relationship among the agents deployed in the power system. Agents deployed to monitor the power source and the performances of the loads will also be represented by corresponding nodes. The edges then indicate that each agent can negotiate and get immediate information from its neighboring agents. Depending on the nature of the problem, the relationship among agents deployed in the system can also be represented by an independent graph, where agents that can directly negotiate a decision for the corresponding devices are linked by edges in this graph.

Once the graph models are set up, graph theory and graph algorithms can then be applied to study the feasible ways that can be used for the desired power system reconfiguration. Such approaches have been successfully applied in several other projects, such as in References [2.9], [2.10], [2.11] and [2.12]. 
In Reference [2.13], graph models and graph algorithms were successfully deployed in the power network reconfiguration in conjunction with algorithms for detecting the power system reconfiguration termination. Simulations indicated that such approaches are very promising and effective.

During this project, graph models and graph algorithms were also deployed in the framework of multi-agent system techniques. Agents were placed in vertices (representing power sources or loads or distribution boards) and edges (representing connections) observing the working states of these elements. A spanning tree was picked to represent the distribution network being studied. A Fault Detection Algorithm (by means of tree-sorting) was also applied to locate circuit faults. Once a fault is identified, a Reconfiguration Algorithm (backed by mathematical programming) was applied to the graph model to simulate the negotiation process within the multi-agent system associated with the power network. The reconfiguration algorithm is used to help the associated multi-agent system achieve an optimal solution during the power network restoration process. This approach has been successfully deployed. Results can be found in References [2.14] and [2.15].

\subsection{Integrate MAS Technologies into a Prototype Laboratory System for Testing.}

Task 1 of this final report described the general approach for MAS communication system design. Task 2 describes the specific hardware acquired for first stage prototyping during the project.

The M52259DEMOKIT demonstration kit for Freescale's MCF52259 microcontroller includes the M52259DEMOMCU and M52259DEMOCOM boards. The MCF52259 32-bit device is based on the Version 2 ColdFire core operating at a frequency up to $80 \mathrm{MHz}$, offering high performance and low power consumption. On-chip memories connected tightly to the processor core include up to 512 Kbytes of flash memory and 64 Kbytes of static random access memory (SRAM). The M52259 can be programmed by using Freescale CODEWARRIOR Software.

M52259DEMOMCU Features:

- $\quad$ Fast Ethernet Controller (FEC)

- USB Physical Layer Interface (PHY)

- Mini-FlexBus External Bus Interface

- FlexCAN 2.0B Module

- One universal asynchronous/synchronous receiver/transmitters $\underline{\text { XBee/X-bee PRO module: }}$

The wireless communication hardware used is the XBee/X-bee PRO module operates within the ISM 2.4 GHz frequency band and can meet IEEE 802.15.4 standards. Two types of boards are present, one with a USB interface and two Xbee modules with RS-232 interfaces.

Wireless USB Adapters:

- USB 2.0 Interface

- High-Speed Data Rate of 54 Mbps

- $2.4 \mathrm{GHz}$-Compliant Equipment 


\section{Communication Modes:}

- Wired Modes: The microcontrollers communicate through Ethernet, twisted pair, or RS232 serial interfaces.

- Wireless Mode: The microcontrollers communicate, in accordance with IEEE Standard 802.11/15, through Xbee/Xbee PRO radio modules.

\subsection{Demonstrate Multi-Agent Concepts on a Laboratory-scale Power System Simulator}

The Lane Department of Computer Science and Electrical Engineering at West Virginia University maintains a hardware analog power simulator that was donated to the university and installed in the 1970s. The simulator, as shown in Figure 2.6.1, is a low power replica of a generation, transmission, and distribution system that contains commercial, industrial and residential loads. Power can be supplied to the loads from different circuits, and external generators can be connected to the simulator. Line switching is accomplished manually, and relays are electromechanical. Most of the loads are resistive. One of the loads is inductive. There is also a capacitor bank which can be manually connected to improve the voltage and power factor at the loads. This simulator is normally used as a teaching tool for undergraduate and graduate power system courses offered at WVU.

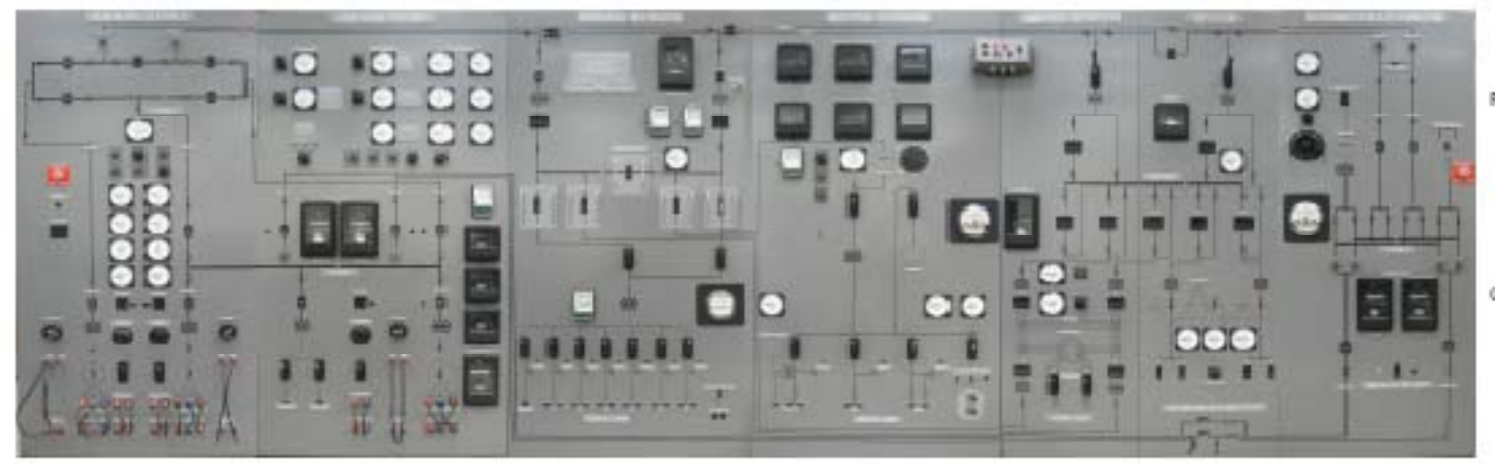

Figure 2.6.1. Photograph of the Original WVU Analog Simulator

The purpose of this activity was to retrofit the analog simulator to mimic (simulate) a microgrid of the future by installing digital relays, intelligent electronic devices and control agents, distributed energy resources (generation and storage), and a FACTS device.

The benefits of this upgrade would be substantial. For instance, students in undergraduate classes would get exposed to real world hardware before entering the workplace. Also, new hardware would be integrated with existing hardware (as is found in existing power systems) so the students would get to see the entire range of equipment used. In addition the system could then also be used for research into autonomous reconfiguration schemes by the graduate students doing research with APERC, a powerful capability not achievable with the present simulator.

More details about this effort are provided in Section D7.3 of Deliverable 7. 


\section{Task 3. \\ Modeling and Simulation.}

\subsection{Introduction.}

This task will leverage other research efforts already undertaken by Southern California Edison to identify and evaluate advanced distribution devices and practices that are expected to enable enhanced interoperability, and ultimately improve system performance and service to customers. Since SCE is participating in several US Department of Energy supported projects focused on grid modernization, this task will take the opportunity to become familiar with the equipment, protection devices, operating procedures, and communication and control protocols of SCE's anticipated next generation transmission and distribution system to the extent permissible by SCE.

\subsubsection{Subtasks of Task 3.}

This task has six subtasks as follows:

3.1. Conduct a literature search related to modeling and simulation of next generation electricity delivery systems to ascertain if similar research has been done or is currently underway,

3.2. Study advanced technologies being deployed and/or considered for evaluation by the SCE CoF to identify appropriate agents and corresponding attributes that enable optimal assimilation into the smart grid,

3.3. Consult with electric utilities to gather information and select commercially available power system analysis software or methodologies,

3.4. Become sufficiently familiar with the selected power system analysis software and T\&D system methodologies so as to evaluate the feasibility of interfacing MAS with associated software algorithms and simulation tools,

3.5. Integrate the agent algorithms and modeling techniques into the commercial modeling programs and simulation facilities, and

3.6. Adapt the multi-agent algorithms and models to these T\&D modeling software packages.

\subsubsection{Deliverables for Task 3.}

The only deliverable for this task, Deliverable 6, is a report on specific applications of software modeling and laboratory simulation for MAS in electric power systems.

\subsection{Ascertain from Literature Review if Similar Research Projects Offer Collaboration.}

The main function of an electrical power system is to maintain the availability of energy to all the connected loads. Aboard all the ships of the US Navy (especially the newer all-electric crafts), the power system's main function is to keep all the shipboard systems and equipment operational: it operates the hydraulic systems, powers the communication system, controls the weapons, radio, sonar, radar, etc. If the electric power is down the ship is defenseless against enemy attacks. When faults or damages occur as a result of a battle or components' failure, isolation of the faulted equipment by protective devices can lead to de-energization of critical 
loads, decreasing the ships ability to survive. Thus, it is essential to isolate just the faulty part and reconfigure the system in a rapid, reliable and autonomous manner to supply the maximum of critical loads.

At present, shipboard power system reconfiguration is mainly accomplished manually. Therefore, autonomous reconfiguration and restoration can increase the survivability and reliability of the ship by decreasing human intervention and providing a faster response. Civilian electric distribution systems exhibit similar characteristics. Several researchers have addressed different strategies to tackle the reconfiguration problem in power systems in general. The reconfiguration problem is combinatorial due to many switching alternatives that increase with the growing complexity of the system.

Several techniques, such as mathematical programming, heuristic and meta-heuristic techniques, have been proposed in the literature to solve this type of problem. Most of these approaches rely on centralized control and depend on a powerful computing and communication infrastructure to handle the huge amounts of data. A major drawback of the centralized system is its vulnerability to single point failures, especially in naval vessels where the central controller may be hit in battles. The solution to this problem is to distribute control. Multi-Agent System (MAS) technology is a branch of distributed intelligence that deals with concentrating information gathering and decision making at the device or component level. By making power system components more intelligent, the conventional centralized control methodologies are shifted to distributed and localized approaches thus reducing human intervention. In recent years, MAS technology has been increasingly applied in an attempt to solve power system reconfiguration problems. Some authors have presented the restoration of a distribution power system with a radial configuration. In their model, they have addressed load priority, limitations on the generating source, and transfer capacity. The agent model was developed in a JADE platform hosting different types of agents. Some authors have modeled the MAS as an Intelligent Power Router (IPR). These routers are deployed in a power distribution network to route the power the same way routers do in information distribution networks. Other authors proposed distributed MAS for power system restoration where they introduced Bus Agents to find a suboptimal target network configuration by interaction with a Facilitator Agent that acts as a manager in the decision making process.

In most of the work on power distribution system restoration and reconfiguration using MAS, the decision or the solution is found by relying on communication and negotiation of the various agents. These agents simply look for a path to restore the power without regard as to the effect of their actions on the distribution system's voltage profile. While it might work for relatively small radial networks (where no voltage violations or drops are expected) it is clear that for relatively large distribution systems, finding a feasible restoration path without considering possible voltage violations might not yield an acceptable solution. Agents should have a priori knowledge of the voltage profile(s) that could result from the reconfiguration actions they propose. In other words, the voltage profile plays an important role in choosing a reconfiguration scheme for power restoration. Furthermore, for a looped distribution system, it becomes very complex to find a target configuration relying on communication alone, since the power does not flow in only one direction as in a radial system.

In this Task, a multi-agent system was designed and deployed base on a hardware prototype of a shipboard power system for reconfiguration, restoration and load shedding. The designed MAS is based on the human Immune System (IS). It is able to detect and isolate faults, reconfigure and restore power to the healthy loads and perform load shedding when necessary. 
The MAS system was implemented using microcontrollers. Both simulation and experimental results were generated in the cases of faults at the feeder level and shortages in power supply. The results show that the proposed MAS is fast and very effective in solving the above mentioned problems, making MAS technologies a good candidate for solving complex problems in power systems in general and shipboard power systems in particular.

\subsection{Study Feeders Typical to CoF for Attributes and Agents to Assimilate in CoF.}

Figure 3.2.1 shows a main distribution feeder supplied from source " $\mathrm{S}$ " and protected by relays R1, R2 and R3. The basic philosophy here is, for maximum fault current in line 3 (which would be a fault at bus (4); the time of operation of relay R2 is made larger than time of operation of R3 at least by a time interval called "Coordination Time Interval (CTI)". CTI depends on factors like errors in current transformers, potential transformer and relays, and on circuit breaker opening time. In case of electromechanical relays, overshoot is also considered. Similarly, relay R2 and R1 are coordinated for a maximum fault at bus 3. The nature of inverse relay curves is such that once coordinated for maximum current, they will be coordinated for lower fault currents too. As clear from Figure 3.2.1, R2 will back up R3 and R1 will back up R2.

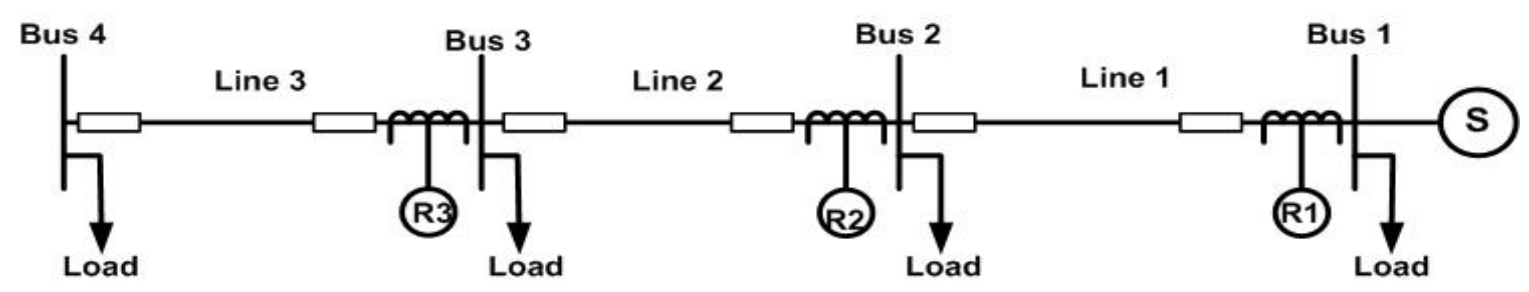

Figure 3.2.1. Feeder System Arrangement.

\subsubsection{DG Impact on Protection and Coordination.}

New generation capacity from distributed generation (DG) is growing in the overall generation mix partly due to state and national initiatives to address global-warming concerns. Installation of DGs in the distribution system increases the fault current throughout the system. Depending on the penetration level, location, and type of DGs, the protection system may lose coordination with high numbers of DG units since the protection system is now designed using conventional techniques. Circuit breaker capabilities and settings of protective relays that were previously designed for a system without DGs may not safely and appropriately coordinate to manage faults in a system with DGs. Accurate fault analysis in systems with inverter-based generation may not be possible with conventional fault analysis tools and techniques. At low inverter-based DG penetration levels (e.g., below five percent of system capacity), conventional protection analysis methods may be quite adequate. At high penetration levels (e.g., ten percent or higher), however, new methods may be necessary. See Figure 3.2.2.

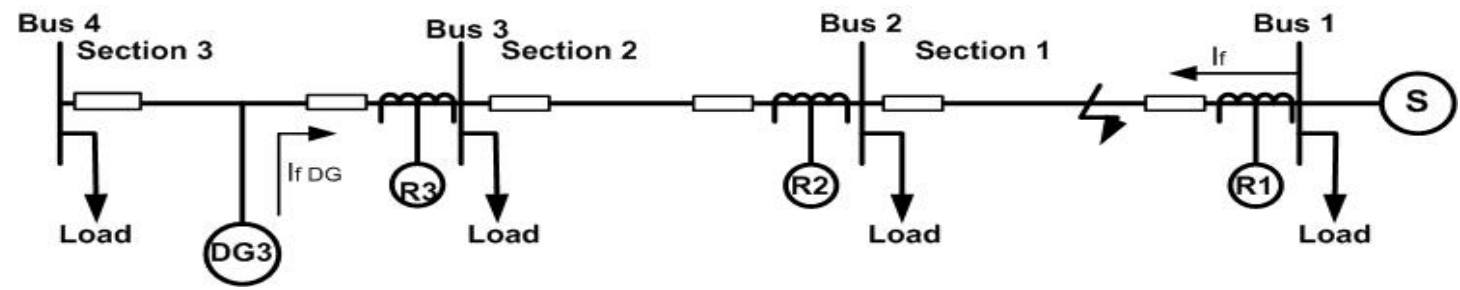

Figure 3.2.2. DG Fault Contribution to System. 
If only DG3 is connected, R2 and R3 will sense downstream current for faults in section 3 and upstream current for faults in section 1. It is important to note here that for any given fault downstream or upstream, these relays will sense the same fault current. This would create a conflict. Since it is wished to clear only the faulted section, R3 is required to operate before R2 for any fault in section 3, but require R2 to operate before R3 for a fault in section 1 . Since these relays sense the same current for either of these faults, it is impossible to achieve coordination with the existing scheme. See Figure 3.2.3.

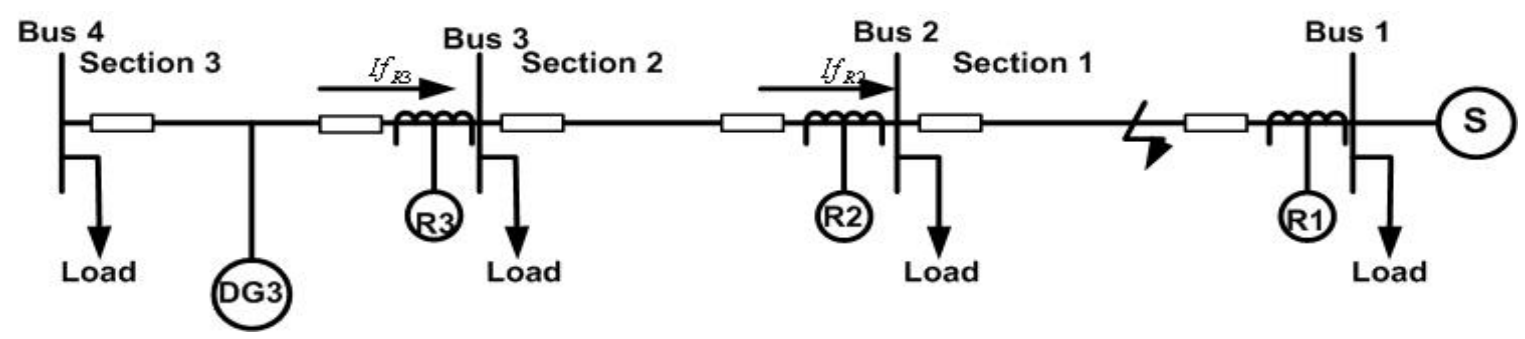

Figure 3.2.3. DG Impact on Protection and Coordination.

\subsubsection{MAS Structure.}

The proposed multi-agent protection coordination system, as shown in Figure 3.2.4, consists of relay agents, distributed generator agents and equipment agents. The agents can communicate with each other not only within the same agent society, but also within different agent societies.

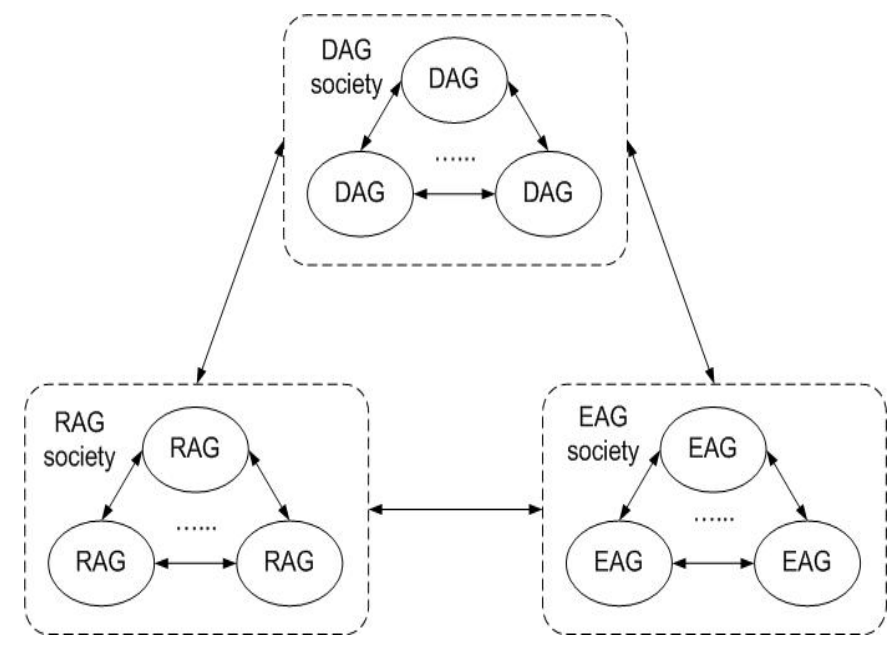

DAG: Distributed Generator Agent

RAG: Relay Agent

EAG: Equipment Agent

$\longleftrightarrow$ : Communication

Figure 3.2.4. Multi-Agent Architecture for Peer-to-Peer Relay Coordination.

A group of agents that achieve the same relay function can form a small society, such as the overcurrent relay agent society and the differential relay agent society. Each group defines its own relay roles and roles define the relay logic associated with them. When an agent joins a society, it takes up one or more relay roles and acquires the relay logic of that role. 
The proposed architecture uses geographically distributed agents located in a number of Intelligent Electronic Devices. An IED is a hardware environment that has the necessary computational, communication, and other I/O capabilities needed to support a software agent. The agent takes sensory input, which might include local measurements of the current, voltage, and breaker status, from system, and produces output actions such as breaker trip signals, adjusting transformer tap settings, and switching signals in capacitor banks. See Figure 3.2.5 and Figure 3.2.6.

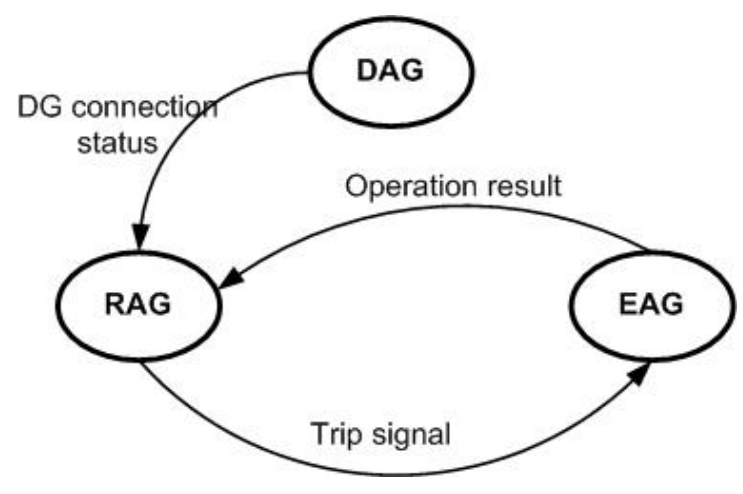

Figure 3.2.5. Agent Communication for Protection.

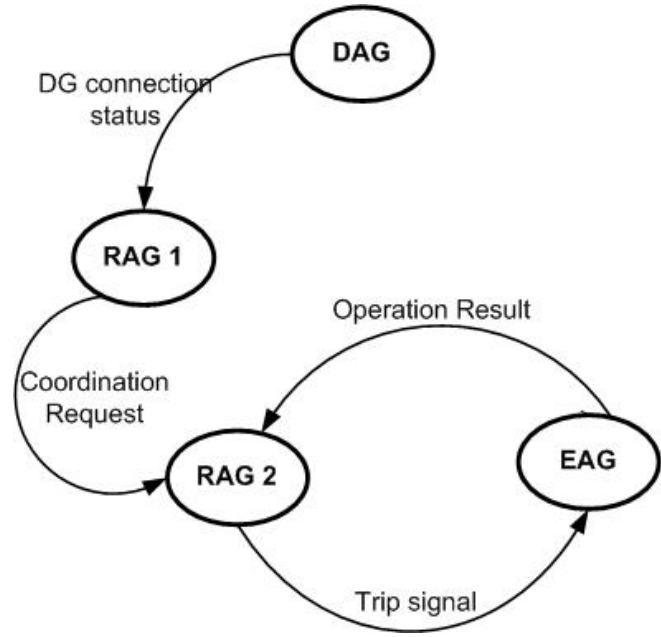

Figure 3.2.6. Agent Communication for Protection and Coordination.

\subsubsection{Agents.}

\section{- Relay Agent.}

Each relay installed in the system will be regarded as one relay agent. The relay agent structure is illustrated in Figure 3.2.4. The relay agent searches for relevant information by communicating with other agents. Its purpose is to detect relay misoperations, breaker failures and DG connection status and perform backup protection with much better performance that can be expected from traditional methods. Coordination strategy will be different under different circumstances. It should also be emphasized that, since the communication is available, relay strategy is the main methodology for the relay function and coordination. See Figure 3.2.7.

\section{- Distributed Generator Agent.}

Distributed generator agent takes every single distributed generator as one agent. In protection coordination, distributed generator agent mainly communicates with relay agent in the distribution system to provide connection status of its own for the relay agent to coordinate.

\section{- Equipment Agent.}

The equipment agent includes CT agent, breaker agent, etc. These distributed equipment collects local power system information, operates the local power system equipment, and communicate information with relay agent to provide protection and coordination function. 


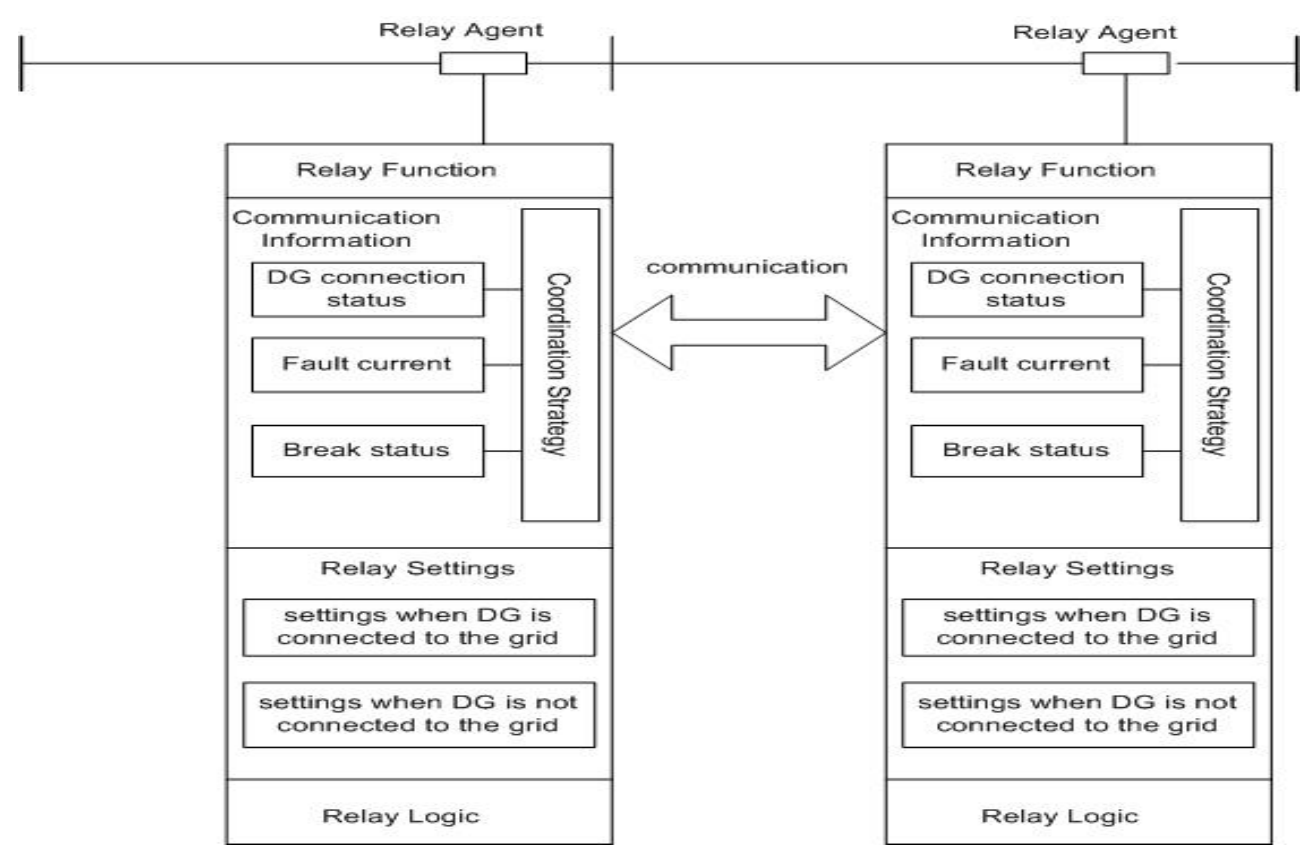

Figure 3.2.7. Structure of Relay Agent Coordination.

\subsubsection{Simulation.}

\section{- EMTP/ATP and JADE Solution}

Most functions of digital relays are realized by the program code of a microprocessor. The large number of functions and complexity of digital relays program requires the tools to increasingly verify and test the algorithms.

The developed relay algorithms have been tested with waveform signals generated by the non-real-time simulator like PSCAD/EMTDC, this approach has the disadvantage that they cannot be tested dynamically. Some researchers have used the real-time simulation like RTDS (Real time digital simulator) to test the relay algorithm dynamically, but, this method needs additional hardware which is rather expensive. Methods if model interface are classified as playback or open-loop simulation, and real-time or closed-loop simulation. Open loop simulation means that it is impossible to feed any trip signal from the relay under the simulation process. On the other hand, closed loop simulation is made by running the simulation in realtime and therefore any trip signal is provided by the relay under the simulation process. EMTP MODELS is a PC-based closed loop simulation tool, simulation can be tested dynamically, and also the cost is low. This simulation tool was not used in the project.

\section{- EMTP MODELS}

EMTP, the ElectroMagnetic Transient Program, is widely used in the electric utility industry. But it can be used only in the static state of the power system or in the preset sequential state. The system diagram of EMTP operation is shown in Figure 3.2.8. The EMTP is used as a signal generator for the settings of the relays. It is called the open-loop (also called play-back simulation). 
EMTP/ATP

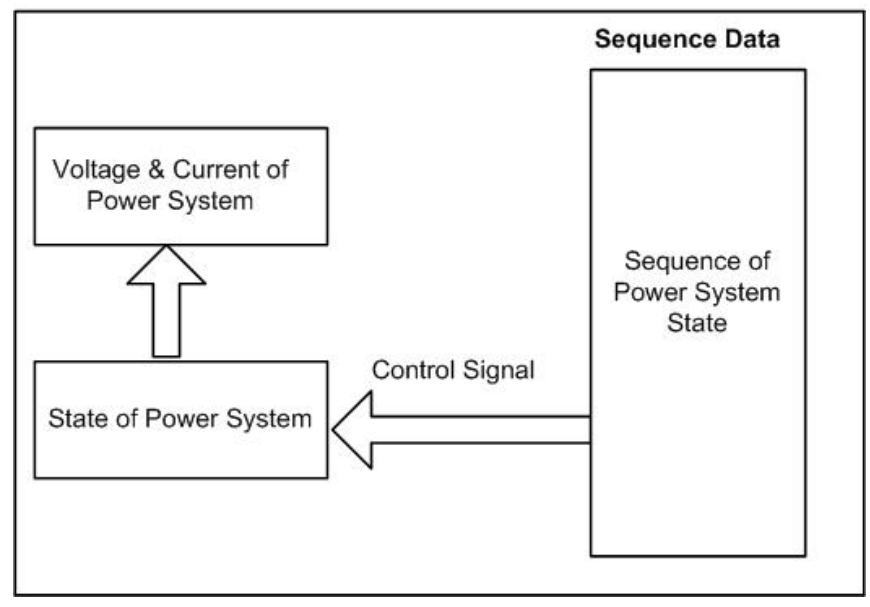

Figure 3.2.8. EMTP Operation without MODELS.

The development of MODELS has made it possible to control component characteristics in an external program having voltages and currents from the circuit as input. MODELS is a symbolic language interpreter for the EMTP that has recently gained popularity for the electromagnetic transient's phenomenon modeling. The MODELS provides the monitoring the controllability of power systems as well as some other algebraic and relational operations for programming. MODELS is able to change dynamically the states of power system in response to the simulated results of EMTP power system. The system diagram of EMTP MODELS operation is shown in Figure 3.2.9. The voltage signals and the current signals of EMTP power system enter the input of MODELS. These signals are processed by the program coded with MODELS and the output signals by MODELS change the states of EMTP power system. This process is repeated until the simulation is completed. MODELS makes it possible to simulate the interaction between the power system and the measuring or control system. It is called the closed-loop simulation.

EMTPIATP

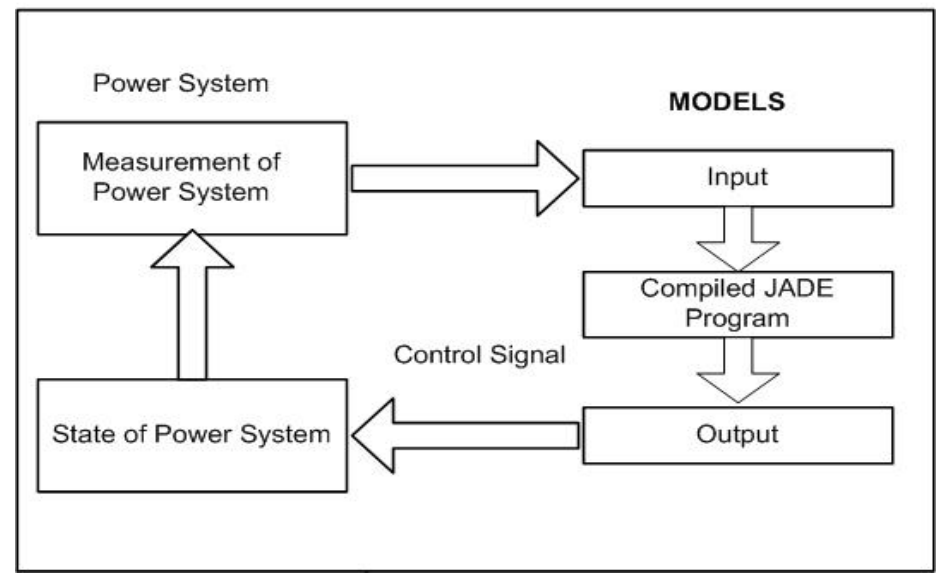

Figure 3.2.9. System Diagram of MAS Protection and Coordination by MODELS. 
The signals of the node voltage values and the node current values are sent to the inputs of MODELS and the parameter settings are used in the algorithm of MODELS. If the fault is detected and the trip of circuit breaker is decided by the algorithm, then MODELS sends the opening command of the EMTP switch to the EMTP main simulation. The states of the power systems, the operating state of the internal algorithm, and the state of the inputs and outputs of the relay are recorded in the computer file during the simulation. After the simulation, all the states can be analyzed with the recorded file minutely.

\section{- InterPSS and JADE Solution}

The other simulation solution is to implement power system in Java language and simply integrating it with JADE as a simple agent. InterPSS is an open source development project aimed to develop an Internet technology based software system for design, analysis and simulation of power system. The project is based on the latest Internet technology, including Java, XML, the Eclipse platform and other open-source software system. Its system architecture allows components developed by others to be easily plugged into InterPSS and allows its components to be integrated into other systems. The power system simulation environment can be constructed by integrating Inter PASS engines in JADE agents. The communication between agents and power system can be carried out just by message passing capabilities of JADE.

\section{- InterPSS Power System Simulation Framework .}

InterPSS system with JADE architecture is shown in the Figure 3.2.10. At the center of the architecture is the power system simulation framework, which is based on an object-oriented power system simulation model. UML (Unified Model Language) is used to model power systems for simulation purpose and Eclipse EMF to generate source code for the simulation framework. The goal of InterPSS design is to be flexible and extensible software system. Since it is an open-source system, worked largely by volunteers, ease of maintenance is also an important part of the design goal.

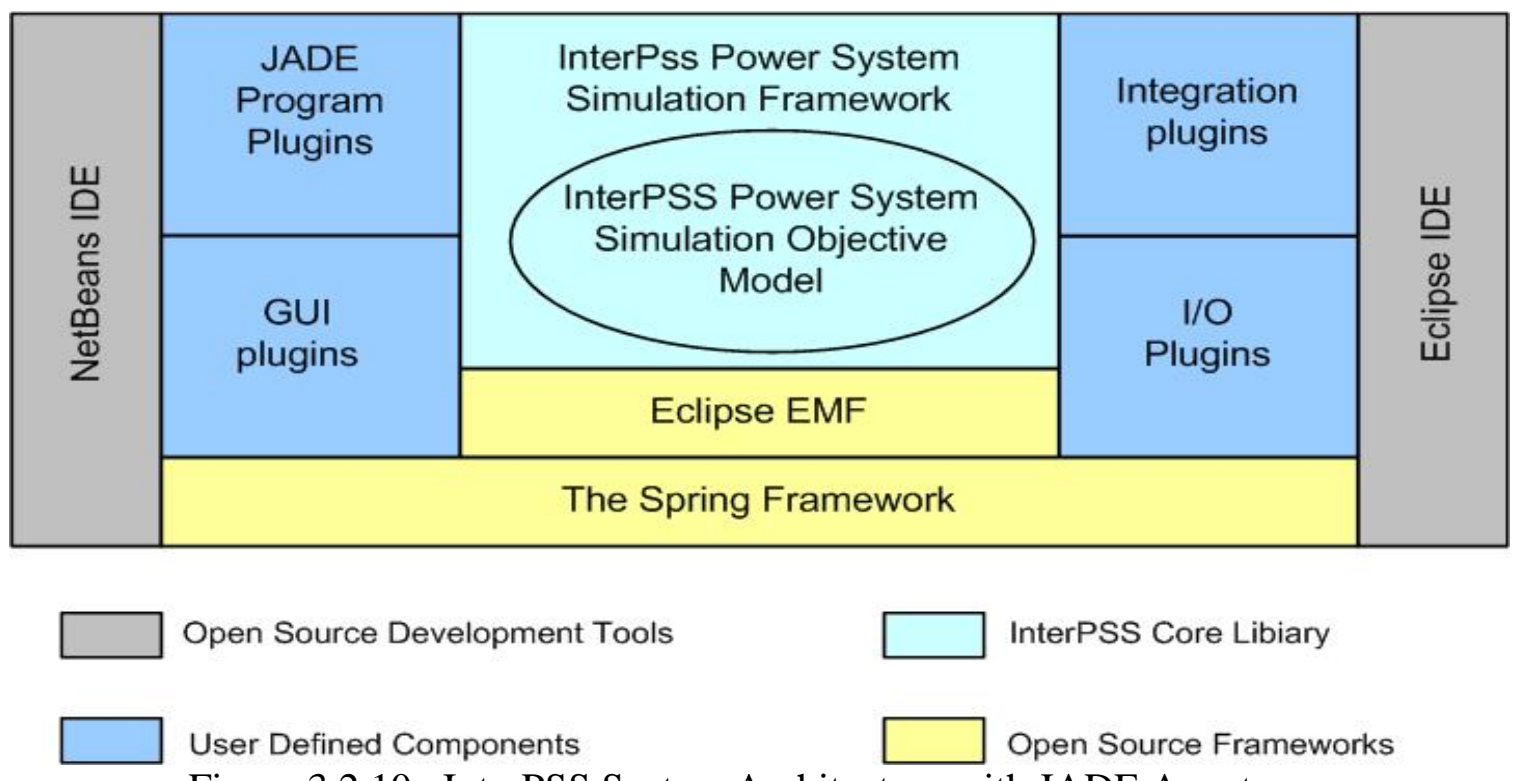

Figure 3.2.10. InterPSS System Architecture with JADE Agent. 
User GUI, I/O modules, and integration interface implementations are plug-ins, which can be easily customized and replaced. Our JADE agent will be these kinds of plug-ins. InterPSS components are configured and/or integrated based on the Spring Framework. InterPSS development team uses Eclipse IDE (Integrated Development Environment) and NetBeans IDE to do the development.

\section{- InterPSS Power System Simulation Framework}

InterPSS foundation is a power system simulation framework, which consists of a power system simulation object model and a set of interfaces with which the outside world can interact with InterPSS core simulation engine. The framework is designed in a loosely coupled way that the core simulation engine could be easily extended or integrated into other systems as a standalone component.

Traditionally, a power system simulation program has a fixed format for creating data files as the input. InterPSS core simulation engine publishes its Java API. InterPSS does not a fixed format for the input. Instead, InterPSS provides a set of plug-ins, called input File Adapters, for inputting data in particular format into InterPSS.

\section{- InterPSS Object Model}

InterPSS simulation framework consists of a set of simulation objects, and interfaces with which the outside world could interact with these objects. SimuContext is the interface.

\subsubsection{Network Topology.}

Modern power system simulation algorithms are mostly built on the power network nodal admittance Y-matrix. From the simulation perspective, power system network topology could be described as a Network object containing a set of connected Bus objects and Branch objects. InterPSS uses the following network topology object model to describe power system networks for the simulation purpose.

A Bus object is a "node" to which a set of Branch objects could be connected. A Branch object is an "edge" with two terminals (from side and to side) which can be connected between two Bus objects (fromBus and toBus). A Network object is a container where Bus objects can be defined, and Branch objects can be connected between these Bus objects to form a network topology for simulation purposes.

\section{- Network Classes}

InterPSS breaks the power simulation problem into many relative simple concept layers, such as Network, AclfNetwork, etc. InterPSS uses object-oriented programming techniques, such as "Inheritance" and "Composition", to reuse functionality defined in other concept layers. Currently, the following concept layers are defined. More concept layers for such concepts as harmonics and OPF-optimal power flow will be added into InterPSS object model in the future.

\section{- Bus Classes.}

InterPSS uses Bus object concept to describe logical power system buses for the simulation purpose. 


\section{- Branch Classes.}

InterPSS uses Branch object concept to describe logical power system branches for the simulation purpose. A Branch object can connect to a From-Bus object and a To-Bus object.

\subsubsection{Switch/Breaker Model.}

In power system control centers, power network structure change information are gathered in terms of actual switch/break control actions. InterPSS currently supports two ways to model the switch/breaker control actions.

\section{- JADE Agent Simulation Framework}

This Framework has been discussed previously in Section 3.2.5.

\section{- InterPSS Application Integration}

InterPSS provides a graphic editor for a user to draw one-line diagram and enter study case data. Currently it has basic functions to create a simple power system one-line diagram, input data, perform analysis and generate analysis reports. The one-line diagram and data can be saved into a file and then reloaded into the editor for further analysis. InterPSS can integrate InterPSS components/modules, such as InterPSS simulation core engine, into other applications, or integrate other open-source libraries InterPSS as to augment its functionality and integrate other applications as independent runtime components with InterPSS.

InterPSS Custom Run Scripts, Java Code, are used to integrate other applications. InterPSS simulation engine is running outside of Graphic Editor as an independent application. The outside applications can be integrated by following two key steps: use a custom adapter to export InterPSS object model information to a text file, and use IpssJavaExec.exec( ) to launch an outside application using the generated text file as the input. The application's output is then picked up by InterPSS for display.

\subsection{Select through Consults with Utilities a Program to Analyze the CoF Grid.}

This subtask was not able to be effectively performed because the project was constrained in the amount and level of commitment that SCE project utility persons could give to this subtask, which resulted in limited contacts and consults with other utilities.

\subsection{Adapt NETL Capabilities to Enhance the Current CoF Capabilities.}

This subtask was not able to be effectively performed because the project personnel at NETL were limited (due to other NETL obligations) in the amount of commitment they could give to this subtask. However, an alternative effort was pursued to adapt certain other capabilities to enhance the CoF capabilities as described below.

Evolution of the electric power industry due to recent restructuring and deregulation has led to a major paradigm shift from centralized systems towards decentralized solutions. Considering power system reconfiguration in particular, centralized solutions based on artificial neural networks, a genetic algorithm, and expert systems have been proposed. However, such solutions do not adequately address the requirements of modern power systems. Efficient monitoring, control, restoration, and reconfiguration of power systems require distributed decentralized 
control. Multi-agent systems have been applied for solving problems such as reconfiguration, restoration, fault identification, diagnosis, and power system protection.

Most of the work employing multi-agent systems for power system reconfiguration and restoration use an agent to represent an electric component such as buses, switches, and circuit breakers, etc. A major problem with such solutions is the limited application especially for large scale power systems with hundreds of components. The size of the multi-agent system used in addressing the problem and interactions among agents introduce challenges due to scalability. Another issue that becomes more and more important as the size of target system increases is that of communication overhead. Communication and coordination policies used in a multiagent system can have serious impact on scalability. However, improvement can be achieved by optimizing the size of agent communities and their organizational patterns.

A major challenge of agent architectures for large scale power systems is scalability. Most existing architectures associate an agent with an electronic component in the system. The idea is to have agents with local information communicate with each other to reach a solution. However, the amount of communication, coordination, and other resource requirements for such solutions will soon outweigh the anticipated benefits of decentralization. So, no matter how promising the suggested multi-agent system is, its size will always pose a problem.

This triggers the need to find a trade-off between decentralization and size of the multi-agent system. Efficient solutions would place a bound on the number of agents in the system. Clustering techniques have been developed to identify logical power system partitions that agents can monitor. These dedicated clusters or partitions have been developed rather than employ an agent to monitor each node or device. This reduces the resources utilized and the complexity of the system. Our proposed approach significantly reduces the number of agents in the system and the communication overhead while minimizing the system complexity and computational overhead.

Furthermore, in the event of a fault, a decentralized MAS architecture has been proposed so that the affected nodes are quickly identified for reconfiguration. The agents in this decentralized MAS use only local information, and the MAS is topology-independent. The decentralized MAS is used as a baseline to compare the performance of the proposed scalable MAS and the time taken to reconfigure a distribution feeder in the event a fault is detected.

\subsubsection{Proposed Topology-Independent Decentralized MAS Architecture.}

The proposed topology-independent MAS uses three types of agents. Bus Agent (BA) represents buses in the power system.

Processor Agent (PA) represents a common place in the multi-agent system where information from other agents is accumulated. Switch Agent (SA) monitors and controls switches in the power system and performs the task of turning a switch on or off. The proposed topologyindependent decentralized MAS was applied to the Circuit of the Future (COF) system, developed by Southern California Edison (SCE).

There are a total of 66 buses and 7 switches in the system requiring 66 bus agents and 7 switch agents. The coordination of different tasks by all the agents involved in fault identification and reconfiguration is described in Algorithm I. Figure 3.4.1 is a graphical representation of the Circuit of the Future $(\mathrm{CoF})$. Fault scenarios and the resulting message flow have also been depicted. 


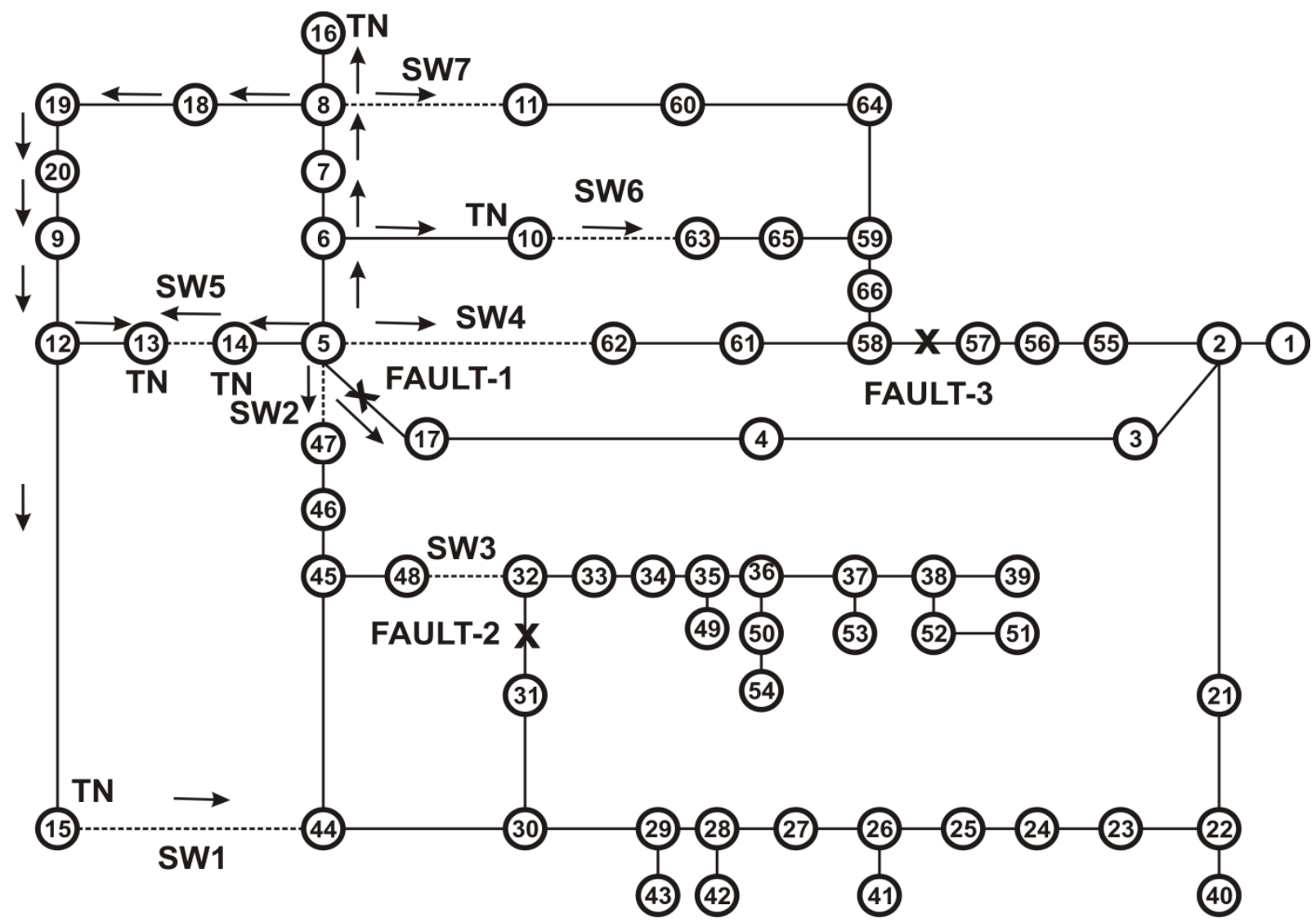

Figure 3.4.1. Graphical representation of the Circuit of the Future and Example Fault Scenarios.

\subsubsection{Spectral Clustering Techniques for Power System Partitioning.}

In this section, a spectral clustering algorithm is proposed to logically partition the power network into clusters of connected buses. In the algorithm, a clustering parameter is selected that represents the notion of electric distances. To tune the clustering algorithm for partitioning a power system and to give importance to electrical properties of the system, the bus impedance matrix is used to acquire the necessary information for clustering.

Agents are then assigned to each cluster or partition. This modified approach scales well as the size of the power system increases by reducing both the total number of agents in the system and the communication overhead. However, to gain these benefits, the agents in the clusters acquire additional knowledge about the topology in their cluster.

First, a matrix representation of the large scale structure is constructed. Eigenvalues and eigenvectors of the matrix are calculated. These eigenvalues and vectors provide global information about the structure of the matrix and its connectivity. Also, a mapping of data points to a lower dimensional representation is performed based on one or more eigenvectors. Finally, the data points are assigned to different clusters. The spectral clustering algorithm is tailored for partitioning a power system. Let $\mathrm{S}$ represent the set of buses in the power system. Then, after obtaining the adjacency matrix, the $\mathrm{N} \times \mathrm{N}$ diagonal degree matrix is obtained using the spectral clustering algorithm which uses the normalized Laplacian matrix defined as 


$$
\begin{gathered}
N=|S| \\
A-\left[a_{i j}\right] \quad \text { is the } \mathrm{N} \times \mathrm{N} \text { adjacency matrix } \\
Z=\left[Z b u s_{i j}\right] \\
a_{i j}=\frac{1}{a b s\left(z_{i j}\right)} \\
D(i, j)=\sum_{j=1}^{N} A(i, j) \\
\hat{\mathrm{A}}=D^{-\left(\frac{1}{2}\right)} A D^{-\left(\frac{1}{2}\right)}
\end{gathered}
$$

\section{Algorithm I}

(Function and coordination of agents after a fault is detected between Bus i and Bus j)

\section{Bus Agent}

BAj communicates with neighbors to disseminate fault information An agent BAx receives a message and performs the following tasks:

IF BAx has power and a neighboring switch

THEN Inform sender that BAx is energized

\section{ELSE}

IF BAx has neighboring switch THEN

Check if bus at other end of switch is energized

IF bus is energized THEN

Suggest switch position to PA

ENDIF

\section{ENDIF}

IF BAx is at terminal node THEN

Send an intermediate list of agents affected by a fault to PA ELSE

Add self to the list of agents affected by the fault

FOR all elements in the list of neighbors

Send the list generated in previous step

\section{ENDFOR}

ENDIF

\section{Processor Agent}

Determines all agents affected by the fault. Determines all potential switches for reconfiguration. Selects a switch for reconfiguration 


\section{Switch Agent}

Performs the final task of changing the switch status

The proposed spectral clustering algorithm was extended to Southern California Edison's Circuit of the Future. There are a total of 66 buses in this system. The eigenvectors and eigenvalues of the Laplacian matrix are calculated. From these eigenvectors the $66 \times 5$ matrix $V$ is constructed, where $v_{1}, v_{2}, v_{3}, v_{4}$ and $v_{5}$ are the five largest eigenvectors of A . The structure of the eigenvectors of the Laplacian matrix encodes the structure of the resulting clusters. Table 3.4.2 summarizes the details of the five clusters in the CoF after applying the proposed spectral clustering algorithm.

Table 3.4.2. Results obtained after Applying Spectral Clustering on Circuit of the Future.

\begin{tabular}{|c|c|c|}
\hline $\begin{array}{c}\text { Cluster } \\
\text { No. }\end{array}$ & $\begin{array}{c}\text { Total Bus } \\
\text { Count }\end{array}$ & Buses in the Cluster \\
\hline Cluster 1 & 22 & $\begin{array}{c}1,2,3,55,56,57,58,61,62,66,59,65,63,64,60, \\
11,21,22,40,23,24,25\end{array}$ \\
\hline Cluster 2 & 16 & $4,17,5,6,14,7,10,8,16,18,19,20,9,12,13,15$ \\
\hline Cluster 3 & 13 & $47,46,45,44,48,30,29,28,43,42,27,26,41$ \\
\hline Cluster 4 & 6 & $31,32,33,34,35,49$ \\
\hline Cluster 5 & 9 & $36,50,54,37,53,38,52,39,51$ \\
\hline
\end{tabular}

\subsubsection{Proposed Scalable Multi-Agent System Architecture.}

In this section, a scalable multi-agent architecture using fewer agents is described. The MAS architecture uses two types of agents.

Cluster Agent is assigned to each cluster or partition instead of assigning an agent to each bus as proposed in the decentralized topology-independent MAS. This results in a smaller agent system. These agents are described as cluster agents or CAs. Figure 3.4.2 shows the 5 cluster agents CA1-CA5 and 7 reconfiguration switches, SW1-SW7. These cluster agents can communicate with each other and work in coordination to perform different functions. By dividing the large system into logical clusters and assigning these to different agents, the size and complexity of the problem are reduced for each agent, thereby making the load flow calculations, monitoring, reconfiguration, or restoration efficient and manageable. Switch Agent turns a reconfiguration switch on or off based on the chosen path and given constraints.

The overall algorithm describing the interaction between agents in the proposed scalable MAS is described in Algorithm II. 


\begin{tabular}{|ll|}
\hline & \multicolumn{1}{c|}{ Algorithm II } \\
Step 1: & Obtain a weighted graph $\mathrm{G}(\mathrm{V}, \mathrm{E})$ from the power system \\
Step 2: & Apply spectral clustering algorithm to obtain $\mathrm{K}$ partitions \\
Step 3: & $\begin{array}{c}\text { Assign agents to clusters obtained Interaction of cluster agent } C A_{\mathrm{j}} \text { with a faulty } \\
\text { cluster agent } C A_{\mathrm{i}}\end{array}$ \\
Step 4: & $C A_{\mathrm{j}}$ sends potential source nodes and associated costs to $C A_{\mathrm{i}}$ For a cluster agent \\
& with fault \\
Step 4a: & Dynamically update model of cluster topology \\
Step 5: & $C A_{\mathrm{i}}$ communicates with its neighboring cluster agents for finding a solution \\
Step 6: & $C A_{\mathrm{i}}$ determines nodes affected by the fault \\
Step 7: & $C A_{\mathrm{i}}$ performs analysis of each alternate source node $\mathrm{Si}$ in parallel \\
Step 8: & $C A_{\mathrm{i}}$ evaluates potential reconfiguration solutions from neighbors \\
Step 9: & $C A_{\mathrm{i}}$ determines a feasible best solution based on constraints \\
Step 10: & $C A_{\mathrm{i}}$ communicates with selected switch agent to perform the final reconfiguration \\
\hline
\end{tabular}

As an example, it is assumed there is a fault between Bus 7 and Bus 8, in the cluster assigned to CA2 as shown in Figure 3.4.2. When the fault is detected, CA2 communicates with its neighbors CA1 and CA3, to initiate the task of identifying possible reconfigurable paths and computing their associated costs. CA2 determines the nodes affected by the fault. The affected nodes are highlighted in gray in Figure 3.4.2.

Cluster 2

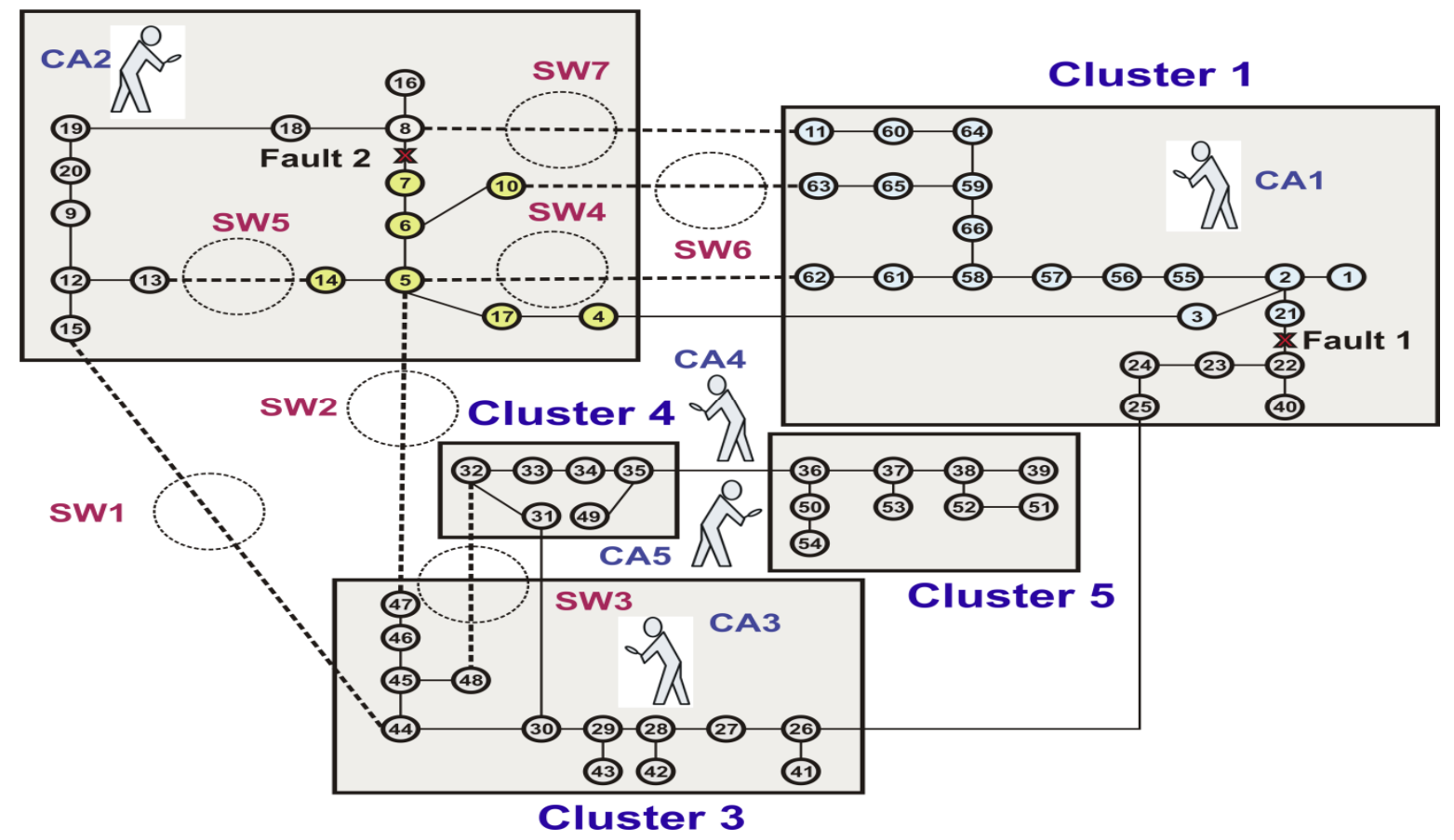

Figure 3.4.2. Logically Clustered Circuit of the Future and Example Fault Scenarios.

The neighboring agents perform their calculations to suggest alternate routes and associated costs to CA2. CA2 determines the feasibility of different source nodes. Notice in this case that switching on SW2, SW4, or SW6 cannot solve the problem since they will not be able to supply 
power to the affected nodes. So CA2 rules these out. The potential alternate source nodes are identified as Node 11 in Cluster 1, and Node 44 in Cluster 3. The third option involves internal switching within Cluster 2 between Nodes 13 and 14. Out of these options the agent selects the one with the minimum cost. All possible single and three-phase faults are simulated for SCE's Circuit of the Future.

\subsubsection{Simulation Results and Discussions.}

The Circuit of the Future was used to simulate all possible single and three-phase faults for both the topology-independent decentralized MAS architecture and the scalable MAS architecture. The results obtained from these simulations are shown in Figures 3.4.3, 3.4.4 and 3.4.5.

Figure 3.4.3 presents a comparison of the two MAS approaches in terms of the communication overhead. Communication overhead is defined as the total messages passed in the agent community to reach a decision. It can be clearly seen in the figure that the scalable MAS experiences a far less communication overhead to reach the same decision as compared to the completely decentralized MAS.

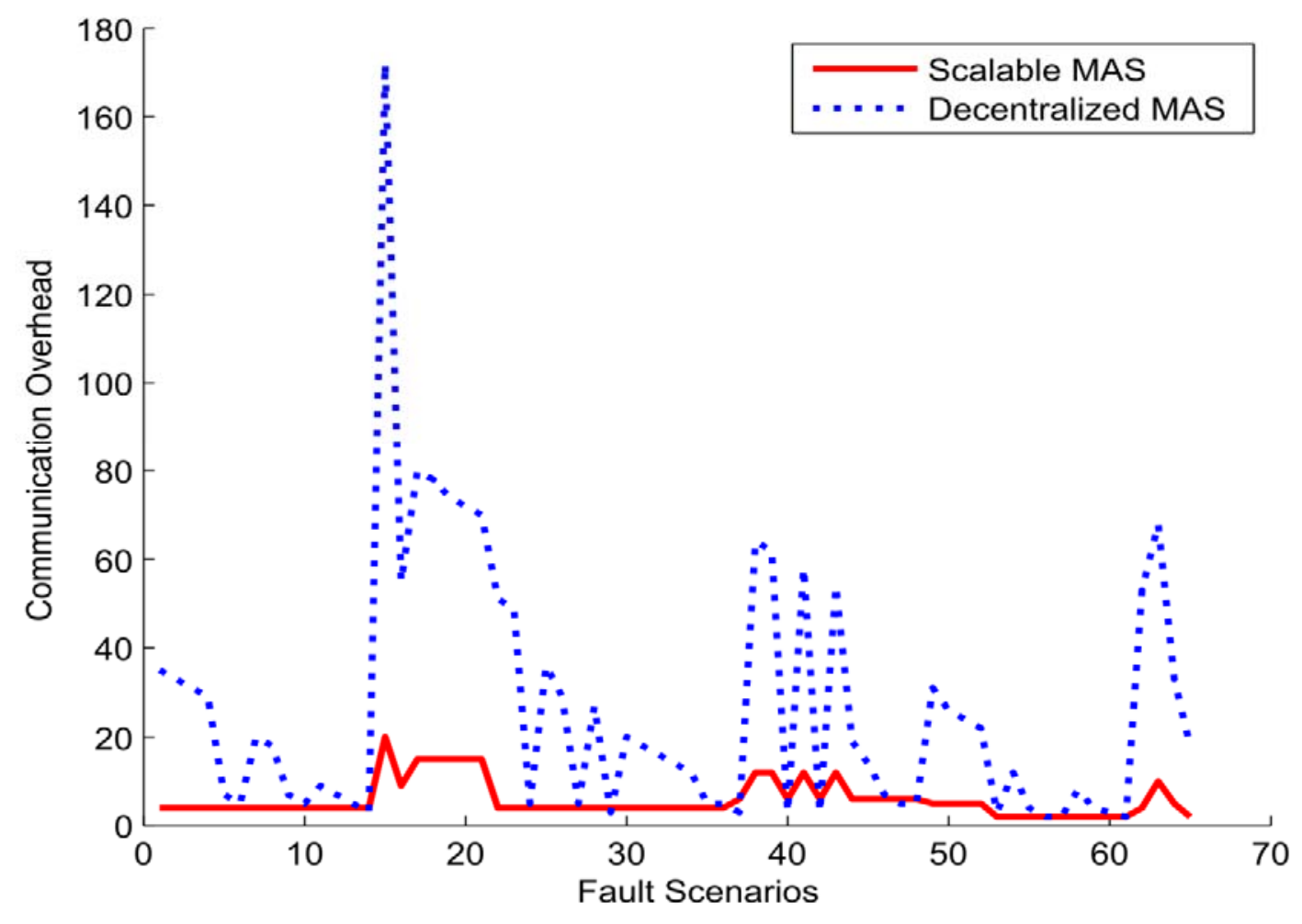

Figure 3.4.3. Comparison of Communication Overhead.

Next, the two approaches are compared in terms of the computational overhead. For the purpose of these simulations, the computational overhead is defined as the total time taken by the MAS to arrive at a decision to perform reconfiguration in the event of a fault. Multiple simulations of all possible fault scenarios were performed to obtain a comprehensive profile of the total time taken by each MAS. The results are shown in Figure 3.4.4. It can be seen that the scalable MAS takes less total time to reach a decision. 


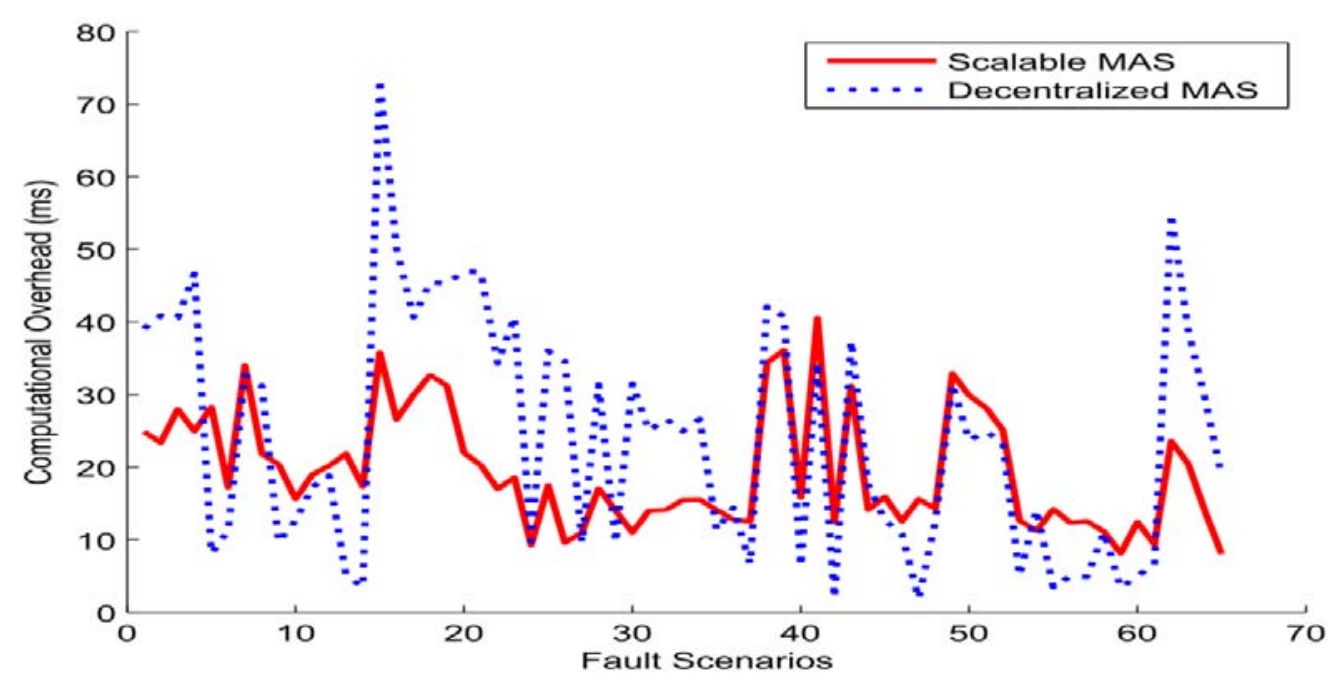

Figure 3.4.4. Comparison of Computational Overhead.

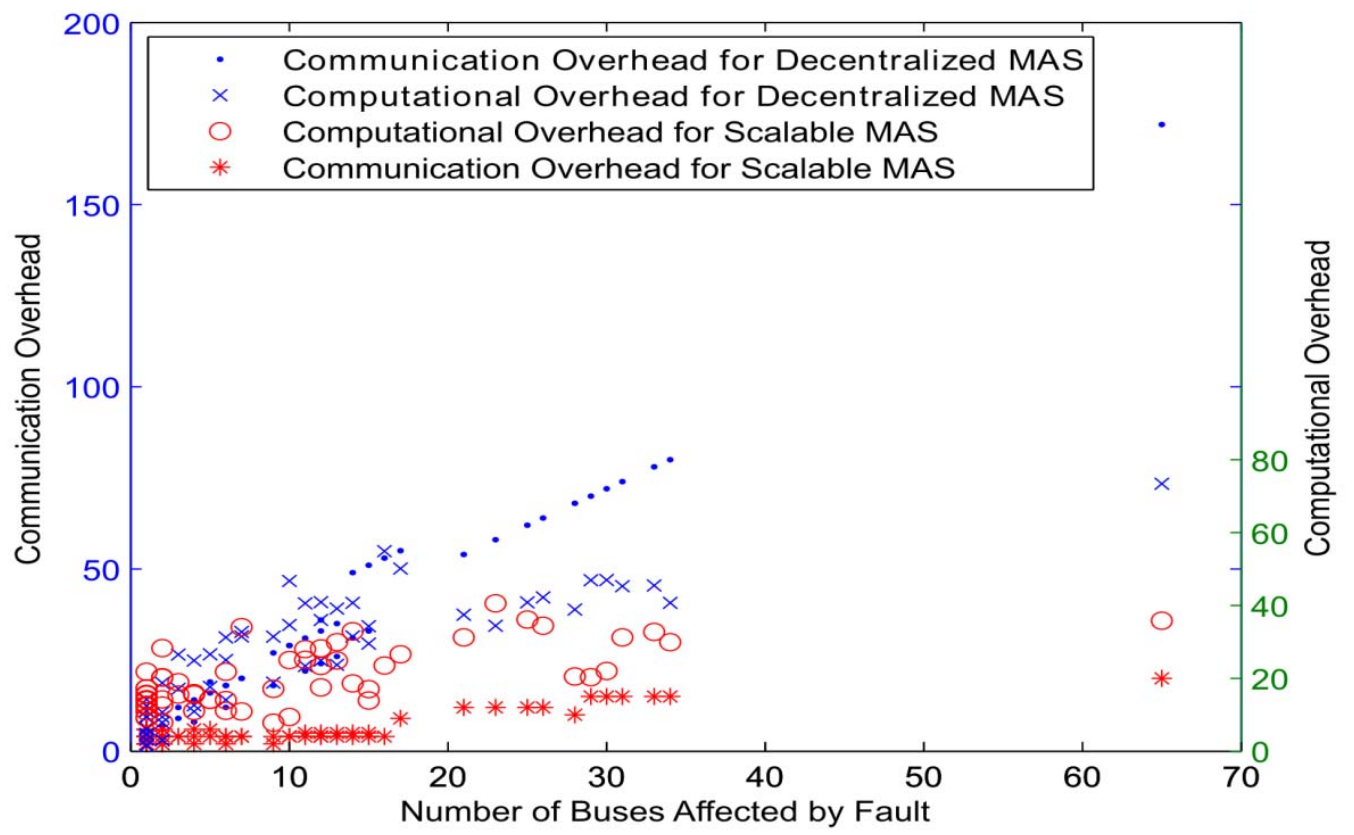

Figure 3.4.5. Comparison of Performance with Respect to Buses Affected by a Fault.

Finally, the relationship is investigated between the total number of buses affected by a fault and the performance for each of the proposed approaches. The observations are summarized in Figure 3.4.5. When the number of buses affected by a fault is small the scalable approach does incur some overhead. However, when the number of buses affected by a fault increases, the proposed scalable MAS outperforms the topology-independent decentralized MAS. 


\section{Task 4. Economic Analysis and Evaluation.}

\subsection{Introduction.}

In general, it is anticipated that MAS deployment expense can be minimized and economic benefit maximized through implementation strategies that integrate agent functions within various DG, DER and DSM technologies. The MAS's ability to utilize assets more efficiently and effectively may delay or postpone capital infrastructure investments that would otherwise be required by the utility. The economics of the MAS may be further enhanced by the relative easy and low cost integration of its functionalities and software into the control and communication modules already present for digital signal processor (DSP) controlled DSM and inverter-based DG/DER technologies.

\subsubsection{Subtasks for Task 4.}

This task has four subtasks as follows:

4.1. To evaluate the economic feasibility of incorporating MAS into specific transmission and distribution (T\&D) system protection, control and communications devices/components and DG/DER/DSM components as well as to identify obstacles to deployment,

4.2. To perform economic analysis to assess the social welfare and the commercial viability for various deployment strategies and technical performance levels for MAS at the T\&D levels,

4.3. To utilize input from the electric utility to develop case studies to assess industry and national economic benefits attributed to the MAS, and

4.4. To prepare a summary report for the economic analyses and evaluation of the CoF.

\subsubsection{Deliverables for Task 4}

The one deliverable for Task 4, Deliverable 2, consists of two parts. Deliverable 2a is a report about the economic evaluation of increasing the intelligence levels (using MAS) for electric power systems. Deliverable $2 b$ is a report about the economic feasibility of MAS for electric power systems.

\subsection{Evaluate Broad-Based Economic Analysis of Incorporating MAS in CoF Grid.}

Grid reliability has been an issue of major concern in the United States since the August 2003 Northeast blackout. Despite the millions of dollars spent annually on maintenance and capital improvements by utilities the country's electrical infrastructure is still over stressed and antiquated. The integral components that make up the electric grid are aging rapidly because they are being used in ways that increase equipment stress resulting in accelerated wear and unexpected equipment failures. This stress makes the whole system more susceptible to critical failures. Loads are at risk due to systems that deliver power long distances from large centralized power generation over an aging infrastructure. This infrastructure lacks dynamic system re-configuration and distributed resources that could improve the resiliency and reliability of the system. When outages do occur on the distribution systems, they must be located and assessed by sending crews into the field in response to customer calls. An extended 
period of time is often required to identify the failure and restore customer service. Significant savings as well as improved reliability and resiliency could be acquired by simply reducing the restoration times by employing automated fault detection and isolation.

The technical issues surrounding reliability and resiliency are addressed in earlier sections of this report. Although technical solutions may exist, their employment by utilities depends on their economic feasibility and the inherent risk associated with capital investments of this nature. A global survey conducted by Newton-Evans Research in the mid 1990's revealed that the most significant obstacle to the implementation of circuit automation, with the exception of lack of funds, was an economic justification. This remains the major impediment to wide scale deployment of smart grid technologies. It is difficult to provide economic justification for these investments solely on direct benefits because the direct benefits derived from automation are rarely sufficient to cover all of the associated costs. The inability to provide a sound business case may be attributed to the fact that typical cost benefit analyses conducted on these types of projects often overlook indirect benefits which may be more difficult to identify and quantify. To address this problem, a broad-based economic analysis factoring in the social costs and benefits is employed to assess the true economic cost of automating a distribution circuit and implementing agents. Policy changes that may be needed to entice public utilities to make the capital investments of this nature are also discussed.

The economics and risks associated with the automation of a distribution circuit are examined here in the broader context of a socioeconomic analysis to provide insight into the likelihood of large scale adoption of distribution automation. The analysis is based on a case study of Southern California Edison's Circuit of the Future. SCE spent considerable time and effort in examining hardware upgrades to reduce the stress and improve the power quality on this circuit in an effort to avoid building a new substation. It was determined by the company that the best course of action would be to install three new inter-point isolation switches, upgrade the remaining switches, purchase a mobile 2.5 MVA mobile DG and install a 6,200 KVAR D-SVC unit. In addition to these upgrades, APERC proposed investigating the installation of multifunctional agents to enhance the reliability and resiliency of the circuit and to facilitate the automation process.

\subsubsection{Economic Feasibility of Incorporating MAS in CoF.}

\section{- Potential Benefits}

Potential benefits that may be derived from the modernization of the CoF were identified and attempts were made to assign quantitative values to them. The list of benefits includes both direct benefits and indirect benefits which are often difficult to identify and quantify. The departure of key SCE researchers assigned to the project and SCE withdrawal after their departure precludes the calculation of each specific benefit and the actual total benefits. Therefore the benefits discussion is forced to rely on general inferences and results of similar studies to augment the benefit calculations when actual data was not available.

Table 4.1 contains a list of potential benefits and the associated beneficiaries. The automation of distribution feeders has a number of indirect benefits associated with it that can affect other enterprise activities making it difficult to allocate their contribution to specific functions or activities. Therefore no attempts have been made to allocate benefits or assign values to specific activities. Benefits are instead calculated and classified by beneficiary. 
Table 4.1 Potential CoF Benefits

\begin{tabular}{|c|c|c|c|}
\hline Beneficiary & Type of Benefit & Explanation & Category \\
\hline \multirow[t]{4}{*}{ Consumer } & C\&I Interruption Prevention & Avoided cost of interruptions & Reliability \\
\hline & $\begin{array}{l}\text { Residential Interruption } \\
\text { Prevention }\end{array}$ & Avoided cost of interruptions & Reliability \\
\hline & C\&I PQ Improvement & $\begin{array}{l}\text { PQ is compounded with } \\
\text { interruptions }\end{array}$ & Reliability \\
\hline & Reduce Price of Electricity & Peak shaving and load flattening & Economic \\
\hline \multirow[t]{8}{*}{ Utility } & $\begin{array}{l}\text { Operational Response } \\
\text { Reduction }\end{array}$ & $\begin{array}{l}\text { Avoided cost of interruption } \\
\text { response }\end{array}$ & Reliability \\
\hline & Equipment Wear \& Tear & $\begin{array}{l}\text { Less equipment wear due to fewer } \\
\text { interruptions }\end{array}$ & Reliability \\
\hline & Increased Sales & Meters turn instead of interrupted & Reliability \\
\hline & Reduce Congestion Costs & $\begin{array}{l}\text { Using DA / DG resources mitigates } \\
\text { congestion }\end{array}$ & Economic \\
\hline & $\begin{array}{c}\text { Cost } \\
\text { Avoidance/Utilization/Ratings }\end{array}$ & $\begin{array}{c}\text { Distribution transformer \& dynamic } \\
\text { ratings }\end{array}$ & Economic \\
\hline & Asset Life Extension & $\begin{array}{c}\text { Extend life of transformers by not } \\
\text { overloading }\end{array}$ & Efficiency \\
\hline & Reduce Operating Staff & $\begin{array}{l}\text { Staff reduced through SA \& IT } \\
\text { Integration }\end{array}$ & Efficiency \\
\hline & Worker Injuries and Claims & Reducing fatal and non-fatal Injuries & Safety \\
\hline \multirow[t]{6}{*}{ Society } & Reduce Blackout Probability & DA Islands avoid cost of blackout & Security \\
\hline & $\begin{array}{c}\text { National Security Benefits from } \\
\text { Energy Efficiency }\end{array}$ & $\begin{array}{l}\text { Reducing energy is valued as } \\
\text { reducing oil }\end{array}$ & Security \\
\hline & Reduce Price of Electricity & Peak shaving and load flattening & Economic \\
\hline & $\begin{array}{l}\text { Economic Development/Job } \\
\text { Creation }\end{array}$ & $\begin{array}{c}\text { Communications } / \mathrm{PQ} / \text { reliability } \\
\text { grows jobs }\end{array}$ & Economic \\
\hline & $\begin{array}{l}\text { Emission Reduction } \\
\left(\mathrm{NO}_{\mathrm{x}}, \mathrm{SO}_{\mathrm{x}} \& \mathrm{CO}_{2}\right)\end{array}$ & DA resources reduce emissions & Environmental \\
\hline & Public Health and Safety & Life support customers/ traffic safety & Safety \\
\hline
\end{tabular}

Studies suggest that the most predominant benefit is quality of service improvement measured by the reduction of SAIDI/customer minutes lost. See References [4.1] and [4.2]. Achieving satisfactory SAIDI levels is crucial to maintaining customer loyalty. Studies have shown that even partial automation can deliver up to a $25 \%$ improvement in increased customer 
satisfaction. Feeder automation has been shown to deliver flexibility and reliability in excess of the planned benefits. Studies indicate that customer minutes lost may be reduced by $33 \%$ on distribution circuits through automation alone. See References [4.3] and [4.4. The reduction may increase significantly when distributed generation and multi-agents are incorporated. The reduction of lost minuets with these technologies employed may be more in the order of $80 \%$ assuming a 60 percent reduction in power outages and a 10\% DG penetration on the circuit. For the CoF this translates into an average annual benefit of $\$ 5.6$ million based on the cost of power outages study for the state. See Reference [4.5]. Under the current tariff structure in place in California these benefits would accrue to the consumers as illustrated in Table 4.2.

Table 4.2 CoF Annual Benefits Estimates

\begin{tabular}{|l|r|r|}
\hline Beneficiary & \multicolumn{2}{|c|}{ Benefit Estimate (\$1000) } \\
\hline Consumer & 5,482 & \\
\hline Savings for circuit & 97 & \\
\hline PQ savings & 2 & \\
\hline Residential savings & & 5,581 \\
\hline Total Consumer & & \\
\hline Utility & 149 & \\
\hline Avoided revenue losses & 155 & \\
\hline Savings from avoided new substation construction & 25 & \\
\hline Savings reduction in congestion & & 323 \\
\hline Total Utility & & \\
\hline Society & & 2,423 \\
\hline Total Societal & & 8,327 \\
\hline Total Benefits & & \\
\hline
\end{tabular}

The benefits that accrue to the utility are calculated based on the loss of revenues associated with the outages on the circuit, reductions in congestion costs and saving from deferred substation construction. These benefits are estimated to be approximately $\$ 323,000$. Additional utility benefits that may be associated with reduced outage times (such as reductions in work related injuries and reductions in operations staff) were not calculated due to a lack of data. Studies suggest that these benefits account for less than one percent of the total operational benefits when estimated for a utility or a state with multiple utilities. Thus, they are negligible for a distribution circuit and would have virtually no impact on the results of the analysis.

The societal benefits were estimated based on the economic gains that would be obtainable with a more reliable power source. This was accomplished by multiplying the C\&I benefits by the complex multiplier of 1.5 to account for the linkages that these sectors have throughout the state's economy. While this method can account for benefits associated with reduction in power outages and economic development/job creation, it fails to account for benefits derived from national security that results from improved energy efficiency, public health and safety or emission reduction ( $\mathrm{NOx}, \mathrm{SOx} \& \mathrm{CO}_{2}$ ). The benefits derived from lower electricity prices are also excluded because an estimate of the level of price reduction was not obtainable as a result of 
the inability to construct case studies with utility input. ${ }^{3}$ The inclusion of these benefits would have resulted in a higher level of social benefits. Thus, the estimated social benefit level of \$2.4 million is somewhat conservative.

\section{- Cost Estimates}

An overall cost estimate was constructed for the CoF based on incremental modifications made to automate the circuit and employ the agents. The individual cost elements are provided in Table 4.3.

Table 4.3 Cost Estimates

\begin{tabular}{|c|c|c|c|}
\hline Item & Quantity & Unit Cost \$ & Total \$ \\
\hline \multicolumn{4}{|l|}{ Circuit Upgrade Hardware } \\
\hline Mobile DG 2.5 MVA Capacity & 1 & 400,000 & 400,000 \\
\hline Inter-point Isolation Switches & 3 & 140,000 & 420,000 \\
\hline Upgrade Existing Switches & 1 & 40,000 & 40,000 \\
\hline SVC & 1 & 250,000 & 250,000 \\
\hline Total Hardware & & & $1,110,000$ \\
\hline \multicolumn{4}{|l|}{ Multi-Agent System } \\
\hline \multicolumn{4}{|l|}{ Software Development } \\
\hline Software V1.0 & 1 & 320,000 & 320,000 \\
\hline Software Upgrades & 1 & 150,000 & 150,000 \\
\hline Total Agent Software & & & 470,000 \\
\hline \multicolumn{4}{|l|}{ Agent Hardware } \\
\hline PC & 1 & 1,500 & 1,500 \\
\hline Agents & 40 & 4,00 & 16,000 \\
\hline CT's & 4 & 2,000 & 8,000 \\
\hline PT's & 4 & 2,000 & 8,000 \\
\hline WiMAX Clients & 10 & 9,00 & 9,000 \\
\hline Wi-Fi Clients & 40 & 500 & 20,000 \\
\hline Base Station & & & 75,000 \\
\hline Misc. Network Materials \& Installation & & & 50,000 \\
\hline Total Agent Hardware & & & 187,500 \\
\hline Total Multi-Agent System & & & 657,500 \\
\hline Total Cost & & & $\$ 1,767,500$ \\
\hline
\end{tabular}

The cost elements listed in Table 4.3 include installation cost. However, because the analysis is based on a circuit upgrade, all costs are assumed to be incremental costs and do not include any operating and maintenance $(\mathrm{O} \& \mathrm{M})$ costs or capital costs that would have otherwise been incurred. Any costs that may be displaced or deferred because of the circuit modification were included as a cost avoidance benefit attributed to CoF and were addressed in the benefits section.

The cost of adding the MAS to the system is approximately one-half the cost of the hardware investments proposed for the CoF. A significant portion of the MAS costs are associated with the development of the agent software and future software upgrades. These costs are estimated

${ }^{3}$ See Section 4.3 for a more detailed discussion of the measures preventing the development of these case studies. 
to be $\$ 470,000$ and account for approximately two-thirds of the total agent cost. The software development costs for the initial agent software are calculated by multiplying the fully load labor rates by the number of hours allocated to software development. The upgrade costs are assumed to be less than the original development cost due to decreases in the learning curve and the assumption that the original code will be modified not recreated. These costs are estimated to be approximately $\$ 150,000$.

The agent hardware costs are comprised of communications hardware and the hardware components of the agents themselves. The communications cost are calculated based on the assumption that a mesh network will be employed using a combination of Wi-Fi and WiMAX clients for communication between the agents, and between the agents and the substation as illustrated in Figure 4.1. However these costs could be eliminated if some form of high speed communications were already in place.

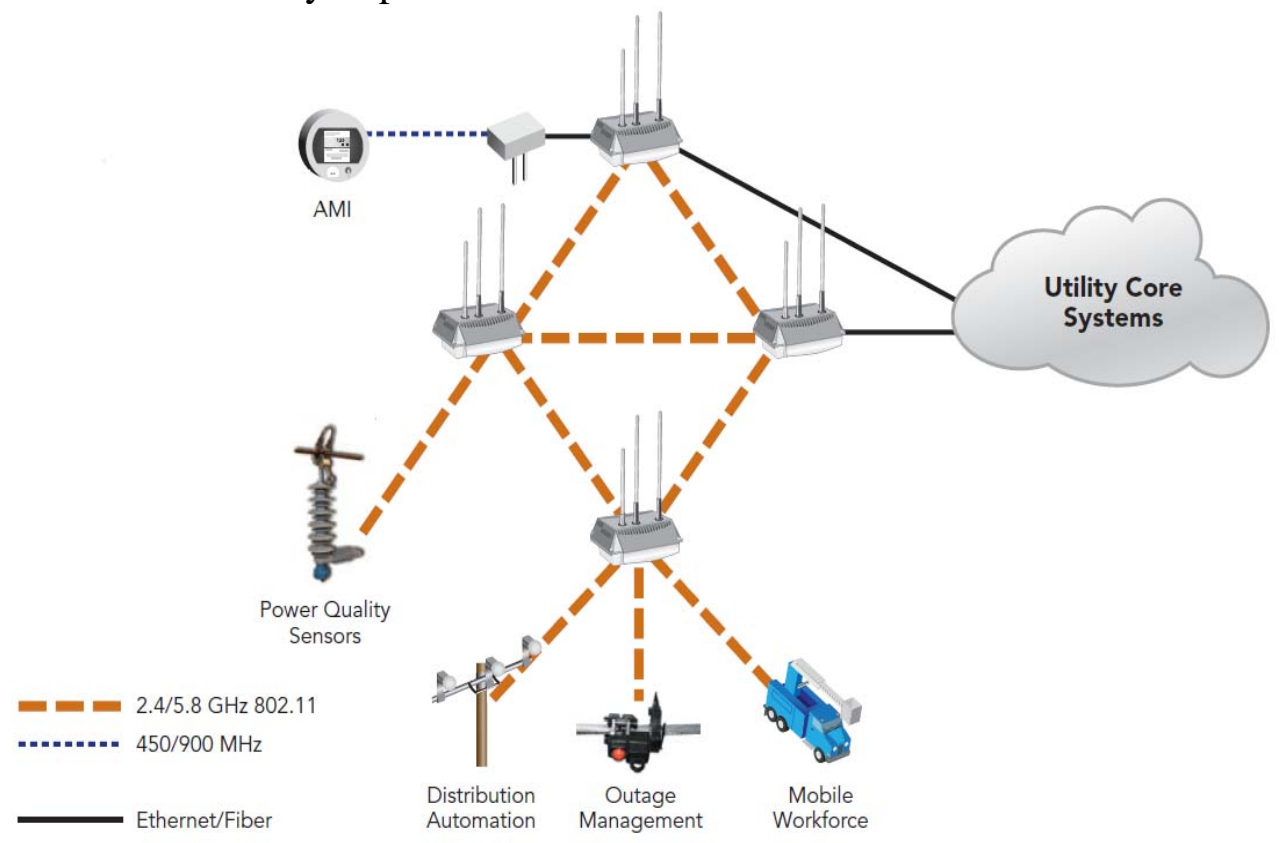

Figure 4.1 MAS Communications Network

\subsection{Assess Benefit Cost Analysis of Deploying MAS in SCE CoF Grid.}

As indicated in Table 4.3 the economic feasibility of implementing the MAS is marginal as indicated by the cost benefit ratio of 1.2. The ratio improves significantly to 21.2 when the consumer benefits are factored into the analysis.

Table 4.4 Benefit Cost Analysis

\begin{tabular}{|l|c|c|c|}
\hline Beneficiary & Benefit Estimate $\$ 1000$ & Cost Estimate $\$ 1000$ & Benefit/Cost Ratio \\
\hline Consumer & $\$ 37,452$ & $\$ 1,768$ & 21.2 \\
\hline Society & $\$ 25,412$ & $\$ 1,768$ & 9.2 \\
\hline Utility & $\$ 2,167$ & $\$ 1,768$ & 1.2 \\
\hline Total & $\$ 65,300$ & $\$ 1,768$ & 31.6 \\
\hline \multicolumn{2}{|c|}{ Benefits estimate based on PV of annual benefits @ 8\% for 10 yrs. } \\
\hline
\end{tabular}


However, as previously mentioned, these benefits cannot be captured by the utility under the current tariff structure. Given the questionable economics based on the utility's numbers it is unlikely this type of investment would take place. Therefore, in order for utilities to implement MAS, systemic revisions would need to be made to the tariff structure to provide recovery to utilities for investments of this nature. Once again, caution is warranted given the uncertainty in data estimates.

The economics look even more favorable when all benefits are factored into the analysis and the benefits to cost ratio increases to approximately 32. Although on the surface this appears to make the MAS even more attractive, it presents a new set of issues regarding the non-market aspects of some of the societal benefits. This refers to aspects of the MAS that do not produce a financial return, but are perceived by the public as being benefits. They can also be called "nonmarket benefits". They include benefits enjoyed by users and those valued by the general population. These benefits can be extremely difficult to capture and would also require some form of rate restructuring or government incentive.

The business case developed in this study demonstrates the need for the utility to be able to capture at least a portion of the societal and/or the consumer benefits to ensure that the investment is economically feasible given the overshadowing market uncertainty. It is unlikely that sufficient amounts of capital investment in distribution infrastructure will take place to meet rising reliability concerns under current market conditions. Although some investments in grid modernization to enhance reliability are being made by the electricity industry fundamental market failures make it difficult for utilities to recoup these investments. Therefore, regulatory intervention and/or financial incentives may be required to counter these market failures and promote wide scale modernization and reliability investments, or consumers must be willing to pay for improvements in power quality and reliability.

\subsection{Develop Case Studies using Utility Input to Estimate Benefits of MAS on CoF.}

It appears that the MAS is economically feasible given the cost benefit ratio for the utility is 1.2. However, with the degree of uncertainty in the data and the inherent market risks, this perception may be inaccurate. Therefore, before a definitive answer can be given, a comparison of restoration times for the MAS and conventional restoration practices should be conducted to validate the benefits of the preemptive and restorative properties of the MAS. It was the intent of the case studies to provide this validation. However, the departure of SCE from the project prevented this validation procedure and the development of more accurate societal benefits.

\subsection{Document SCE Support for Economic Feasibility and Benefit Analysis through Asset Planning.}

Evaluation of the quantities and types of circuit automation equipment to be installed on the CoF was performed by the engineers at SCE as part of their asset planning process. Subsequently, decisions to automate the substation were made to defer the capital investment associated with enlarging the substation or building a new substation. While SCE provided the cost estimates for the automation upgrades and reliability data for use in performing the economic assessment, their premature withdrawal from the project hindered the cost benefit analysis.

A detailed discussion of the economic analysis and associated results are provided in the topical report for Deliverable 2. 


\section{Project Deliverables}

The matrix shown in Figure D.1 provides an overview of how the seven project deliverables enabled the three main project focuses (multiple agents, communication schemes and business aspects) to yield the three main project results (improved protection, better reconfiguration and enhanced reliability) for electric power systems. Specific details are provided on the next pages.

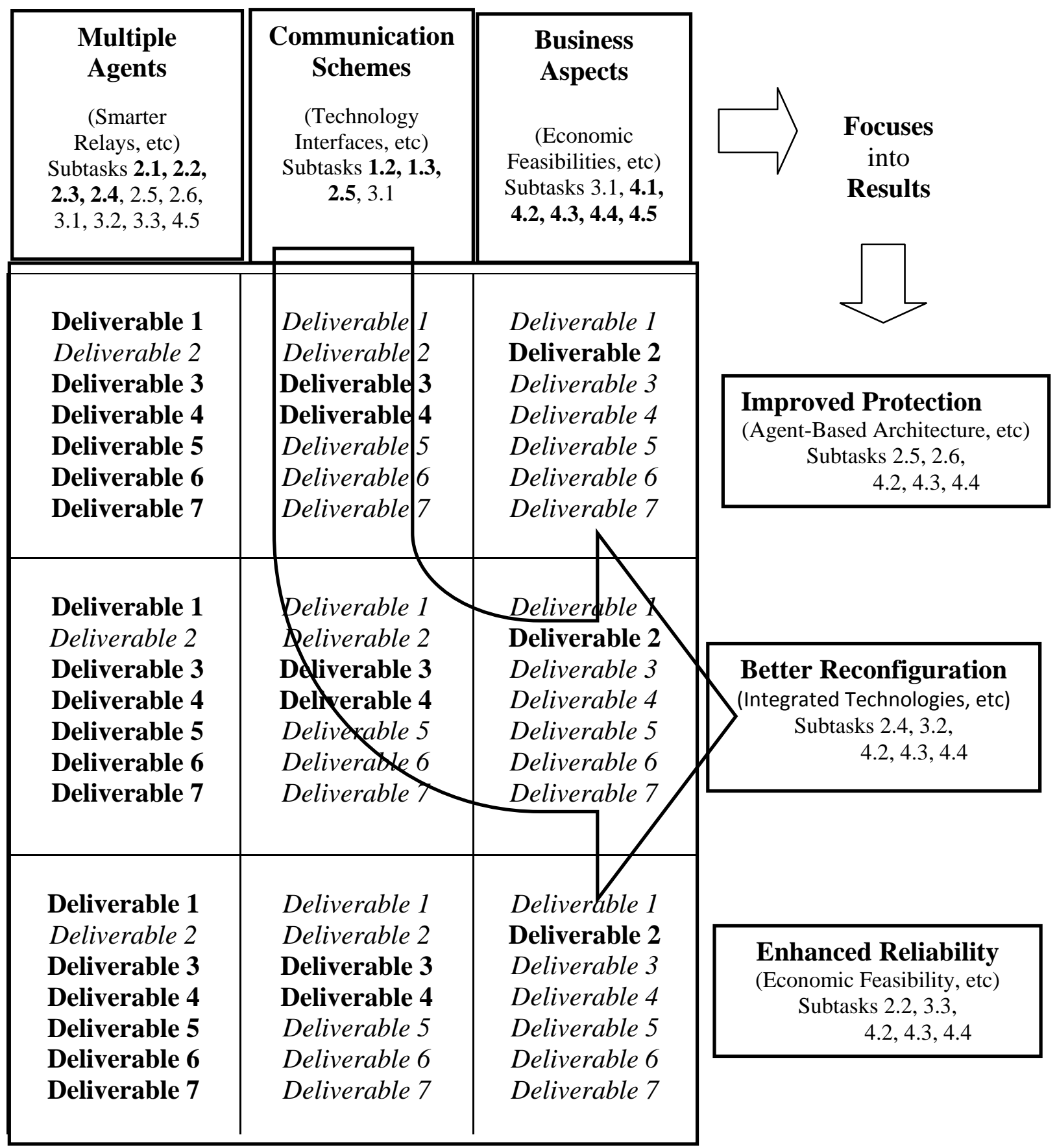

Figure D.1. Visualization of Relationships of Project Deliverables to Improved Protection, Reconfiguration and Reliability. 


\section{Deliverable 1. Report on Technical Requirements for a Multi-Agent System.}

\section{D1.0 Introduction.}

Power distribution systems are going through major automation changes due to increasing penetration of Distributed Energy Resources (DERs), Advanced Metering Infrastructures (AMIs), various power electronic control devices such as Static Var Compensators (SVCs) and smart switches. Added to these technological advances are the choices in power quality and prices that are becoming available to electricity (and energy) consumers. In the event of faults, power companies strive to resolve the system with as little disruption as possible. This results in improving the standard reliability indices such as SAIDI and SAIFI. Therefore, it is essential to isolate the faults and restore power as fast as possible by reconfiguring the electrical circuit.

Circuit restoration is a combinatorial problem due to the varied assortment of available switching operations, which increase with the growing complexity of the system. Several techniques, such as mathematical programming, heuristic and meta-heuristic techniques, have been proposed in the literature to solve this type of problem. Most of these approaches are centralized and depend on a powerful computing and communication infrastructure to handle the huge amount of data. In this era of deregulation, it is difficult to solve this problem by using conventional and centralized techniques since it requires a coordination of different parties such generation companies, consumers and independent system operators. Furthermore, the incorporation of DERs in the grid has added more complication to the system. The main reason for using DER is that it serves as a local generation when there is a power supply shortage. However, adding and interconnecting DERs to the power network gives rise to system stability problems and new protection coordination schemes must be developed.

This report on the technical requirements for a multi-agent immune system approach for power distribution systems is based on the paper titled "Multi-Agent Design for Power Distribution System Reconfiguration Based on the Artificial Immune System Algorithm” by Rabie Belkacemi and Ali Feliachi.

\section{D1.1 System Agent-based Intelligence.}

The Multi-Agent System (MAS) technology is a branch of distributed intelligence that deals with concentrating information gathering and decision making at the device or component level. By making the components of power systems intelligent, we shift from the conventional centralized control approaches to distributed and localized control thus reducing the human intervention. For example, by making a DG intelligent, which means that it has a knowledge capability, it can autonomously decide whether or not to connect to the grid. Such a decision is based on communication with other intelligent entities connected to the generation side or transmission side, and having key information about the state of the system (like voltage profile or stability).

While multi-agents have been applied to several areas of power systems, such as voltage stability, electricity market pricing, protection coordination, little research has been done on power system reconfiguration and restoration. Authors have presented the restoration of a distribution power system with a radial configuration. In their model, they have addressed load 
priority, limitations on the generating source, and transfer capacity. The agent model was developed in a JADE platform hosting different types of agents. Some authors have modeled the MAS as an Intelligent Power Router (IPR). These routers are deployed in a power distribution network to route the power the same way routers do in information distribution networks. Some authors proposed a distributed MAS for power system restoration, where they introduce Bus Agents to find a suboptimal target network configuration by interaction of these agents with a Facilitator Agent that acts as a manager in the decision making process. In most of the work on power distribution system restoration and reconfiguration using MAS, the decision or the solution is found by relying on communication and negotiation of the agents. The Agents simply look for a path to restore the power without regard as to the effect of their actions on the voltage profile. While it might work for relatively small radial networks (where no voltage violation or drop is expected), it is clear that for relatively large distribution systems, finding a path without considering possible voltage violations is not a complete solution. Agents should have a priori knowledge of the voltage profile resulting from the reconfiguration they propose. In other words, the voltage profile plays an important role in choosing a target configuration for power restoration. Furthermore, if the system is not radial it becomes very complex to find a target configuration relying on communication alone since the power does not flow in one direction only like in a radial system.

\section{D1.2 Human Immune System Technique.}

In recent work (and in this project), a new technique of designing MAS using or mimicking the human Immune System (IS) behavior for power distribution system restoration and reconfiguration has been pursued. The Immune System is the protector of the human body, formed by millions of cells which together act as a highly intelligent multi-agent system. The designed multi-agent system is capable of detecting and isolating circuit faults and finding a configuration target to restore power. In the search for a configuration, the algorithm takes into account voltage violations, line capacities, and performs load shedding if necessary. This system can and will have the technique requirements for continuous learning through smart sensor based interactions with the system, such as a power distribution system, in order to perform important and necessary procedures as system reconfiguration. 


\section{Deliverable 2. Reports on Economic Evaluation and Economic Feasibility.}

\section{D2.0 Introduction.}

In general, it is anticipated that multiple agent system (MAS) deployment expense can be minimized and economic benefit maximized through implementation strategies that integrate agent functions within various distributed generation (DG), Distributed Energy Resource (DER) and demand side management (DSM) technologies, as well as transmission level assets such as phase sensory and analysis devices, and power electronic switched dynamic reactive support devices [e.g., static volt-ampere reactive (VAR) unit, static VAR compensator (SVC) and static synchronous compensator (STATCOM)].

This affords relatively easy and low cost integration of MAS functionalities and software into the control and communication modules already present for digital signal processor (DSP) controlled DSM and inverter-based DG/DER technologies.

\section{D2.1 Evaluate the Economic Feasibility of Incorporating MAS in CoF.}

The research focus of this task was to develop and refine cost and benefit estimates needed to assess the economic feasibility of implementing the MAS developed by the Advance Power and Electricity Research Center (APERC) at West Virginia University (WVU) into the Circuit of the Future (CoF) developed by Southern California Edison (SCE). These estimates are necessary to assess the potential economic impacts of deploying the agents to facilitate and enhance the efficiency and functionality of an automated electric power circuit.

Studies have shown that the primary driver for implementing automation for distribution feeders is the potential to minimize circuit losses. Studies of feeder and distribution circuits similar in size to the CoF have suggested that savings in the range of $\$ 15,000$ to $\$ 90,000$ per pair of open feeders could be obtained by employing the proposed circuit upgrades. However, the actual savings potential per normal open point depends on factors such as load diversity, time of use, number of sections which could be automated as well as the cost per kWh.

The reduction of outage times through quicker circuit restoration - or at least partial restoration - results in lower energy sales losses and improvements in customer satisfaction levels that may lead to further savings by avoiding potential penalties proposed by regulators for poor customer service. It is estimated that the payback for these investments is in the range of 2 to 5 years. The payback period could be shortened if policy regulators were to institute performance penalties.

\section{D2.2 Assess Social Welfare and Commercial Viability of Deploying MAS in CoF.}

The situation is quite different if societal benefits are factored into the assessment. The benefit-to-cost ratio would then become approximately 1.4, suggesting that implementation would be a worthwhile endeavor. However, the utility would need a mechanism by which it could capture a portion of the societal and consumer benefits. This could be accomplished through rate structures, investment tax credits or some other form of regulatory policy or government incentive. 


\section{D2.3 Develop Case Studies Using Utility Input to Estimate Benefits of MAS in CoF.}

A restoration time of 50 to 90 minutes may be reduced to as little as one minute with MAS automation. Reducing restoration times to this level would translate into a savings of $\$ 2.40$ of customer interruption cost (CIC) per kVA of connected load and would represent an approximate 99\% reduction in restoration time. The following is an initial Case Study for the SCE CoF.

Estimated investment in MAS applied to CoF:

- \$1.1 Million Circuit Hardware Purchases: DG, SVC and Switches.

- \$26,750 Smart Agent Hardware Development.

- \$320,000 Smart Agent Software Development.

- Average Annual Savings for commercial and industrial (C\&I) Customers: \$2.5 million, due to faster restoration times.

- Utility Benefits Revenue from Reduction in Lost Load: \$178,000 assumes loss of entire circuit.

- Agent Communications: Not yet determined.

- Total Cost: \$1.45 million, without communications.

Based upon the average annual outage cost estimates for California C\&I customers, a 99\% reduction in restoration time would provide a cost saving of approximately $\$ 2.5$ million per year. Utilities, on the other hand, would only incur a savings of \$178,000 per year, based on the value of interrupted (unserved) load on the circuit.

\section{D2.4 Document the SCE Support for Performing Economic Feasibility and Benefit Analysis Through CoF Asset Planning.}

Currently, the cost of implementing multiple agents into the CoF with communications is estimated to be a total of $\$ 1.9$ million. This total cost includes the proposed CoF hardware investment by SCE, the costs of the agent hardware and software and the new communications hardware and software. Details of the cost analysis as well as a breakdown of the cost components are provided above.

Based upon the estimated values of the benefits and the costs, the benefit-to-cost ratio for the SCE utility is approximately 0.094, which suggests that such an investment for the utility would not be prudent. However, the avoided cost of a substation upgrade needs to be considered before any definitive conclusions can be drawn regarding the merit of the investment from the utility's perspective.

\section{D2.5 Prepare Topical Report on Economic Analysis and Evaluation of MAS Feasibility.}

The most beneficial function of smart power distribution system automation is automated switching. However, smart switches can be expensive, thus imposing an economic constraint on the degree of circuit automation that can be implemented and placing limits on circuit segmentation. The cost of the switches must be weighed against the benefits associated with fault isolation and the ability to reconfigure the un-faulted circuit sections.

The benefits associated with fast restoration and reconfiguration become important in open market scenarios. Key requirements for such analyses and evaluations are:

- Perform Reliability Analysis:

- Examine Reliability Statistics for Circuit; e.g., the CoF (or Avanti). 
- Examine Reliability Results of Automated Switching Simulation.

- Outline Scenario for Reconfiguration Simulation using Agents.

- Analyze Modifications of MAS focused on Discussion of Optimal Number of Agents.

- Develop Preliminary Cost Estimate for Incorporation of Agents into Circuit.

- Develop Estimates of Consumer and Utility Benefits.

And finally, it is important in order for the Feasibility of MAS Technology to continue its progress in Electric Power Systems, that there be resolve to Perform Future Work, such as:

- Refine the Cost Estimate to incorporate a Multi-Agent System.

- Develop an improved Cost Estimate for System Communications. 


\section{Deliverable 3. \\ Catalog of Existing/Emerging Technologies for Power Systems.}

\section{D3.0 Introduction.}

This Deliverable provides an extended list of existing and emerging technologies that could be integrated into a power system. The list includes those technologies for a next generation distribution system (such as the SCE CoF grid), as well as communication devices and networks for use on future electric power distribution systems and substations.

\section{D3.1 Some Existing/Emerging Technologies.}

Following is a brief discussion of some of the emerging technologies that could be deployed for a next generation power system. Details can be found in the NETL report titled "A Compendium of Smart Grid Technologies.” See website http://www.netl.doe.gov/smartgrid/ referenceshelf/whitepapers/Compendium_of_Technologies.

\section{- Current Limiting Conductor (CLiC).}

The CLiC is a low cost ( $\$ 15$ to $\$ 30 / \mathrm{kVAr}$ ) distributed series impedance device that can remotely operate with preprogrammed logic to inject or remove series impedance and control the flow of power. Since each CLiC is a commoditized module, it can be coupled to the conductor(s) at a transmission or subtransmission tower to balance current flow between circuit phases (or between parallel lines) in order to reduce transients and maximize use of T\&D assets.

\section{- Distributed Series Impedance (DSI).}

The Dynaflo distributed series impedance device is also low cost ( $\$ 20$ to $\$ 40 / \mathrm{kVAr}$ ), and includes similar functionalities as the CLiC. In addition, the Dynaflo DSI can control the flow of power either with wireless or PLC, and can also monitor line conditions like thermal rating, vibrations, icing, etc.

- HTS Synchronous Condenser -- SuperVAR ${ }^{\mathrm{TM}}$ with 2G YBCO HTS wire.

In shunt, High Temperature Superconducting Synchronous Condensers (HTSCC) with second generation (2G) superconducting wire can provide even lower cost voltage support than the $1 \mathrm{G}$ SuperVAR ${ }^{\mathrm{TM}}$. Comparatively small and modular, an HTSCC also can provide distributed, dynamic reactive power compensation with 2 to 4 times peak output for short periods of time without harmonics. In series it can provide dynamic moderate cost power control. The $2 \mathrm{G}$ system is capable of providing higher output at lower cost than a $1 \mathrm{G}$ system since the $2 \mathrm{G}$ system could be all superconducting with a low loss filamentized $2 \mathrm{G}$ wire stator instead of a copper stator. The $2 \mathrm{G}$ SuperVAR ${ }^{\mathrm{TM}}$ has the potential to be $>99 \%$ reliable with only $0.5 \%$ losses.

- HTS Fault Current Limiters (FCL) Using 2G Wire.

FCLs using 2G superconducting wire are being developed that are instantaneous, have 10 times less AC losses, limit 3 to 10 times more current, and have smaller footprints than conventional FCLs. 


\section{- Composite Conductors.}

3M's Aluminum Conductor Composite Reinforced (ACCR) costs from 5 to 8 times more than ACSR conductor but can increase can transmission thermal capacity $150 \%$ to $300 \%$. Composite Technology Corporation's (CTC's) Aluminum Conductor Composite Core (ACCC®) configured also into trapezoidal wire is expected to cost 3 to 5times more than conventional wire, but with a $>100 \%$ capacity increase.

\section{- $1 G$ and 2G HTS Cables.}

HTS cables for medium voltage direct current (MVDC) applications can be used to transmit large quantities of power at reduced voltages (lowering HVDC terminal costs by 25\% to 50\%) and high currents underground or undersea with no losses. Very low heat release allows small trenching requirements. HTS MVDC cables can reduce urban transmission congestion or allow for more intensive urban development.

Cables made with 2G HTS wire can achieve six times higher critical current densities than $1 G$ wire while operating at liquid nitrogen temperatures, and at higher magnetic fields. Lower AC losses have been demonstrated with filamentized $2 \mathrm{G}$ wire. Prices for $2 \mathrm{G}$ wire could be 3 to 10 times cheaper than $1 \mathrm{G}$ wire and have 10 times lower AC losses.

\section{- Advanced OLTC for Transformers.}

Conventional tap changers are designed only to keep the voltage at the low voltage side of the transformer within certain limits around the set point. When the transmission side voltage decreases, traditional on-load tap changers (OLTC) adjust the output and draw more reactive power from the weakened transmission system. An intelligent on-load tap changer uses both high (HV) and low (LV) voltage values and performs an advanced tap changing control.

\section{- Advanced Substation and Distribution Automation.}

Intelligent Electronic Devices (IED) in compliance with IEC 61850, WiFi wireless sensors in the substation and WiMax as the backbone of the system.

\section{- Fault Locator for Distribution Systems.}

Fault location using traveling waves has already been successfully applied in extra-high voltage power grids. However, due to its complexity and high cost, utilities have been reluctant to accept the technique for use in distribution systems. A more cost-effective traveling wave fault location system is being developed for power networks (especially for distribution systems). This new technique uses two traveling wave sensors that capture the current traveling wave flowing from the capacitive equipment to earth and the voltage traveling waves in all three phases of a distribution circuit.

\section{- Advanced Instrument Transformers.}

This measurement system is composed of a current sensor and the associated electronics module that includes an emitter, transmission structure and receiver. The magnetostrictive material of the emitter generates elastic (mechanical) waves under the alternate magnetic field, which is induced by the primary current to be measured. Thus the $50 \mathrm{~Hz}$, or $60 \mathrm{~Hz}$, electrical signals are converted into elastic waves that propagate through a dielectric coupling structure until they reach the receiver, where the elastic waves are converted back into electrical signals. 


\section{- Advanced Signal Distribution.}

Current and voltage measurements from instrument transformers are digitized and then distributed by network systems using either wire or fiber optic (FO) cable. This has the advantage of minimizing noise and interference in the secondary signals, as well as the cost. A fundamental problem with this approach is creating a single digital measurement that is appropriate for all uses.

\section{D3.2 Some Existing Technologies.}

Existing technologies that are related to agent-based control of power distribution systems are discussed in recent academic literature. These articles include:

- Dimeas, “Operation of a Multi-agent System for Microgrid Control.” IEEE Trans on P.S. 2005.

- Feliachi, "Distributed Control Agents Approach to Energy Management in Electric Shipboard Power Systems.” PES GM, 2006.

- Giovanini, "A Primary and Backup Cooperative Protection System Based on Wide Area Agents.” IEEE Trans. on Power Delivery. 2006.

- Ni, "Power System Stability Agents Using Robust Wide Area Control." IEEE Trans. on P.S. 2002.

- Hiyama, "Multi-Agent Based Control and Operation of Distribution System with Dispersed Power Sources.” 2002.

- Liu, "A Mulit-Agent Based Approach for Modeling and Simulation of Bulk Power System Restoration” 2005.

- Nagata, “A Multi-Agent Approach to Power System Restoration.” 2002.

- Nagata, "A Multi-Agent Approach to Distribution System Restoration.” 2004.

- Nagata, “An Agent Approach to Bulk Power System Restoration.” 2005.

- Nordman, "An Agent Concept for Managing Electrical Distribution Networks." IEEE Trans. on Power Delivery." 2005.

- Park and Lim. "Modelling and Control of Agent-Based Power Protection Systems Using Supervisors.” IEEE Proceedings. 2006.

- Sheng, “Agent-Based Self-Healing Protection System.” IEEE Trans. on Power Delivery. 2006. 


\section{Deliverable 4. \\ Report on Challenges for Sensors, Communications and Controls.}

\section{D4.0 Introduction.}

The main challenges for advances in sensors, communications and controls is well illustrated by the new technologies that are needed for smart grid projects, such as the SCE Circuit of the Future.

\section{D4.1 Some Technology Challenges.}

Examples of these technology challenges - fiber optic duct temperature monitoring system, solid state fault current limiter - are illustrated in the schematic of the CoF project shown below.

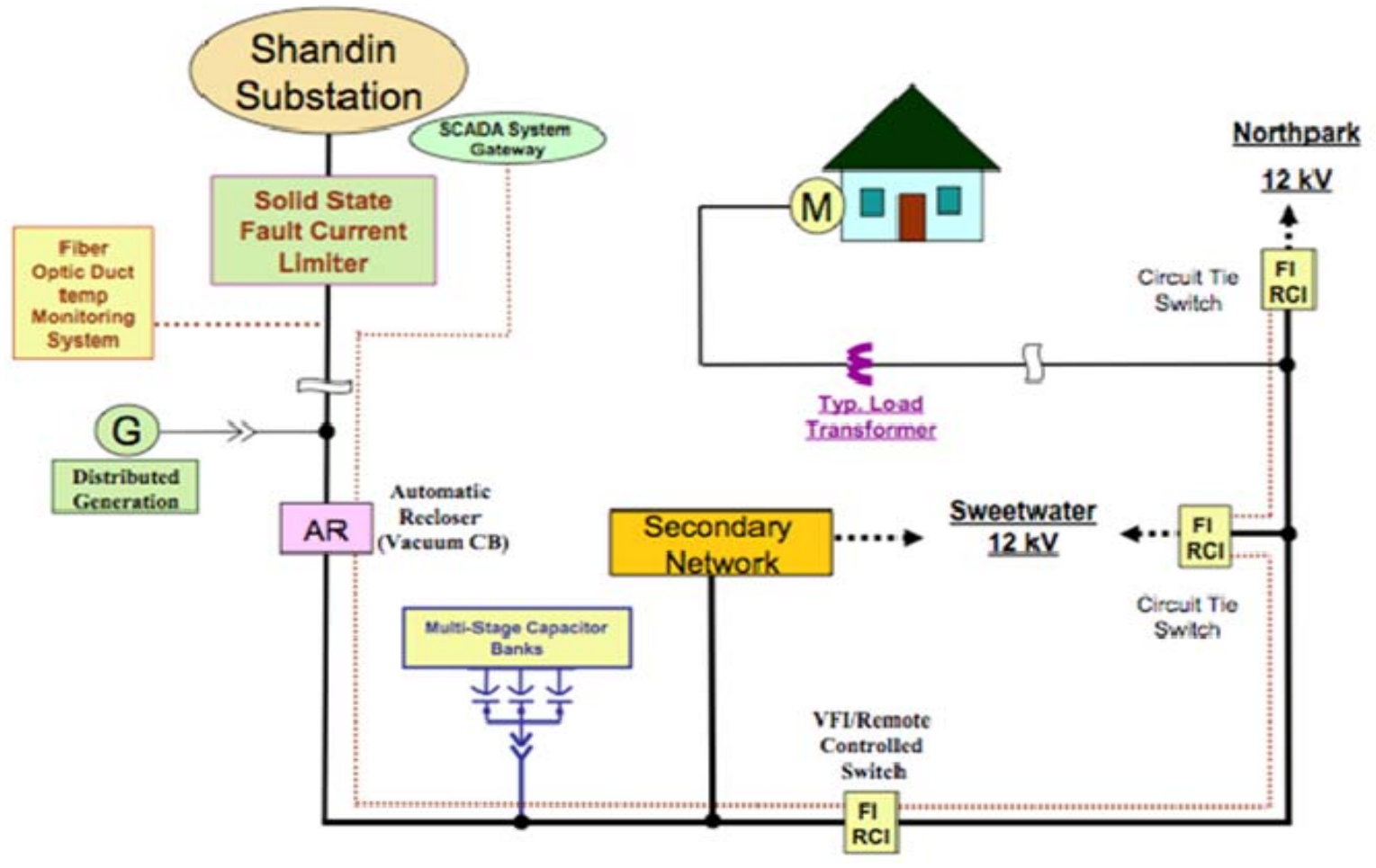

The Final Report for this project contains various references to the identification and the specifications for smart sensors, special communication schemes and newer control methods. 


\section{Deliverable 5. White Paper on Multi-Agent Systems (MAS)}

\section{D5.0 Introduction.}

While there is no single accepted definition of what constitutes an "agent," most would agree that an agent is an object that can sense and act upon its environment with some degree of autonomy. In the context of software-based agents (as opposed to biological ones) the following definition is commonly cited, "An agent is a computer system that is situated in some environment, and that is capable of autonomous action in this environment in order to meet its design objectives.” (Weiss 1999). Agents are capable of flexible autonomous actions through reactivity -- responding in a timely manner, pro-activity -- taking initiative through goal-directed behavior, and social ability-- interacting. A multi-agent system is composed of a population of agents, which interact with each other to reach common objectives, while simultaneously each agent pursues individual objectives (Ferber 1999). Figure D5.1 shows a representation of a software agent, while some of the main characteristics of a software agent are described in Section D5.1.

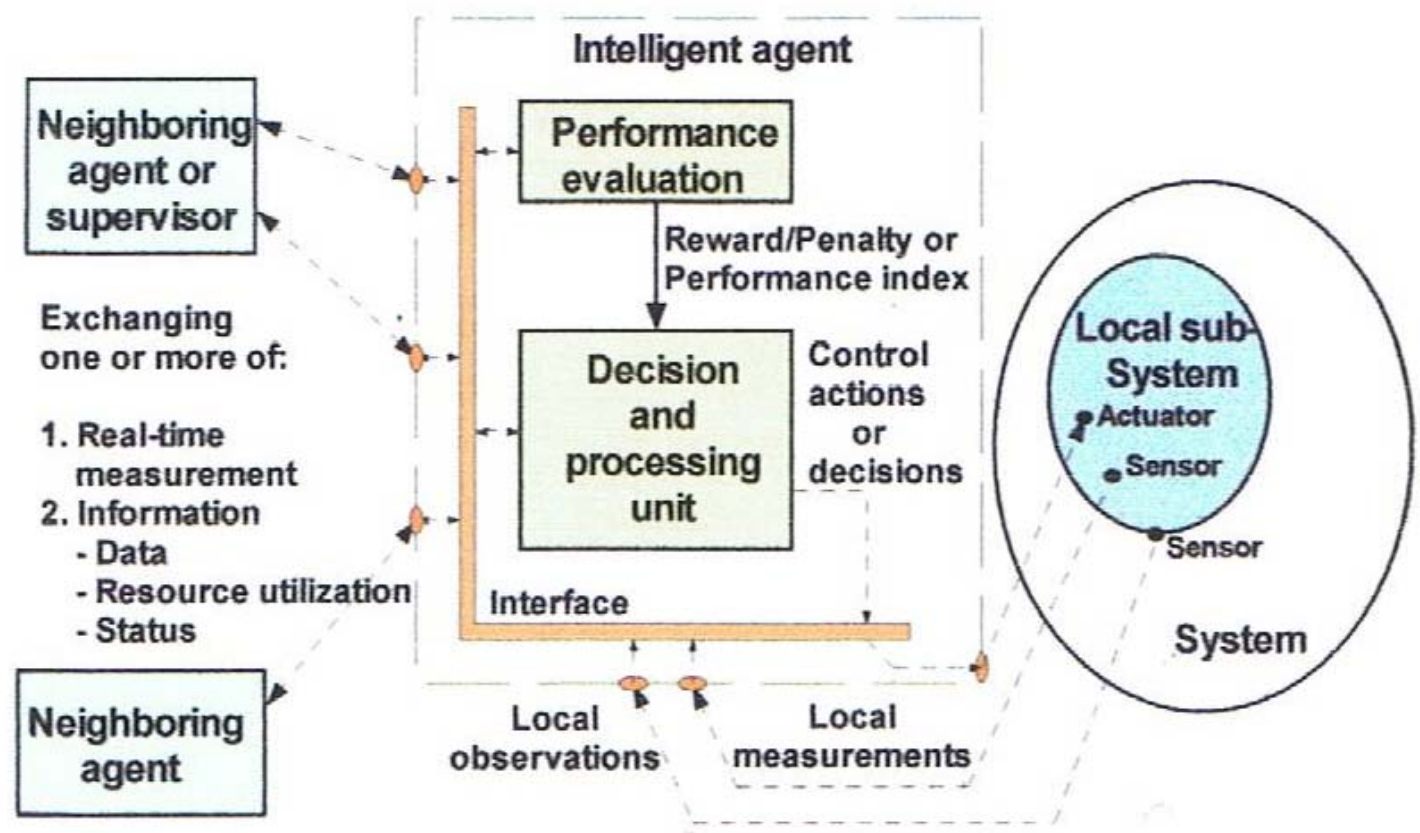

Figure D5.1 A Schematic of an Agent in a Power System.

\section{D5.1 Main Characteristics of a Multi-Agent System.}

Multi-Agent Systems: Agents form loosely-coupled networks of problem solvers and work together to find solutions that are beyond their individual capabilities. Through its distributed cooperative nature and appropriate communication capabilities, the agent based approach 
increases fault-tolerance, avoiding collapse when one or more of its components fail. As an agent can be identified as an entity (e.g., a machine, a plant, a tool or a part), the agent based approach supports incremental design and provides the advantage of scalability. An agent can join a system, start working with other agents, or just leaves a system (without affecting the operation of the system) once it has finished a plan it was engaged in. Because each agent is in close contact with the real world, the system's computational state tracks the state of the world closely. As the overall system behavior emerges from local decisions, the system adjusts itself automatically to changes in the environment or the removal of other agents. Thus a fully functional self-configuring system can be effectively implemented by merely networking agentbased resources.

Environment: An agent's environment consists of the subsystem of the power system that is assigned to a particular agent, and the neighboring agents that communicate with it. Local observations and measurements, and interaction with neighboring agents, constitute the information that is available to the local agent.

Decision and Processing Unit: This constitutes the "brain" of the agent. The agent is expected to act intelligently. Reinforcement Learning and Model Predictive Control are examples of algorithms that could be used by the agents to learn and act on the system. Only a brief overview of reinforcement learning is given below.

Performance Evaluation: To determine whether or not an agent is pursuing an objective satisfactorily an evaluation mechanism is associated with each agent. If the actions of the agent contribute to the overall objective of the system, then the actions of the agents are rewarded, if not they are penalized.

Situation Awareness: Autonomous agents are situated in an environment perceiving signals and acting on the environment. An agent's situational awareness component helps determining operational status, identifying component failures, tracking performance, and analyzing system events that have the potential to degrade system performance and reliability. Most environments will be too complex, so that full state determination through sensing alone will not be feasible. While observer based designs, e.g., Kalman filters, and/or communication of remote information can improve the locally available knowledge, other methods for decision making can be incorporated. For example, decision networks extend probabilistic reasoning of Bayesian networks by both actions under consideration and desirability of the resulting outcomes. Decision networks combine prior and acquired knowledge for efficient decision making under uncertainty and provide explanatory capabilities for actions to be taken.

Termination Detection: Termination detection algorithms are an important part of distributed computing and concurrent programming. The objective of termination detection is to determine the state of all agents in the system and to decide whether all agents are idle. Termination is a global property and difficult to detect by individual agents as their knowledge is limited to local state information and the states of agents connected through direct communication channels. One set of termination detection algorithms requires a unique node to start computations and detect termination. Two examples for this type of algorithm, which are known as diffusing algorithms, are the Dijikstra-Scholten algorithm and credit recovery algorithm. Other algorithms try to take a consistent snapshot of the current computation to determine its status. Examples include the Snapshot algorithms and Chandy-Lamport Algorithm. Another class of algorithms allows any node to start the detection algorithm. Examples are wave based algorithms and graph relabeling algorithms. 


\section{D5.2 Communications for a Multi-Agent System.}

Communication among agents can be achieved by different means. The use of the blackboard architectures, for example, allows global information exchange. Blackboards facilitate information exchange through a common memory location that is known and shared by all agents. Additional measures need to be taken to avoid the possibility of single-point failures due to the inherent centralized nature of the blackboard architecture. Other approaches build on completely decentralized schemes, only exchanging information among neighboring agents.

Steps to standardize the information exchange and support interoperability among agent technologies have been taken and, for example, the Foundation for Intelligent Physical Agents (FIPA), an IEEE Computer Society standards organization, provides specifications for design of agents and agent communication.

=ニニニーニニニニニニーニニニ=

The source of this overview about agents is from Reference [2.17]. 


\section{Deliverable 6. \\ Report on Specific Applications in Modeling and Simulation.}

\section{D6.0 Introduction.}

The Lane Department of Computer Science and Electrical Engineering at West Virginia University maintains an analog power simulator. The simulator is a low power hardware replica of a distribution system that contains commercial, industrial and residential loads. Power can be supplied to the loads from different internal and external circuits and generators and routed in a variety of ways.

\section{D6.1 Development of a Micro-Grid Simulator.}

As part of this project, the simulator was retrofitted to represent a micro-grid of the future by installing digital relays, intelligent electronic devices, distributed energy resources (generation and storage), and a FACTS device, as shown in the diagram of Figure D6.1.

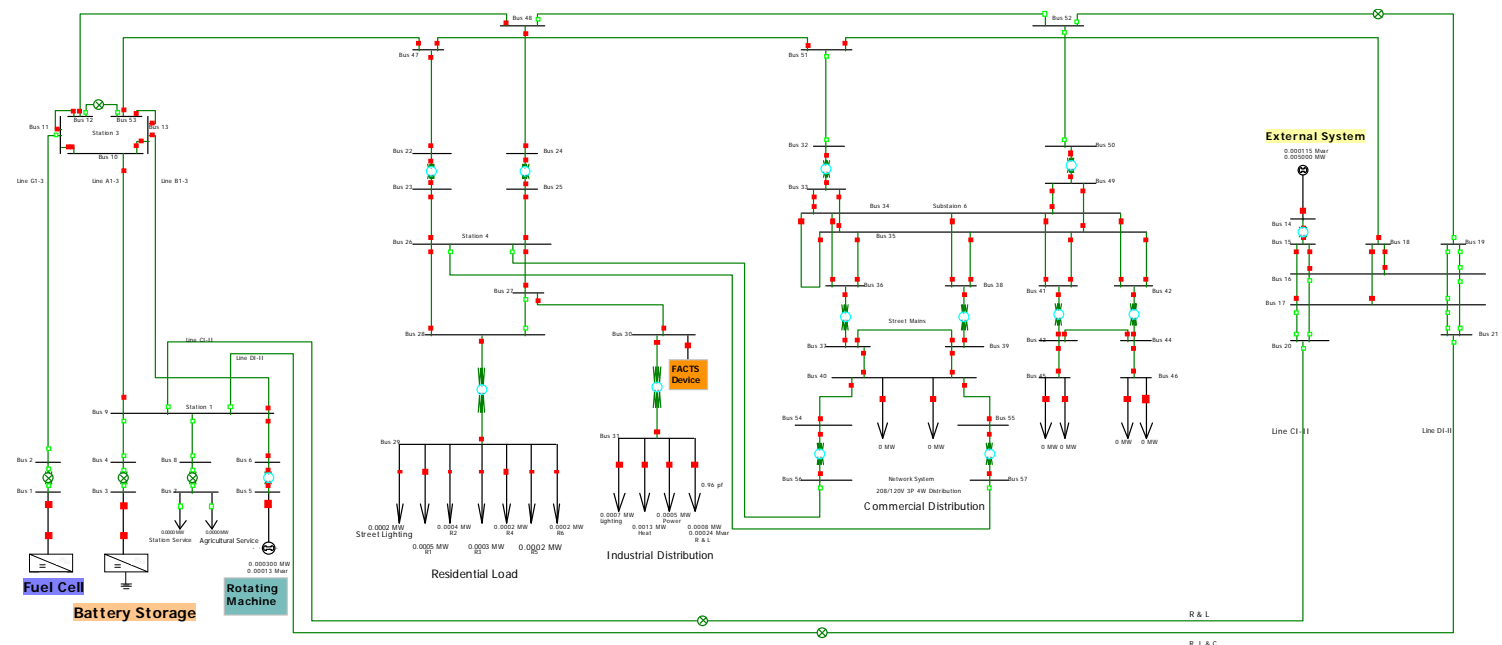

Figure D6.1. Diagram of Power Simulator with Fuel Cell, Battery Storage, and FACTS Device.

This new digital hardware was integrated with the older electromechanical hardware commonly found in real world power systems. This specific endeavor included an effort to update an analog power system in order to model and simulate the modern power system. The retrofit would also allow the simulator to be used for future research, such as autonomous reconfiguration schemes for power distribution circuits. 


\section{Deliverable 7. \\ Reports on Simulation Studies and Laboratory Tests of a Multi-Agent System.}

\section{D7.0 Introduction.}

Included below are two reports on simulation studies based on multi-agent systems, and one report on a laboratory effort to test multi-agent systems.

\section{D7.1 A Multi-Agent Coordinated Strategy.}

The research involves the detection and elimination of voltage violations in a power system using multi-agent technology. With efforts being made to keep power system voltage within prescribed limits, multi-agent systems with their pro-active behavior and communication capabilities could play a vital role in keeping the voltages within the specified limits.

The objective of this research was to develop a multi agent coordinated strategy, wherein, under a specified threshold limit for the bus voltages, the Bus Agents (BA) and the SVC Agents (SA), used in this scheme, detect the violation and hence change the SVC settings to improve voltages on the bus by injecting more reactive power in the system.

Today, FACTS devices are considered to be very useful in controlling the voltage and power flow in a power system. Each FACTS device is assigned a primary zone of control and a primary objective, which may be to control some disturbance, or to improve the stability of the power system. The influences of FACTS devices are not restricted to one bus or line. Since changing the voltage at a certain bus also changes the voltage at the other buses in the grid, coordination of FACTS devices is very necessary. Another function of FACTS devices is that they can support adjacent devices in the network, to the extent permitted by the specifications of each device. To gain the maximum advantage, FACTS devices have to be effectively coordinated with other devices, loads, and other generation sources. Failure to do so may adversely affect the dynamic stability of the power system. So for both steady state and dynamic operation of power systems, controlling and coordinating FACTS devices becomes essential.

\section{D7.2 Spectral Clustering Techniques for Power System Partitioning.}

A spectral clustering algorithm is used to logically partition the power network into clusters of connected buses. In the algorithm, a clustering parameter is selected that represents the notion of electric distances. To tune the clustering algorithm for partitioning a power system and to give importance to electrical properties of the system, the bus impedance matrix is used to acquire the necessary information for clustering.

Agents are then assigned to each cluster or partition. This modified approach scales well as the size of the power system increases by reducing both the total number of agents in the system and the communication overhead. However, to gain these benefits, the agents in the clusters must acquire additional knowledge about the topology in their cluster.

Also, a scalable multi-agent architecture using fewer agents is described. A cluster agent is assigned to each cluster or partition instead of assigning an agent to each bus (as in the decentralized topology-independent MAS). This results in a smaller agent system in which cluster agents can communicate with each other and work in coordination to perform different functions. By dividing the large system into logical clusters and assigning these to different 
agents, the size and complexity of the problem are reduced for each agent, thereby making the load flow calculations, monitoring, reconfiguration, or restoration efficient and manageable. A switch agent turns a reconfiguration scheme on or off based on the chosen path and given constraints.

\section{D7.3 Effort to Demonstrate Multi-Agent Concepts on Laboratory-scale Power System Simulator.}

Another activity during this project involved the retrofit of a Laboratory Simulator at WVU to mimic a future micro-grid. A three-stage effort was undertaken to install digital relays, intelligent electronic devices and control agents, distributed energy resources (generation and storage), and a FACTS device.

\section{- Stage One - Addition of Digital Relays}

The first step in the upgrade of the WVU simulator was to install new digital relays contributed by Schweitzer Engineering Laboratories, Inc. (SEL). Following is a list of equipment donated by SEL.
4 SEL 751A Feeder Protection Relays
1 SEL 387A Current Differential Relay
1 SEL 300G Generator Relay
4 SEL 351 Over-Current Protection Relays
1 SEL 351S Protection Relay
1 SEL 734 Revenue Metering System
1 SEL 3351 System Computing Platform
"Subnet" Software
All necessary cables

Some of the donated digital relays were installed next to their complementary analog counterparts and wired in parallel with the existing relays to allow either to be used for teaching or research purposes. Figure D7.1 shows the simulator with the newly installed equipment.

Stage one is almost complete, the new relays still need to be wired up and their electromechanical contactors installed in parallel with those of their analog counterparts. Some of the relays were installed next to their complementary analog counter parts and have been wired in parallel with the existing relays which allows either to be used for teaching or research purposes. These new relays still need to be wired in parallel with those of their analog counterparts.

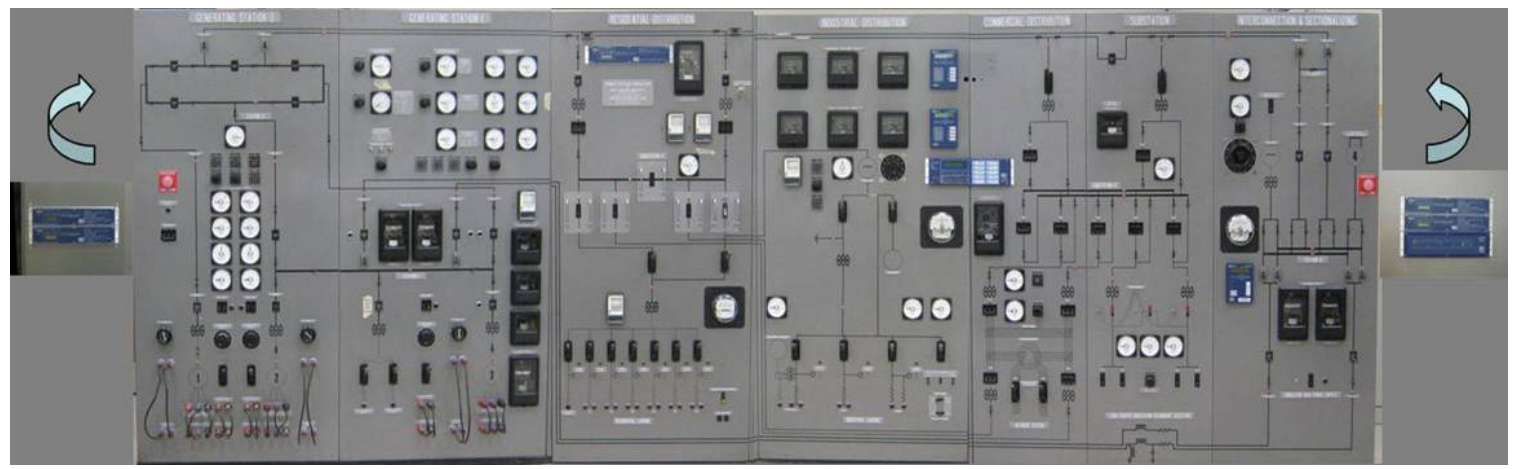

Figure D7.1 Photograph of Laboratory Simulator with new SEL Equipment Installed. 
In order to test different control algorithms, agent based intelligent switches will be installed in parallel with the existing manual switches. As delivered, the simulator had approximately 100 manually operated switches that allow the system to be reconfigured in a enormous number of ways in order to reroute power between generation and loads. In stage two of this effort, the majority of those switches will replicated by microcontrollers that will allow communication between switches as well as intelligence at each of these switches. When not needed, the MCU controlled switches can be turned off and the simulator used in its original configuration.

A search was made to find a compact microcontroller that gave as much processing and communication capability as possible to allow the algorithm developers as large a canvas as possible to try their ideas on. A demo board from Freescale the M52259DEMOKIT was selected. It has a 32 bit $80 \mathrm{MHZ}$ processor with 512Kbytes of flash memory, 64Kbytes SRAM, and a cryptographic accelerator unit for secure communications without taxing the main processor. It comes with two USB ports, one 10/100 Ethernet port, an RS-232 port and a high speed CAN bus port, all in a 3”x3.5” two board package for \$49. Figure D7.2 shows the demo board with its communication ports.

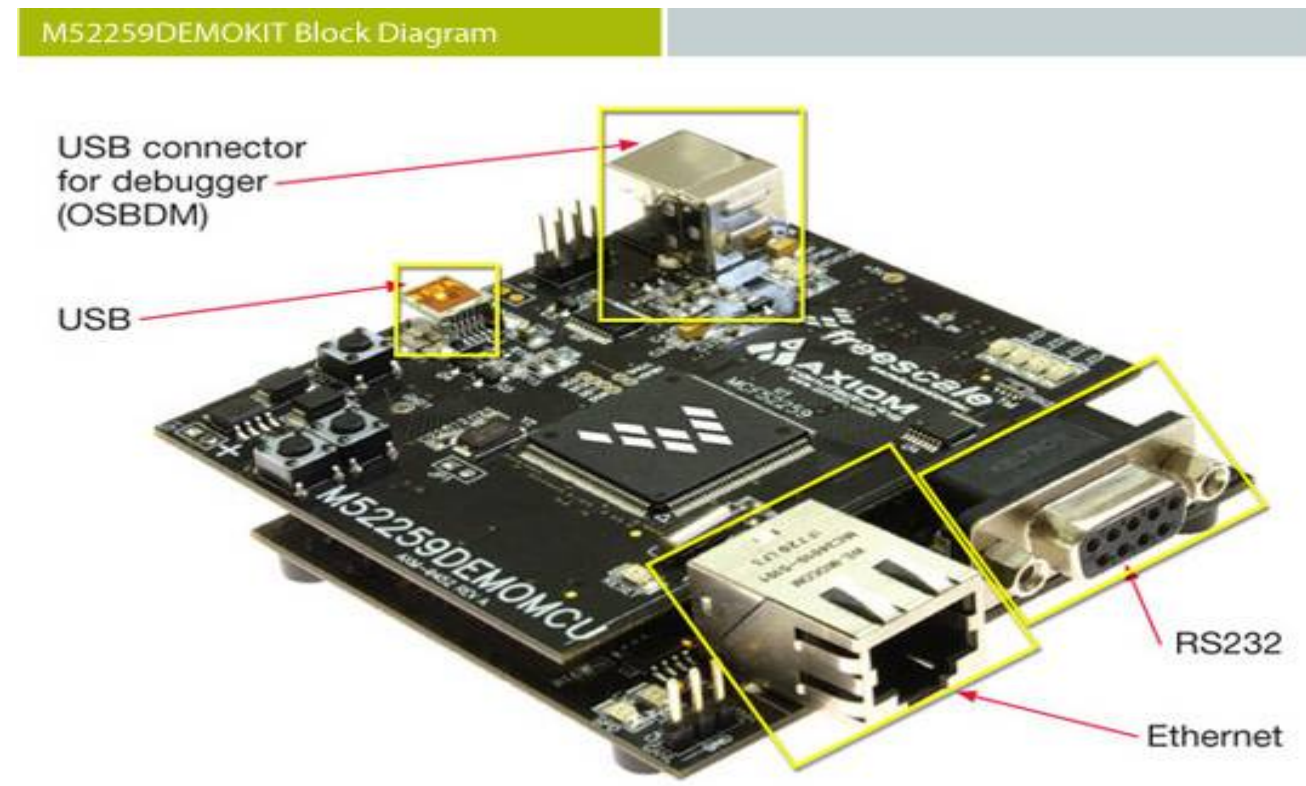

Figure D7.2. Freescale M52259DEMOKIT

Given the power consumption, size, and noise of approximately one hundred 9 amp contactors, the design of some solid state switches are being investigated that can handle that amperage and be controlled by the MCU via an opto-isolator. Off the shelf solid state contactors are cost prohibitive at about $\$ 80$ a piece. Using relatively common parts, it is thought that a three phase solid state switch can be put together for about \$10 in the numbers required for this project 


\section{Main Project Accomplishments}

\section{Critical Fault Protection for the CoF has been Improved:}

- Improved fault detection using multi-agent systems was demonstrated through simulation studies. The multi-agent models were simulated in the Java Agent Development Framework and MATLAB.

\section{Fast, Safe Reconfiguration of the CoF can be Achieved.}

- Using multi-agent systems, fast safe reconfiguration of the CoF was demonstrated through simulation in the Java Agent Development Framework and MATLAB.

- A better model of the CoF was developed using new techniques and algorithms.

0 A reconfiguration algorithm was developed based on a self-stabilizing maximum flow algorithm that uses nodes to represent software agents. This algorithm consists of guarded statements to modify node and link variables such as flow, priority, residual capacity, supplied demands and unsupplied demands. Each agent executes these guarded statements asynchronously, and informs neighboring agents of any changes that have had occurred. An agent is idle if it does not have any statement to execute, and the algorithm converges when all agents are idle.

o A termination-detection algorithm was developed to service the reconfiguration algorithm. The termination-detection algorithm is based on the wave algorithm concept, in which the wave computation allows continuous detection and responds to the environmental changes of the reconfiguration algorithm. An agent becomes idle when it has no basic computation to perform and forward certain kinds of waves. Any agent receives certain type of waves detects global termination of the reconfiguration algorithm.

o A graph model was developed for the CoF using rooted spanning trees to represent the radial power supply system. In this model, a node represents either a power source (the rooted node or nodes), or a load, while edges represent the physical power line connections of the system. Other edges in the graph model, but not in the spanning tree, represent the switches. The feeders in the power system are represented by rooted and directed subtrees.

o A multi-agent system was developed (for the reconfiguration for the $\mathrm{CoF}$ ) that performs fault detection in a decentralized manner and the fault reconfiguration detection in a centralized manner by utilizing the graph model. In this system, a multi-agent architecture consisting of global, local load and local switch agents is deployed in the graph model. Local load agents and local switch agents are also represented by the corresponding nodes and edges. They have the capability of communicating locally (limited to the neighboring agents). These agents have local information pertaining to that particular region only. The global agent operates in a centralized manner.

- A reconfiguration strategy was developed by using mathematical programming, and making better application of deploying multi-agent technology with the graph model. During the reconfiguration process, system conditions are modeled as constraints in the mathematical programming, whose goal is to maximize the electrical current to the loads in the system with available power supply. 


\section{Operational Deployment of the CoF is Better Appreciated.}

- An incremental cost estimate for upgrading the CoF and employing the MAS was developed.

o An overall cost estimate was constructed for the CoF based on incremental modifications made to automate the circuit and employ the agents. Estimates were developed for individual cost elements which includes installation costs. Because the analysis was based on a circuit upgrade, all costs are assumed to be incremental and do not include any operating and maintenance (O\&M) or capital costs that would have otherwise been incurred.

- An estimate of the potential benefits from a restoration and reconfiguration perspective was developed.

o The potential benefits associated with the modernization of the $\mathrm{CoF}$ were identified and attempts were made to assign quantitative values to them. The resulting list includes both direct benefits and indirect benefits. The departure of key SCE researchers assigned to the project, and SCE's withdrawal after their departure, prevented the calculation of the actual total benefits. Therefore the benefits estimate relied on general inferences and results of similar studies to augment the actual benefit calculations made from available data. A list of potential benefits and the associated beneficiaries is provided as part of Task 4. Benefits are calculated and classified by beneficiary.

o The costs that were displaced or deferred because of the circuit modification were included as a cost avoidance benefit attributed to the $\mathrm{CoF}$.

- The potential economics of the above modifications were developed through the use of a cost benefit analysis.

o The economic feasibility appears to be only marginal from the operational perspective given the uncertainty inherent in the cost and benefit estimates.

o The results improve significantly when the upgrade is analyzed from a boarder socioeconomic perspective. 


\section{Appendices}

\section{Appendix 1. \\ Project Fact Sheet}

\section{Integrated Control of Next Generation Power Systems}

\section{Background}

The generation, transmission and distribution of electrical power nationally continues to experience increased load demand, added environmental constraints and revised market strategies. In addition, these electrical power systems are operated by utility companies who have been reluctant to undertake the significant capital investments needed to enhance the reliability of the existing power systems. Thus, our national electrical power system has become overstressed and is subject to potential failures. Technological advances need to be developed that utilize advances in communications and controls that enable automatic detection of power system problems and automatic reconfiguration of the electrical transmission and distribution grid to minimize potential impacts of any natural and/or human caused disruptions.

\section{Goals}

The main goal of this project focused on enhancing the reliability, security and economics of our national electrical power system by pursuing two key objectives:

o Advance the technological awareness of integrating multi-agent controls and associated hardware (such as sensors and distributed resources) into an existing electrical power system, and

o Assess the feasibility of multi-agent controls in effectively operating and controlling a next generation power system (such as the Circuit of the Future developed by Southern California Edison).

\section{How Does It Work?}

Control agents will be used to form a coordinated multi-agent system (MAS). Through sensors and communications a MAS, when integrated into an existing electrical power system, will monitor the operating conditions and autonomously reconfigure the transmission and distribution grid to manage the flow of electricity and assure delivery of critical power. Such integration of a MAS within an existing power system will enable our national electrical infrastructure to be more resilient to natural and other events that could otherwise result in blackouts.

\section{What are the Benefits?}

Successful completion of this applied research project will advance both the fundamental understanding and the technical development and validation of MAS as an intelligent system control device for existing power systems. In addition, related system devices and autonomous operating strategies will be functionally developed and at least conceptually integrated into an existing power system. Ultimately, the advances in smart agent control and intelligent hardware devices achieved during the project will yield insights about the feasibility of such new technologies toward defining a more interoperable grid for next generation power systems. 


\section{What are the Primary Applications?}

The primary application for the proposed MAS architecture is as an autonomous management scheme for an existing or next generation electric power system. The layered architecture design is shown in Task 3. This scheme can be readily integrated into an electrical transmission and distribution grid and addresses the operational issues of dynamic faults, operating contingencies and system diagnostics. The proposed MAS design is suitable for an existing or evolving electric power system, and will enable an autonomous, resilient and selfhealing next generation power system.

\section{What is the Status of MAS and Accomplishments to date?}

During this project, smart-agent based energy management scheme (originally developed to control the electric power system on a ship) was transitioned to better manage the electric power system for a utility company. Part of the effort included cataloguing relevant existing advanced control technologies and communication protocols that would guide the direction of this project toward technical developments that are commercially viable.

\section{What is the Market Potential?}

The smart agent control technology developed in this project has a promising market potential in the electric power industry, as well as other industries. Several electric utility companies are already pursuing integration of MAS-based control into their existing power systems. During this project business cases were developed to assess the basic economic benefits achievable through application of the extended enhancements of the developed technologies to an existing as well as a next generation power system. These cases range from simple deployment of the MAS control technology in an existing power system, to maximizing the MAS control technology in a next generation power system by considering various strategies

that involve extensions such as phasors, sensors, reactive support devices and distributed resources. 


\section{Appendix 2. \\ Project Publications}

\section{A2.1 Journal Publications, Conference Proceedings, Symposium Papers and Meeting Presentations and Posters}

[1] P. Hines, S. Hamilton, R. Yinger, C. Vartanian, A. Feliachi, and K. Schoder, "Integrated, Agent-Based, Real-time Control Systems for Transmission and Distribution Networks," Proceedings of the GridWise Architecture Council, Grid Interop Forum, Albuquerque, NM, November 7-9, 2007.

[2] K. Schoder, A. Feliachi, P. Tulpule and H-J. Lai, "Distributed Approaches for Determination of Reconfiguration Algorithm Termination," Automation Controls Symposium, Biloxi, MS, December 10-11, 2007.

[3] A. Karimi and A. Feliachi, "Decentralized Adaptive Backstepping Control of Electric Power Systems,” Electric Power Systems Research Journal, Vol. 78, Issue 3, pp. 484-493, March 2008.

[4] A. Al-Hinai and A. Feliachi, "Microturbines Load Following Controller Design in Deregulated Power Distribution Systems," IEEE PES General Meeting, Pittsburgh, PA, July 2008.

[5] H. Wan, K.P. Wong and C.Y. Chung, "Multi-agent Application in Protection Coordination of Power System with Distributed Generations," IEEE PES General Meeting, Pittsburgh, PA, July 2008.

[6] C. Vartanian, D. Law, P. Hines, R. Yinger, S. Hamilton and A. Feliachi, "Agent-based Distribution Circuit Automation," IEEE PES General Meeting, Pittsburgh, PA, July 2008.

[7] R. Belkacemi and A. Feliachi, " An Immune System for Power System Automation and Self Healing," Student Poster, IEEE PES General Meeting, Pittsburgh, PA, July 2008.

[8] S. Eftekharnejad,, A. Feliachi (Advisor), "Reinforcement Learning Based Agent Approach to Load Frequency Control in Electric Power Systems," Student Poster, IEEE PES General Meeting, Pittsburgh, PA, July 2008.

[9] A. Feliachi, "Integrated Control of Next Generation Power Systems," Award No. DEFC2606NT42793, US DoE RDIS Peer Review Meeting, October 2008.

[10] R. Belkacemi and A. Feliachi, "Design and Deployment of a Multi-Agent System on a Hardware Prototype”

[11] S. Chouhan, H. Wan, H-J. Lai, A. Feliachi and M. A. Choudhry, "Intelligent Reconfiguration of Smart Distribution Network using Multi-Agent Technology" IEEE PES General Meeting 2009. 
[12] K. Nareshkumar, M. A. Choudhry, H.J. Lai and A. Feliachi, "Application of Multi-Agents to Fault Detection and Power Distribution Systems Reconfiguration,” IEEE PES General Meeting 2009.

[13] R. Belkacemi and A. Feliachi, "Multi-Agent Design for Power Distribution System Reconfiguration Based on the Artificial Immune System Algorithm."

[14] R. Belkacemi and A. Feliachi, "A Decentralized Immune Based Automatic Generation Control Design in Compliance with NERC’s Standards,” PSEC Meeting 2009.

[15] A. Karimi, M. A. Choudhry and A. Feliachi, "Coordinated Backstepping Controls for Power Systgem Stability Enhancement,” NAPS Meeting 2008.

[16] A. Hajizadeh, M. A. Golkar and A. Feliachi, "Control of Hybrid Fuel Cell/Energy Storage Power Conversion System under Unbalanced Voltage Sag Conditions,” IEEE EC Meeting 2009.

[17] A. Al-Hinai and A. Feliachi, "Multi-Agent Based Controllers for Distributed Generators," IEEE GCC Meeting 2008.

[18] A. Karimi, S. Eftekharnejad and A. Feliachi, "Reinforcement Learning Based Backstepping Control of Power System Oscillations," Electric Power Systems Research Journal, Vol. 79, pp. 1511-1520, 2009.

\section{A2.2 Dissertations and Theses.}

[1] B. McGee, "Multi-Modal Communication," MSEE Thesis, Lane Department of Computer Science and Electrical Engineering, West Virginia University, May 2008.

[2] A. Karimi, "Power System Damping Controllers Design using a Backstepping Control Technique," PhD Dissertation, Lane Department of Computer Science and Electrical Engineering, West Virginia University, August 2008.

[3] K. Nreshkumar, "Application of Multi-Agents to Power Distribution Systems," MSEE Thesis, Lane Department of Computer Science and Electrical Engineering, West Virginia University, August 2008.

[4] S. Eftekharnejad, "Reinforcement Learning-Based Control Design for Load Frequency Control,” MSEE Thesis, Lane Department of Computer Science and Electrical Engineering, West Virginia University, August 2008.

[5] S. Chouhan, "Agent-Based Modeling of Power Distribution Systems," MSEE Thesis, Lane Department of Computer Science and Electrical Engineering, West Virginia University, February 2009. 
[6] A. Vedam, "Design and Analysis of a Hybrid Guideway Heating System for Morgantown Personal Rapid Transit,” MSME Thesis, Department of Mechanical and Aerospace Engineering, West Virginia University, May 2009.

\section{A2.3 APERC Documents.}

[1] K. Schoder and P. Hines, "Improving and Evaluating Reliability of Electric Distribution Systems through Automated Switching Schemes,” APERC Document, November 27, 2008.

[2] P. Hines, "Review of Existing Technologies and Technical Performance Requirements," APERC Document, March 5, 2009.

[3] S. Wan, “Evaluate Performance Requirements to Access a MAS System, etc," APERC Document, March 27, 2009.

[4] S. Wan, "Multi-Agent Based Architecture for Protection and Coordination" APERC Document, October 30, 2009. 


\section{Appendix 3. Bibliography \& Cited References}

\section{A3.1 Task 1}

[1.1] Dimeas, “Operation of a Multi-agent System for Microgrid Control.” IEEE Trans on P.S. 2005.

[1.2] Feliachi, "Distributed Control Agents Approach to Energy Management in Electric Shipboard Power Systems.” PES GM, 2006.

[1.3] Giovanini, “A Primary and Backup Cooperative Protection System Based on Wide Area Agents.” IEEE Trans. on Power Delivery. 2006.

[1.4] Ni, "Power System Stability Agents Using Robust Wide Area Control.” IEEE Trans. on P.S. 2002.

[1.5] Hiyama, "Multi-Agent Based Control and Operation of Distribution System with Dispersed Power Sources.” 2002.

[1.6] Liu, “A Mulit-Agent Based Approach for Modeling and Simulation of Bulk Power System Restoration” 2005.

[1.7] Nagata, “A Multi-Agent Approach to Power System Restoration.” 2002.

[1.8] Nagata, “A Multi-Agent Approach to Distribution System Restoration.” 2004.

[1.9] Nagata, “An Agent Approach to Bulk Power System Restoration.” 2005.

[1.10] Nordman, “An Agent Concept for Managing Electrical Distribution Networks.” IEEE Trans. on Power Delivery.” 2005.

[1.11] Park and Lim. "Modelling and Control of Agent-Based Power Protection Systems Using Supervisors.” IEE Proceedings. 2006.

[1.12] Sheng, “Agent-Based Self-Healing Protection System.” IEEE Trans. on Power Delivery. 2006.

[1.13] R. Neal, "Distribution Circuit of the Future at Southern California Edison.” Proc. IEEE PES Power Systems Conference and Exposition, 2004.

\section{A3.2 Task 2}

[2.1] A. Feliachi, Project Fact Sheet for "Integrated Control of Next Generation Power Systems" Project, US DoE Office of Electricity Delivery and Energy Reliability, 2006.

[2.2] R. Neal, "Distribution Circuit of the Future at Southern California Edison.” Proc. IEEE PES Power Systems Conference and Exposition, 2004.

[2.3] M. R. Tousi , S. H. Hosseinian and A.H. Menhaj, "Multi-Agent Based Voltage Control of STATCOMs to Enhance Elimination of Voltage Disturbances in Power System Contingencies”, Amirkabir University of Technology, Tehran, Iran 
[2.4] J. M. Solanki and N. N. Schulz, "Using Intelligent Multi System for Shipboard Power Systems Reconfiguration”, Proceedings of the $13^{\text {th }}$ International Intelligent Systems Application, Nov. 2005.

[2.5] H. F. Wang, "Multi-agent co-ordination for the secondary voltage control in power system contingencies," pp. 61-66, 2001.

[2.6] S. Gehao, J. Xiuceng, and Z. Yi Coordination For Multi-Agent Based Secondary Voltage Control in Power System," IEEE/PES Transmission and Distribution Conference \& Exhibition: Asia and Pacific, Dalian, China, 2005.

[2.7] InterPSS Power System Simulation Project, see: http://www.interpss.org

[2.8] JADE - Java Agent DEvelopment Framework, see: http://jade.tilab.com.

[2.9] K. L. Butler-Purry and N. D. R. Sarma, "Self-Healing Reconfiguration for Restoration of Naval Shipboard Power Systems,” IEEE Transactions on Power Systems, 19 (2): 754762, May 2004.

[2.10] K. L. Butler-Purry, N. D. R. Sarma and V. R. Prasad, "Network Reconfiguration for Service Restoration in Shipboard Power Distribution Systems," IEEE Transactions on Power Systems, 16:653-661, November 2001.

[2.11] S. B. Ganesh, K. Schoder, K., H.-J. Lai, A. Al-Hinai and A. Feliachi, "Energy Management System with Automatic Reconfiguration for Eletric Shipboard Power Systems"

[2.12] A. Feliachi, K. Schoder, S. Ganesh and H.J. Lai, "Distributed Control Agents Approach to Energy Management in Electric Shipboard Power Systems,” Proc 2006 IEEE PES General Meeting, Montreal, Canada, June 2006.

[2.13] K. Schoder, A. Feliachi, P. Tulpule and H-J. Lai, "Distributed Approaches for Determination of Reconfiguration Algorithm Termination," Automation Controls Symposium, Biloxi, MS, December 10-11, 2007.

[2.14] S. Chouhan, H. Wan, H.J. Lai, A. Feliachi and M. A. Choudhry, "Intelligent Reconfiguration of Smart Distribution Network using Multi-Agent Technology” IEEE PES General Meeting 2009.

[2.15] K. Nareshkumar, M. A. Choudhry, H.J. Lai and A. Feliachi, "Application of MultiAgents to Fault Detection and Power Distribution Systems Reconfiguration” IEEE PES General Meeting 2009.

[2.16] R. Belkacemi and A. Feliachi, "Multi-Agent Design for Power Distribution System Reconfiguration Based on the Artficial Immune System Algorithm" proceedings, ISCAS 2010, Paris, France, May 30-June 2, 2010.

[2.17] A. Feliachi, K. Schroder, S. Eftekharnejad and A. Karimi, "Agents: Definition, Processing and Decision Making, Situation Awareness,", Book: Agent Based Coordination of Power System Controllers, pp 8-12. 


\section{A3.3 Task 3}

[3.1] M. R. Tousi , S. H. Hosseinian and A.H. Menhaj, "Multi-Agent Based Voltage Control of STATCOMs to Enhance Elimination of Voltage Disturbances in Power System Contingencies”, Amirkabir University of Technology, Tehran, Iran.

[3.2] J. M. Solanki and N. N. Schulz, "Using Intelligent Multi System for Shipboard Power Systems Reconfiguration," Proceedings of the $13^{\text {th }}$ International Intelligent Systems Application, Nov. 2005.

[3.3] H. F. Wang, "Multi-agent co-ordination for the secondary voltage control in power system contingencies,” pp. 61-66, 2001.

[3.4] Sheng Gehao, Jiang Xiuceng and Zeng Yi "Coordination for Multi-Agent Based Secondary Voltage Control in Power System,” IEEE/PES Transmission and Distribution Conference \& Exhibition: Asia and Pacific, Dalian, China, 2005.

[3.5] InterPSS Power System Simulation Project. See http://www.interpss.org.

[3.6] JADE - Java Agent DEvelopment Framework. See http://jade.tilab.com.

\section{A3.4 Task 4}

[4.1] Walton, C.M. and Friel, R., "Benefits of Large Scale Urban Distribution Network Automation and their Role in Meeting Enhanced Customer Expectation Regulator Regimes”, CIRED 2000.

[4.2] Cepedes, R., Mesa, L., and Schierenbeck, A., "Distriburion Management System at Epressas Publicas de Medellin (Columbia)”, CIRED 2000.

[4.3] Chen, R.L., Sabir, S., "The Benefits of Implementing Automation and Systems Monitoring in the Open Electricity Market”. Proceedings Canadian Conference on Electrical and Computer Engineering, Toronto, 2001.

[4.4] http://www.netl.doe.gov/energy-analyses/pubs/WV SGIP_Final Report rev1 complete.pdf.

[4.5] The Cost of Power Disturbances to Industrial and Digital Economy Companies, EPRI, Palo Alto, CA, p20, 2001. 